\title{
Simulations for an experiment to probe the in-medium properties of photoproduced vector mesons
}

\author{
by \\ Clarisse Tur \\ Licence de Physique Fondamentale \\ Université de Paris Sud, Orsay, 1999 \\ Maitrise de Physique Fondamentale \\ Université de Paris Sud, Orsay, 2000 \\ Submitted in Partial Fulfillment of the \\ Requirements for the Degree of Master of Science in the \\ Department of Physics and Astronomy \\ University of South Carolina
}

2003

Department of Physics and Astronomy

Department of Physics and Astronomy

Director of Thesis 2nd Reader

Dean of The Graduate School 


\section{CONTENTS}

Page

LIST OF TABLES ........................... iv

LIST OF FIGURES ......................... vi

Chapter

I. Introduction ..................... 1

1.1 What is g7 about? .................. 2

1.2 Change of the properties of the vector mesons inside nuclear matter: a historical overview ......... . 3

1.3 Why a photon induced reaction? . . . . . . . . . 9 9

1.4 Why the rho meson production? . . . . . . . . 10

1.5 Why the leptonic decay channel? . . . . . . . . . . 11

1.6 The production mechanism .............. 11

II. The experimental setup . . . . . . . . . . . . . 14

2.1 CEBAF: The continuous electron beam accelerator facility . . 16

2.2 The Hall-B equipment . . . . . . . . . . . . . 21

2.2.1 The production and tagging of the photon beam . . . 22

2.2.2 The CLAS detector . . . . . . . . . . 28

2.2.3 The photon beam flux ........... 50

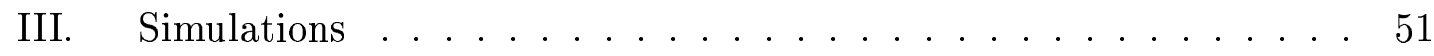

3.1 The g7 target ....................... 52 
3.2 CLAS resolution for vertex reconstruction $\ldots \ldots \ldots \ldots$

3.3 Acceptance/Systematics . . . . . . . . . . . . . 57

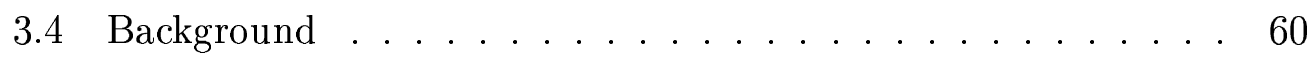

3.4.1 The nature of the background . . . . . . . . 61

3.4 .2 The background simulations . . . . . . . . . 63

3.5 Other issues . . . . . . . . . . . . 75

3.5.1 The angular distribution of the outgoing lepton pair . . 75

3.5.2 The events that give 2 hits in the same sector of CLAS 77

3.5.3 The electron/pion discrimination method . . . . . . 79

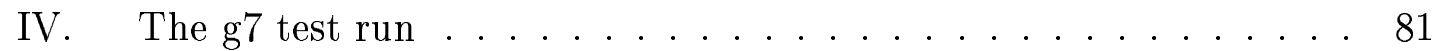

V. Summary and conclusion . . . . . . . . . . . 83

VI. Appendix A: The simulation tools . . . . . . . . . . 85

VII. REFERENCES . . . . . . . . . . . . . . . . 87 


\section{LIST OF TABLES}

1.1 Characteristics of the $\rho, \omega$ and $\phi$ mesons . . . . . . . . . 10

2.1 Main machine parameters for CEBAF . . . . . . . . . 20

2.2 Summary of the CLAS design goals . . . . . . . . . . . . . 29

3.1 Thickness (in "two units) of the solid g7 targets used for the simulations ...................... 54

3.2 Number of hits in the drift chambers per sector and per region for $10^{5}$ bremstrahlung photons hitting the g7 target with energies ranging from $100 \mathrm{MeV}$ to $2.4 \mathrm{GeV}$. Case with $\mathrm{NO}$ mini torus in ................... 64

3.3 Number of hits in the drift chambers per sector and per region for $10^{5}$ bremstrahlung photons hitting the g7 target with energies ranging from $100 \mathrm{MeV}$ to $2.4 \mathrm{GeV}$. Left:case with $25 \%$ of the maximum mini torus current, Right: case with $-25 \%$. . . . . 65

3.4 Number of hits in the drift chambers per sector and per region for $10^{5}$ bremstrahlung photons hitting the $\mathrm{g} 7$ target with energies ranging from $100 \mathrm{MeV}$ to $2.4 \mathrm{GeV}$. Left:case with $50 \%$ of the maximum mini torus current, Right: case with $-50 \%$. . . 65

3.5 Number of hits in the drift chambers per sector and per region for $10^{5}$ bremstrahlung photons hitting the g7 target with energies ranging from $100 \mathrm{MeV}$ to $2.4 \mathrm{GeV}$. Left:case with $75 \%$ of the maximum mini torus current, Right: case with $-75 \%$. . . . 66

3.6 Number of hits in the drift chambers per sector and per region for $10^{5}$ bremstrahlung photons hitting the g7 target with energies ranging from $1 \mathrm{MeV}$ to $2.4 \mathrm{GeV}$. Case with $\mathrm{NO}$ mini torus in ........................ 66 
3.7 Number of hits in the drift chambers per sector and per region for $10^{5}$ bremstrahlung photons hitting the $\mathrm{g} 7$ target with energies ranging from $1 \mathrm{MeV}$ to $2.4 \mathrm{GeV}$. Left:case with $25 \%$ of the maximum mini torus current, Right: case with $-25 \% \ldots \ldots 67$

3.8 Number of hits in the drift chambers per sector and per region for $10^{5}$ bremstrahlung photons hitting the g7 target with energies ranging from $1 \mathrm{MeV}$ to $2.4 \mathrm{GeV}$. Left:case with $50 \%$ of the maximum mini torus current, Right: case with $-50 \% \ldots \ldots 67$

3.9 Number of hits in the drift chambers per sector and per region for $10^{5}$ bremstrahlung photons hitting the $\mathrm{g} 7$ target with energies ranging from $1 \mathrm{MeV}$ to $2.4 \mathrm{GeV}$. Left:case with $75 \%$ of the maximum mini torus current, Right: case with $-75 \% \ldots \ldots 8$

3.10 Number of hits in the drift chambers per sector and per region for $10^{5}$ bremstrahlung photons hitting the g7 target with energies ranging from $10 \mathrm{keV}$ to $2.4 \mathrm{GeV}$. Case with NO mini torus in ...................... 68

3.11 Number of hits in the drift chambers per sector and per region for $10^{5}$ bremstrahlung photons hitting the g7 target with energies ranging from $10 \mathrm{keV}$ to $2.4 \mathrm{GeV}$. Left:case with $25 \%$ of the maximum mini torus current, Right: case with $-25 \%$. . . . 69

3.12 Number of hits in the drift chambers per sector and per region for $10^{5}$ bremstrahlung photons hitting the g7 target with energies ranging from $10 \mathrm{keV}$ to $2.4 \mathrm{GeV}$. Left:case with $50 \%$ of the maximum mini torus current, Right: case with $-50 \%$. . . . 69

3.13 Number of hits in the drift chambers per sector and per region for $10^{5}$ bremstrahlung photons hitting the g7 target with energies ranging from $10 \mathrm{keV}$ to $2.4 \mathrm{GeV}$. Left:case with $75 \%$ of the maximum mini torus current, Right: case with $-75 \% \ldots \ldots 70$ 


\section{LIST OF FIGURES}

1.1 Inclusive $e^{+} e^{-}$mass spectra in $450 \mathrm{GeV}$ p-Be collisions at CERES . . 5

1.2 Inclusive $e^{+} e^{-}$mass spectra in $450 \mathrm{GeV}$ p-Au collisions at CERES . . 6

1.3 Inclusive $e^{+} e^{-}$mass spectra in $200 \mathrm{GeV} /$ nucleon $\mathrm{p}-\mathrm{Au}$ collisions at CERES .................... 6

1.4 Feynman diagrams of Vector Meson Photoproduction off nuclei . . . 12

2.1 Aerial view of the accelerator site . . . . . . . . . . 15

2.2 Schematic of CEBAF at $4 \mathrm{GeV}$ (design goal achieved in 1997) . . . 17

2.3 Schematic of the $12 \mathrm{GeV}$ upgrade of CEBAF . . . . . . . 18

2.4 The new seven cell cavity design for CEBAF at $12 \mathrm{GeV} \ldots \ldots$. . . 21

2.5 Side view of the CLAS detector in Hall B with beam line and associated equipment . . . . . . . . . . . . 23

2.6 Schematic of Hall B . . . . . . . . . . . . . . . . . . . . 24

2.7 Photon beam production and tagging . . . . . . . . 25

2.8 The CLAS detector, top view, cut along the beam line . . . . . . 30

2.9 The CLAS detector, cut perpendicular to the beam line . . . . . . . 31

2.10 Contours of constant absolute magnetic field for the CLAS toroid in the midplane between coils . . . . . . . . . . . 32

2.11 Magnetic field vectors for the CLAS toroid transverse to the beam in a plane centered on the target . . . . . . . . . . . . 33

2.12 Representation of portion of a region III sector of the drift chambers showing the layout of its two superlayers . . . . . . . 35

2.13 Schematic representation of a typical drift chamber sector . . . . . 37 
2.14 Optical arrangment of two of the modules of the CLAS Cerenkov detector ....................... 40

2.15 Schematic drawing of the array of optical modules in one of the six sectors of CLAS . . . . . . . . . . . . 40

2.16 Example of $\beta / p$ plot used to do particle identification . . . . . . . . 41

2.17 Schematic drawing of one sector of the time of flight system . . . . 42

2.18 Vertical section of an electromagnetic calorimeter module with its read-out systems . . . . . . . . . . . . . . 44

2.19 Exploded view of one of the six CLAS electromagnetic calorimeter modules . . . . . . . . . . . . . . . . 4 45

2.20 Event reconstruction in the electromagnetic calorimeter . . . . . . . 45

2.21 Geometry of one LAC module . . . . . . . . . . . . . . . 47

3.1 A drawing of the $\mathrm{g} 7$ target $\ldots \ldots \ldots \ldots \ldots \ldots$

3.2 Number of outgoing $e^{-}$or $\pi^{-}$versus the z-position of the vertex for events of the type $\gamma p \rightarrow \rho p \rightarrow e^{+} e^{-} p$ or $\gamma p \rightarrow$ $\rho p \rightarrow \pi^{+} \pi^{-} p$ with Gaussian fit . . . . . . . . . . 56

3.3 Number of outgoing $e^{-}$or $\pi^{-}$versus the z-position of the vertex for a g1c empty target run with gaussian fit . . . . . . . 57

3.4 Counts versus the invariant mass squared of the $e^{+} e^{-}$pairs for generated and reconstructed events of the type $\gamma p \rightarrow \rho p \rightarrow e^{+} e^{-} p \ldots 58$

3.5 Acceptance versus the invariant mass squared of the $e^{+} e^{-}$ pairs for events of the type $\gamma p \rightarrow \rho p \rightarrow e^{+} e^{-} p \ldots \ldots \ldots$

3.6 Counts versus the missing mass squared off of the $e^{+} e^{-}$pair (Mee2) with vertical lines illustrating the cut $M e e 2 \leq 0.85 \mathrm{GeV}^{2} / \mathrm{c}^{4}$ or $M e e 2 \geq 1 G e V^{2} / c^{4} \ldots \ldots \ldots \ldots \ldots \ldots \ldots$

3.7 Counts versus the z-position of the vertex for the "bad events" as defined in text or illustrated in Figure $3.6 \ldots \ldots$. . . . . 60

3.8 Photon total cross sections as a function of energy in carbon and lead, showing the contributions from different processes . . . . 62

3.9 Probability $\mathrm{P}$ that a photon interaction will result in an $e^{+} e^{-}$pair . . 63

3.10 The accumulated low energy $e^{+} e^{-}$pair background for 1000 incoming bremstrahlung photons with energies between $1 \mathrm{MeV}$ and $2.4 \mathrm{GeV}$. Case with $\mathrm{NO}$ mini torus in . . . . . . . . . 71 
3.11 The accumulated low energy $e^{+} e^{-}$pair background for 1000 incoming bremstrahlung photons with energies between $1 \mathrm{MeV}$ and $2.4 \mathrm{GeV}$. Case with $75 \%$ of the maximum mini torus current . . 71

3.12 The accumulated low energy $e^{+} e^{-}$pair background for 1000 incoming bremstrahlung photons with energies between $1 \mathrm{MeV}$ and $100 \mathrm{MeV}$. Case with NO mini torus in . . . . . . . . . 72

3.13 The accumulated low energy $e^{+} e^{-}$pair background for 1000 incoming bremstrahlung photons with energies between $1 \mathrm{MeV}$ and $100 \mathrm{MeV}$. Case with $75 \%$ of the maximum mini torus current . . 72

3.14 The accumulated low energy $e^{+} e^{-}$pair background for 1000 incoming bremstrahlung photons with energies between 100 $\mathrm{MeV}$ and $1 \mathrm{GeV}$. Case with $\mathrm{NO}$ mini torus in . . . . . . . . 73

3.15 The accumulated low energy $e^{+} e^{-}$pair background for 1000 incoming bremstrahlung photons with energies between 100 $\mathrm{MeV}$ and $1 \mathrm{GeV}$. Case with $75 \%$ of the maximum mini torus current 73

3.16 The accumulated low energy $e^{+} e^{-}$pair background for 1000 incoming bremstrahlung photons with energies between $1 \mathrm{GeV}$ and $2.4 \mathrm{GeV}$. Case with $\mathrm{NO}$ mini torus in . . . . . . . . . 74

3.17 The accumulated low energy $e^{+} e^{-}$pair background for 1000 incoming bremstrahlung photons with energies between $1 \mathrm{GeV}$ and $2.4 \mathrm{GeV}$. Case with $75 \%$ of the maximum mini torus current . . 74

3.18 Number of $e^{-}$or $\pi^{-}$particles versus the scattering angle $\theta \ldots 75$

3.19 Number of $e^{+}$or $\pi^{+}$particles versus the scattering angle $\theta \ldots \ldots 76$

3.20 Counts versus the opening angle between the outgoing $e^{+} e^{-}$pairs $\ldots 76$

3.21 Counts versus the $e^{+} e^{-}$invariant mass squared for events of the type $\gamma p \rightarrow \rho p \rightarrow e^{+} e^{-} p \ldots \ldots \ldots \ldots \ldots \ldots$

3.22 Counts versus the invariant mass squared of those $e^{+} e^{-}$pairs that give hits in the same sector of CLAS, for events of the type $\gamma p \rightarrow \rho p \rightarrow e^{+} e^{-} p \ldots \ldots \ldots \ldots \ldots \ldots$ 


\section{Chapter 1}

\section{Introduction}

The work for this thesis essentially consisted of doing simulations for the E01112 experiment, or more familiarly called the g7 experiment, prior to its run at the TJNAF (Thomas Jefferson National Accelerator Facility), also known as the Jefferson Lab or simply Jlab. The terms 'g7' and 'Jlab' will be used in the rest of the text.

g7, an $A^{-}$rated experiment at Jlab, took place in Hall-B September 20, 2002 through November 13, 2002. The analysis of its data shall be the subject of my $\mathrm{PhD}$ thesis. The experiment made use of the accelerator facility CEBAF (Continuous Electron Beam Accelerator Facility) of Jlab and the CLAS detector (CEBAF Large Acceptance Spectrometer) located in Hall-B. Both of them will be described in detail in the next chapter.

A test run for $\mathrm{g} 7$ took place June 15, 2002 and will be the subject of the last chapter of the present thesis due to its relevance to the background simulations. 


\subsection{What is g7 about?}

The g7 experiment was proposed for the first time at Jlab in 1994 by P.Y. Bertin, M. Kossov and B.M. Preedom ([Ber94]) based on the ideas contained in the 2 letters of intent LOI-89-001 by P.Y. Bertin and P.A.M. Guichon, and LOI-93-004 by B.M. Preedom and P.Y. Bertin. It was accepted by the PAC9 (Program Advisory Commitee number 9), with a $B^{+}$rating, but never ran due to a lack of manpower. It was resubmitted, to PAC20, in 2001 by C. Djalali, D.P. Weygand, and M. Kossov ([Dja01]), and was accepted again, with an $A^{-}$ rating. It successfully ran in the fall of 2002. The experiment has been designed to measure the properties of vector mesons inside nuclear matter. Indeed, the properties of vector mesons, like their mass and width, are predicted to change at high baryon density, as will be described in more detail in the following section.

The goal of the experiment is to examine the inclusive $e^{+} e^{-}$photoproduction in the incoherent region. The reaction of interest to $\mathrm{g} 7$ is

$$
\gamma A \rightarrow V A^{\prime} \rightarrow e^{+} e^{-} A^{\prime}
$$

where $\mathrm{V}$ could be a $\rho$, an $\omega$ or a $\phi$ meson.

The electrons delivered by the accelerator emit bremstrahlung photons by passing through a radiator. The photon beam for g7 was made of these tagged bremstrahlung photons. The experiment ran at a $3 \mathrm{GeV}$ electron beam energy for most of its run period (four fifth of the total run time) and at a $4 \mathrm{GeV}$ electron beam energy at the end. Bremstrahlung photons generated by a $3 \mathrm{GeV}$ electron beam for instance, should have energies ranging from practically zero to $3 \mathrm{GeV}$. g7 should therefore have been able to produce $\rho, \omega$ and $\phi$ mesons. But the $\rho$ meson is the most interesting of the three, since it is the vector meson for which the most significant property modification is expected, if it indeed occurs. The choice of the energy ranges was motivated by the desire to be as close to the $\rho$ production threshold as possible to give it as little chance as possible of escaping from the nucleus before it decayed. The photon beam energy threshold 
to produce a $\rho$ on a proton is $1.086 \mathrm{GeV}$ (this threshold is $1.108 \mathrm{GeV}$ for an $\omega$ and $1.574 \mathrm{GeV}$ for a $\phi)$.

One of the major challenges in the analysis of this experiment will be to separate the $e^{+} e^{-}$events from the very large hadronic "background" (i.e. decay into two pions). Cuts on the energy deposited in the electromagnetic calorimeter, the Cerenkov counter signal and the transverse momentum, should give sufficient $e^{+} e^{-} / \pi^{+} \pi^{-}$discrimination as shown in the CLAS-NOTE-2001-009 ([Kos01]). Now, one should also be able to separate the incoherent production from the coherent background. The $d \sigma / d \Omega$ for the coherent production is very forward peaked, resulting in a very small momentum transfer to the struck nucleus. Detecting the recoiling nucleon can help in suppressing the coherent production while a kinematical cut in -t should substancially enhance the incoherent production (coherent production happens typically at very low $-\mathrm{t}$ ).

\subsection{Change of the properties of the vector mesons inside nuclear matter: a historical overview}

A few conjectures of the late eighties, more rigorously justified in the early nineties came out predicting a decrease in the mass of the vector mesons at high baryon density, possibly due to a partial restoration of chiral symmetry, and an increase in their width due to collisional broadening. By using effective chiral Lagrangians with a suitable incorporation of the scaling property of QCD, G. E. Brown and M. Rho ([Bro91]) proposed the in medium scaling law

$$
m_{\rho}^{*} / m_{\rho} \approx m_{\omega}^{*} / m_{\omega} \approx f_{\pi}^{*} / f_{\pi} \approx 0.8
$$

where $f_{\pi}$ is the $\pi \rightarrow \mu \nu$ decay constant playing the role of an order parameter for the chiral symmetry restoration and where the starred quantities denote the in-medium mass of the $\rho$ and $\omega$ mesons or the in-medium pion decay constant, whereas the unstarred ones refer to the $\rho$ and $\omega$ masses in vacuum or 
the in-vacuum pion decay constant. This law had been conjectured earlier by G. E. Brown ([Bro88]) and predicts a $20 \%$ drop in the vector meson mass at normal nuclear density. Not much after the paper by G. E. Brown and M. Rho, T. Hatsuda and S. H. Lee ([Hat91]) found a linear decrease of the vector meson masses as a function of the density, using calculations based on QCD sum rules at low density. According to their model, the four-quark condensate $\left\langle(q \bar{q})^{2}>\right.$ and the two-twist condensate, $<\bar{q} \gamma_{\mu} \bar{q}_{\mu} D_{\nu} q>$ play dominant roles for the mass-shift of light mesons. This shift is expressed in the formula

$$
m^{*} / m \approx 1-C\left(\rho / \rho_{0}\right)
$$

where $\mathrm{C} \approx 0.18$ for the $\rho$ and $\omega$-mesons and where $\rho_{0}$ denotes normal nuclear density (about $0.17 \mathrm{fm}^{-3}$ ) and $\rho$, an increased nuclear density, as that might be the case in heavy ion collisions. Thus, they predict an $18 \%$ drop in the vector meson masses at normal nuclear density. In 1990, P. Y. Bertin and P. A. M. Guichon published an idea for an experiment to 'detect the quark effects in nuclei' ([Ber90]), that is to actually verify the dropping of the mass of the $\rho$ in the nucleus, by sending on a lead target, a bremstrahlung photon beam generated by $2 \mathrm{GeV}$ electrons and looking at the $e^{+} e^{-}$decay channel. They devised the experiment after P. A. M. Guichon pointed out a $120 \mathrm{MeV}$ downward shift of the meson mass using a very simplistic model in 1989 ([Gui89]). The same kind of conclusion concerning the $\rho$ meson mass in medium was reached by M. Asakawa and C. M. Ko ([Asa93]) a little later in 1993, again using calculations based on QCD sum rules. By about the same time, C. M. Shakin and Wei-Dong Sun, were finding about a $20 \%$ decrease in the mass of the $\rho$ and a $35 \%$ reduction of its width at nuclear matter density using the Nambu-Jona-Lasino model ([Sha93]). Their conclusion about the width of the $\rho$ deviates from the previous predictions ([Ber90] for instance) which pointed out an increase. Many of the ideas contained in the reference [Ber90] have been used later to devise g7.

An important experimental piece of information came in 1995 from the CERES 


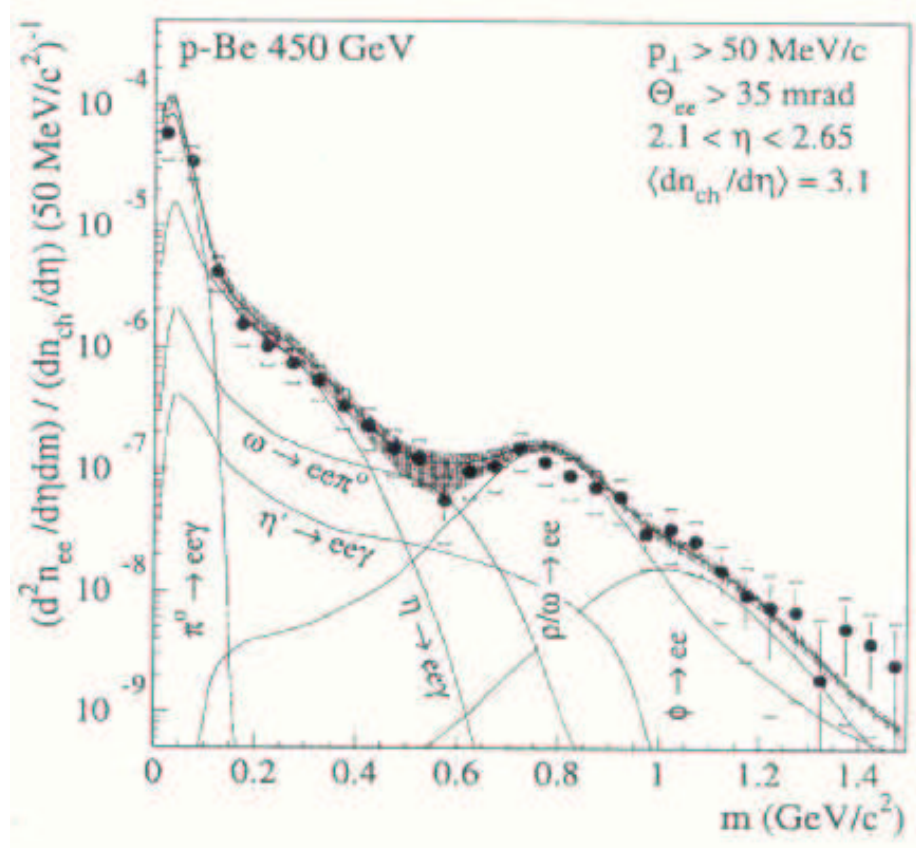

Figure 1.1: Inclusive $e^{+} e^{-}$mass spectra in $450 \mathrm{GeV}$ p-Be collisions at CERES showing the data and the various contributions from hadron decays ([Aga95]). The shaded region indicates the systematic error on the summed contributions.

([cer]) and HELIOS/3 Collaborations of CERN. Early that year, the CERES Collaboration reported on measurements of low-mass electron pairs in $450 \mathrm{GeV}$ p-Be, p-Au, and $200 \mathrm{GeV} /$ nucleon S-Au collisons at central rapidities ([Aga95]). While, for the proton induced data, within the systematic errors, they were able to satisfactorily explain the low-mass spectra by electron pairs from hadron decays, an enhancement over the hadronic contributions by roughly a factor of 5 in the invariant mass range $0.2<m<1.5 \mathrm{GeV} / c^{2}$ was observed for the $\mathrm{S}-\mathrm{Au}$ system. The onset of the excess was found to be around $2 m_{\pi}$, suggesting that it arose from two-pion annihilation $\pi \pi \rightarrow e^{+} e^{-}$. The Figures 1.1, 1.2 and 1.3 illustrate these results.

The HELIOS/3 Collaboration also reported an excess production of dileptons in the low-mass region ([Mas95]). The latter measured dimuon production in $200 \mathrm{GeV} /$ nucleon S-W collisions and $200 \mathrm{GeV}$ p-W collisions at more forward rapidities. The onset of the excess was similarly found to be around $m_{\mu \mu} \approx 2 m_{\pi}$. 


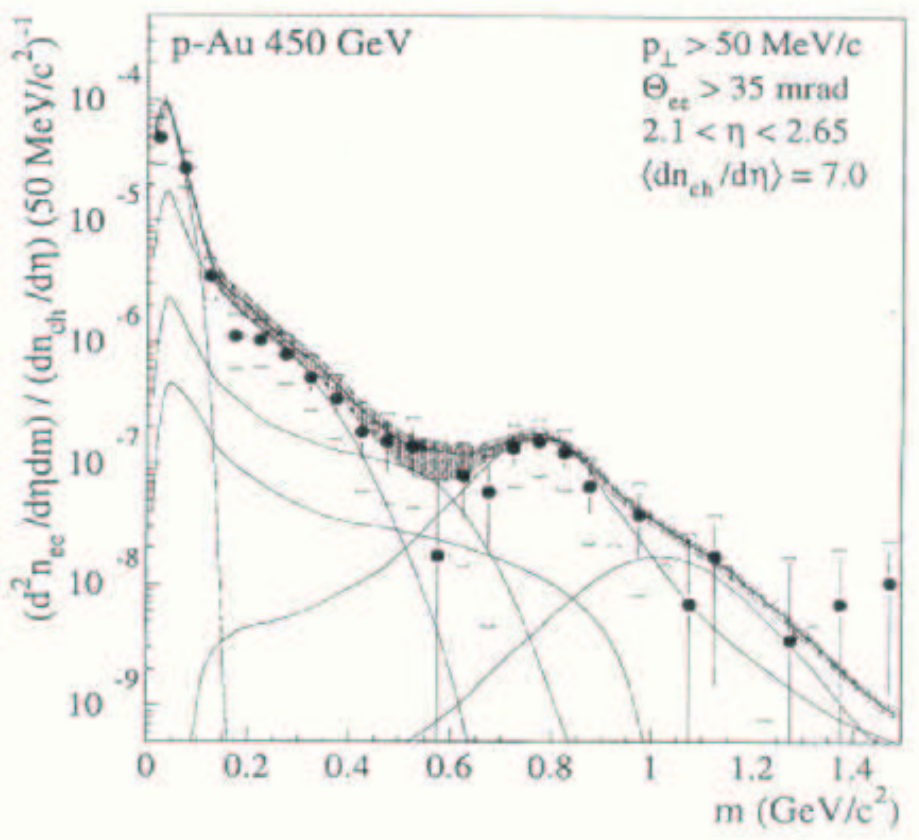

Figure 1.2: Inclusive $e^{+} e^{-}$mass spectra in $450 \mathrm{GeV}$ p-Au collisions at CERES. Same explanations as for 1.1.

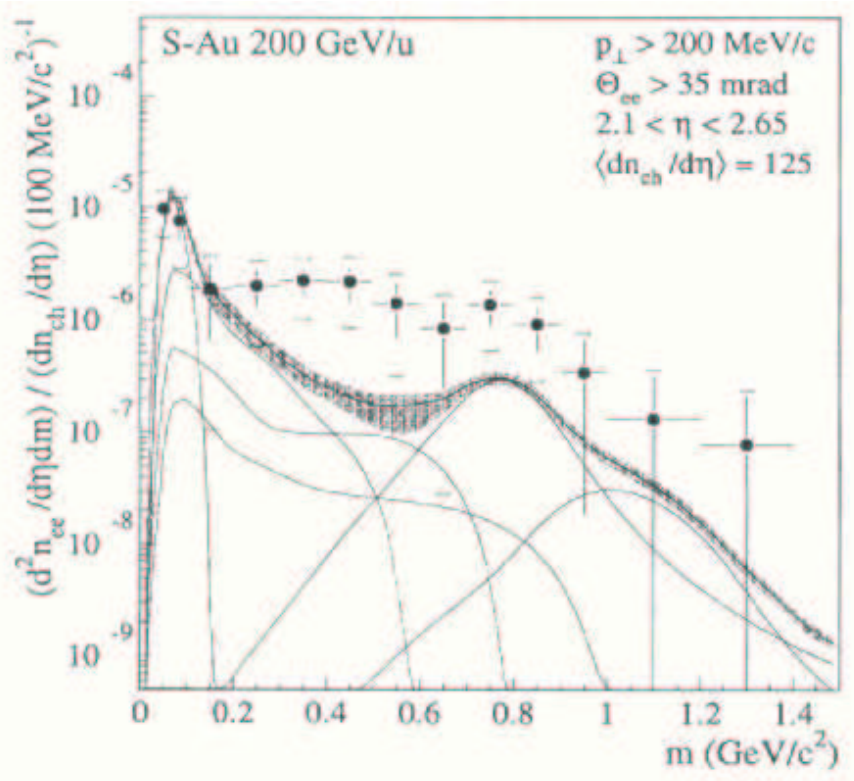

Figure 1.3: Inclusive $e^{+} e^{-}$mass spectra in $200 \mathrm{GeV} /$ nucleon $\mathrm{S}+\mathrm{Au}$ collisions at CERES. Same explanations as for 1.1. 
However, the magnitude of the enhancement factor (the integral of the S-W data over the integral of the $\mathrm{p}-\mathrm{W}$ data in the comparable mass range) was found to be significantly smaller (about 1.6). The difference was attributed to a non-linear dependence of the underlying production mechanism.

A few months later in 1995, a paper by G. Q. Li, C. M. Ko and G. E. Brown was published ([Li95]), in which the authors claimed that, by using a relativistic transport model for the expansion stage of $\mathrm{S}+\mathrm{Au}$ collisions at $200 \mathrm{GeV} /$ nucleon, they were able to account for the enhancement of low-mass dileptons observed by the CERES Collaboration, if they introduced a decrease of the vector meson masses in hot and dense matter. The same idea came forth in yet another publication that year ([Cas95]). However, it has been argued that more conventional effects to explain the CERES and HELIOS data can not be ruled out. Thus more conservative effects, such as a modified in-medium pion dispersion relation may give sufficient enhancement ([Son96, Cha96]). Another paper published in 1996, by V. Koch and C. Song ([Koc96]), also supports this idea. The authors of the latter reference claimed that, with some initial conditions (not including medium modification), they were able to come within the lower end of the sum of statistical and systematic error of the CERES data and at the same time obtain a reasonable agreement with the HELIOS data. They furthermore found that if they considered some in-medium modification, it only gave an insignificant enhancement of the total dilepton yield. Other papers that appeared later ([Cas98]), were revealing little difference between the 'hadronic' and the 'dropping mass' scenarios in accounting for the CERES and HELIOS/3 data, confirming the idea that a clear discrimination between the two scenarios was impossible at the time.

Early 1997, the result of a study of the energy dependence of the mass shift of vector mesons was published by V. L. Eletsky and B. L. Ioffe ([Ele97]). They found that for $2 \mathrm{GeV} \leq E_{\rho} \leq 7 \mathrm{GeV}$, the mass shift of the $\rho$ is positive, i.e. the $\rho$ mass increases. The authors furthermore stated that a direct comparison of 
their results with the previous calculations was impossible, given that the latter essentially focused on the mass shift of the $\rho$ for the energy range $E_{\rho} \leq 1 \mathrm{GeV}$. Later in 1998, another paper was published ([Kon98]), where the authors found a dropping of the $\rho$ mass for $p \approx 0$ ( $\rho$ produced at rest) roughly in line with the QCD sum rule calculation by Hatsuda and Lee mentioned ealier, and an increase of the $\rho$ mass at high energy close to the prediction by Eletsky and Ioffe, thus demonstrating the compatibility of the two results.

A paper was published in 1999 by M. Effenberger, E. L. Bratkovskaya and U. Mosel ([Eff99]), where the authors presented the $e^{+} e^{-}$invariant mass spectra for $\gamma \mathrm{C}, \gamma \mathrm{Ca}$, and $\gamma \mathrm{Pb}$ at $0.8,1.5$ and $2.2 \mathrm{GeV}$, based on a calculation using a semiclassical Boltzmann-Uehling-Uhlenbeck (BUU) transport model, which should be of interest for the analysis of the g7 data since they focused on observable effects of medium modification of the $\rho$ and the $\omega$ mesons.

Two more experiments reported results recently, relevant to the search on medium modification of vector meson properties. The first results were published by a group from the TAGX collaboration at the Institute for Nuclear Study (INS) of the University of Tokyo ([Lol98], [Kag99], [lol]). They claimed having observed a mass shift as large as $160 \pm 35 \mathrm{MeV} / c^{2}$ in the mass of the $\rho$ via the ${ }^{3} \operatorname{He}\left(\gamma, \pi^{+} \pi^{-}\right) \mathrm{X}$ reaction. But, there are two problems with the reported results. The first one is that, although the $\pi^{+} \pi^{-}$channel is the preferred decay channel of the $\rho$ (the branching ratio is almost 100\%), the very large number of final state interactions of the hadronic pair make it difficult to clearly interpret the results. The second is that, the mass shift reported is suprisingly huge for $\rho$ mesons produced in such a small nucleus as the ${ }^{3} \mathrm{He}$ and do not match any theoretical predictions for this nucleus published so far. The second set of results were published on the KEK-PS E325 experiment ([Oza01], [En'00], [Oza02]). Invariant mass spectra of the $e^{+} e^{-}$pairs in the target rapidity region of $12-\mathrm{GeV}$ $\mathrm{p}+\mathrm{A}$ reactions were measured. The experiment suffered from a lack of statistics though and the only conclusions that the authors were able to come up with was 
that the spectral shape of the $\rho, \omega$ and $\phi$ mesons is modified at normal-matter density.

The change of the properties of the vector mesons in dense medium is a hot topic that will be further studied at RHIC(BNL) ([rhi]), ALICE(CERN) ([ali]) and HADES(GSI) ([had]) by measuring the low mass dilepton production, in addition to g7 at TJNAF. The HADES detector is a dedicated high acceptance spectrometer to measure dielectrons with invariant masses up to $1 \mathrm{GeV} / c^{2}$ in $\pi+A, p+A$ and $A+A$ reactions. More results could come from the TAGX collaboration on the $\pi^{+} \pi^{-}$channel for bigger nuclei. The spring- 8 collaboration has an experiment to study the $\gamma+\mathrm{A} \rightarrow \phi+\mathrm{X}\left(\phi \rightarrow K^{+} K^{-}\right)$. And the KEK-PS group could come up with more statistics for the reactions on which they already published results. All of these experiments should yield results complementary to each other, that will hopefully help understand what happens to the vector mesons properties in dense medium.

\subsection{Why a photon induced reaction?}

The reference [Eff99] gives a few reasons why photon or pion induced reactions are particularly interesting. First of all, since in heavy-ion collisions the final dilepton yield is obtained by an integration over dileptons emitted at different densities and temperatures, a discrimination between the different scenarios of in-medium modification to explain the low-mass dilepton yield is difficult. Moreover, the ultra-relativistic heavy ion collisions proceed, at least in their initial stages, far from equilibrium, whereas all theoretical predictions of in-medium properties are based on equilibrium assumptions. The photon or pion induced reactions offer "cleaner" conditions to study the properties of vector mesons in medium, since in such reactions the nuclear medium is very close to equilibrium at normal nuclear density and zero temperature. Furthermore, in photon-nucleus reactions, the produced particles have in general larger momenta with respect 
Table 1.1: Characteristics of the $\rho, \omega$ and $\phi$ mesons

\begin{tabular}{llll}
\hline \hline Parameter & $\rho$-meson & $\omega$-meson & $\phi$-meson \\
\hline \hline Lepton branching ratio & $e^{+} e^{-}(0.0044 \%)$ & $e^{+} e^{-}(0.0072 \%)$ & $e^{+} e^{-}(0.0031 \%)$ \\
Hadron branching ratio & $2 \pi(100 \%)$ & $3 \pi(89 \%), 2 \pi(2 \%)$ & $2 \mathrm{~K}(84 \%), 3 \pi(12 \%)$ \\
$m_{V}(\mathrm{MeV})$ & 771.1 & 782.57 & 1019.45 \\
$\Gamma_{V}(\mathrm{MeV})$ & 149.2 & 8.44 & 4.26 \\
$\mathrm{c} \tau(\mathrm{fm})$ & 1.3 & 23.4 & 44.4 \\
$\sigma_{V N}(\mathrm{mb})$ & 21 & 21 & 12 \\
\hline \hline
\end{tabular}

to the nuclear environment than in heavy-ion collisions ([Eff00]). Therefore, photonuclear experiments yield partly complementary information about the inmedium self-energies of mesons compared to heavy-ion experiments.

\subsection{Why the rho meson production?}

This section is largely inspired from the 1994 proposal for g7 ([Ber94]). The characteristic features of vector mesons are given in Table 1.1. This table contains not only the well known lepton and hadron branching ratios, but also the vector meson masses and widths, the decay length $(\mathrm{c} \tau)$, and the cross section for vector meson interaction with nuclear matter.

For g7, one would expect the maximum count rate at $E_{\gamma}=1.5 \mathrm{GeV}$ where both $\rho$ and $\omega$ production cross sections reach their maximum values. For this energy, all length scales are boosted by a factor of two. Thus, the decay length is expected to be $2.5 \mathrm{fm}$ for the $\rho$-meson, $45 \mathrm{fm}$ for the $\omega$-meson and $85 \mathrm{fm}$ for the $\phi$-meson. These lengths should be compared to the radii of the target nuclei: 2.5 $\mathrm{fm}$ for the carbon, $4.5 \mathrm{fm}$ for the iron and $6.5 \mathrm{fm}$ for the lead. A simple calculation shows that $63 \%$ of the $\rho$-mesons will decay inside the carbon, $83 \%$ inside the iron and $93 \%$ inside the lead. For the narrow vector mesons, the situation is different: 
even inside the lead, only $13 \%$ of the $\omega$-mesons and $7 \%$ of the $\phi$-mesons will decay in medium. Actually, according to Bertin and Guichon ([Ber90]), the $\phi$-meson is not expected to be shifted, because in first approximation, the $\phi$ does not couple to the nuclear field. It should thus be a good reference point for the analysis of g7. All in all, because the $\rho$-meson has such a short lifetime compared to the $\omega$ or $\phi$-mesons and will therefore essentially decay inside the nucleus once produced there, it is the ideal vector meson to probe for changes in the properties of vector mesons in medium.

\subsection{Why the leptonic decay channel?}

In the year 2000, a paper published by M. Effenberger and U. Mosel ([Eff00]), gave predictions for the $\pi^{+} \pi^{-}$invariant mass spectrum in $\gamma \mathrm{C}$ and $\gamma \mathrm{Pb}$ reactions, using a BUU transport model including a dropping of the meson masses. The outcome was that the $\pi^{+} \pi^{-}$spectrum is hardly influenced by such a medium modification. This is simply due to the fact that the pions have a very short mean free path in the nuclear medium (about $1 \mathrm{fm}$ ). Therefore the probability that two pions that stem from a decay of a $\rho$-meson at normal nuclear density are both able to propagate to the vacuum without rescattering is very low. Thus, since the $e^{+} e^{-}$only interact electromagnetically and not strongly like the pions, they give a "clean" way to probe the properties of the vector mesons inside the nucleus.

\subsection{The production mechanism}

The Feynman diagrams for the photoproduction of the vector mesons are shown in Figure 1.4. The diffractive mechanism and the One Pion Exchange (OPE) mechanism give the bulk of the vector meson production.

It should be noted that the incoherent production (where the photon inter- 

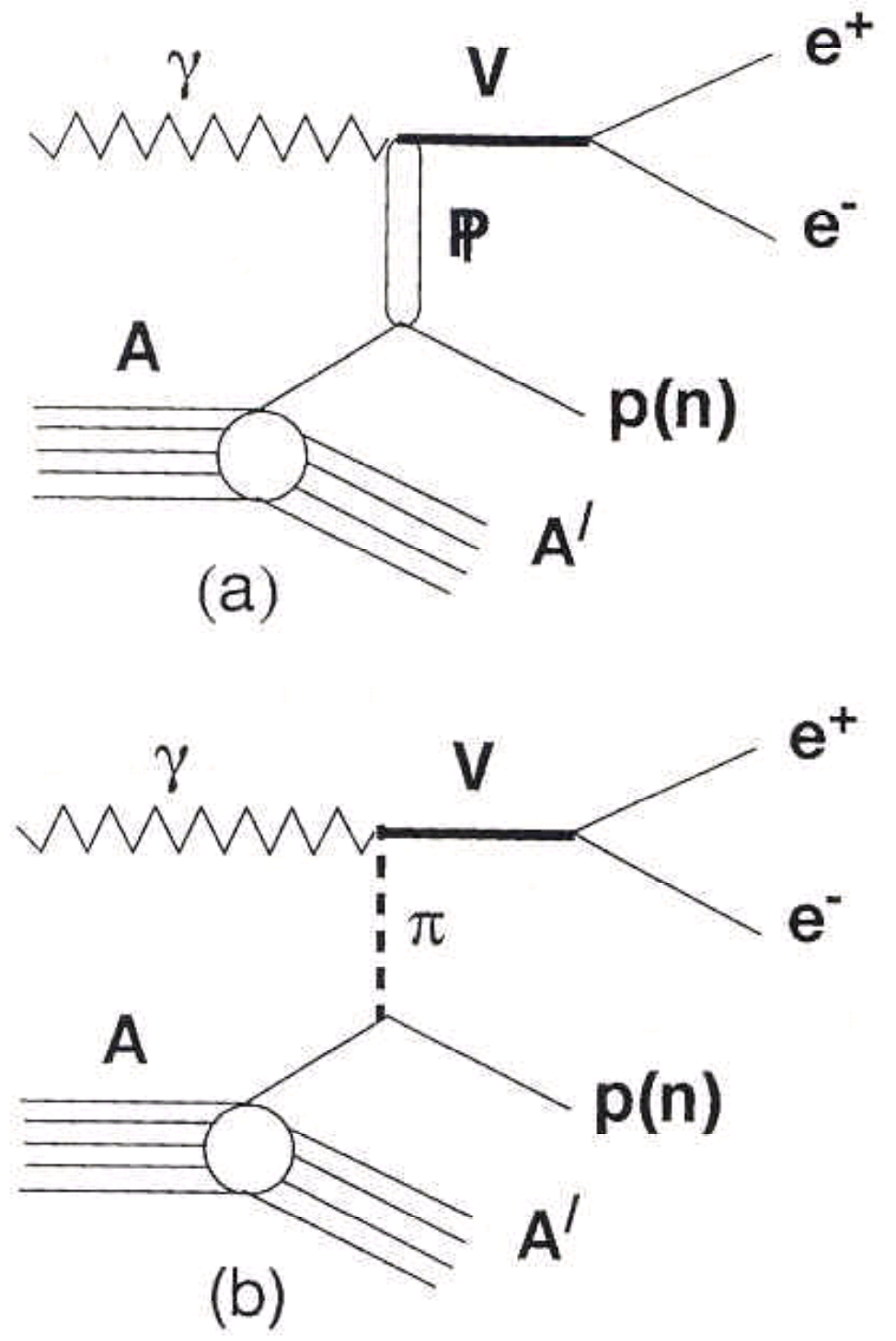

Figure 1.4: Feynman diagrams of Vector Meson Photoproduction off nuclei ([Ber94]): (a). Diffractive photoproduction. (b). One Pion Exchange mechanism of photoproduction. 
acts with the individual nucleons) is what matters to g7 and that the coherent production (where the photon "sees" the nucleus as a whole) only forms a background to be eliminated. Detecting a recoiling neutron or proton for a given event would be a clear signature of an incoherent process. In the case of the deuterium, the detection of a recoiling deuteron would indicate a coherent reaction. In the case where no recoiling particle is detected, a cut on -t could help discriminate between the two productions mechanisms (coherent processes happen typically at very low -t). Just as a remark, while the early seventies saw a remarkable number of experiments of photoinduced particle production (a rather complete list of which can be found in [Bau78]), the emphasis was on the study of the coherent production essentially. The incoherent production still has very few experimental data to rely on, as noted in [Eff00]. Hence again the need for experiments of the type of $\mathrm{g} 7$. 


\section{Chapter 2}

\section{The experimental setup}

Jlab is a nuclear physics research laboratory run by the Southeastern Universities Research Association for the Department of Energy in Newport News, Virginia. Its primary goal is to do basic research in order to better understand the atom's nucleus, but it conducts, as a secondary goal, some applied research as well, for using the Free-Electron Lasers (FEL) based on technology that Jlab developed for its own experiments.

For the purpose of basic research, Jlab houses a continuous electron beam accelerator, CEBAF, and 3 experimental halls, the Halls A, B and C. CEBAF was originally designed to deliver electrons with energies up to a maximum of 4 $\mathrm{GeV}$ (which was achieved in 1997), but, since 2000, it routinely delivers beam energies up to $6 \mathrm{GeV}$, which allows experiments at the nuclear and particle physics interface. There is a project of upgrading CEBAF to deliver beam energies up to $12 \mathrm{GeV}$ and to build an extra experimental hall, the Hall D. The Lab also has a nuclear theory group pursuing a broad program of theoretical research in sup-

port of its experimental physics program. A few of the topics of interest to the theory group are the study of the spectra and decays of hadrons and confinement 


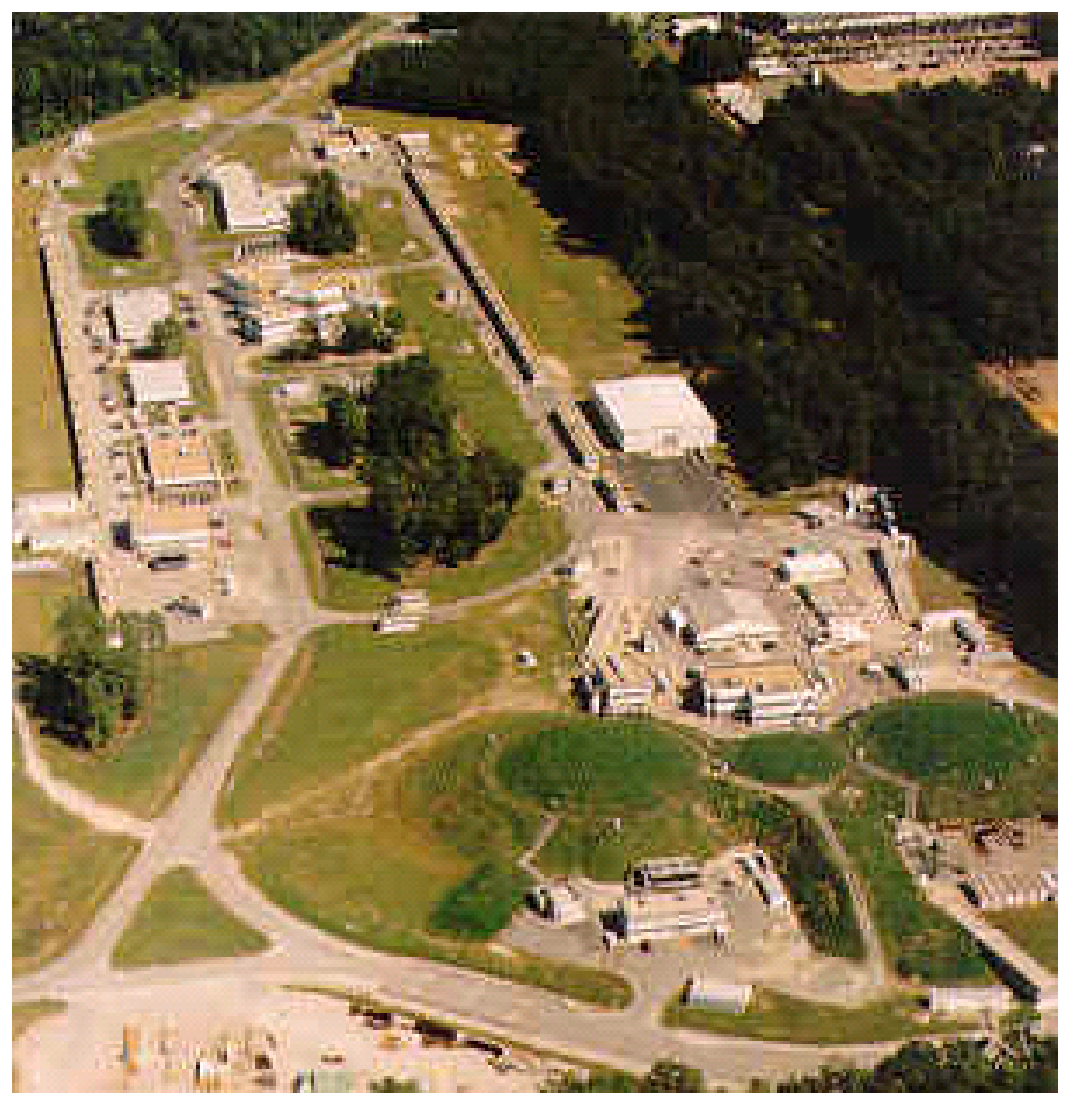

Figure 2.1: Aerial view of the accelerator site 
in QCD, the hadron form factors and quark-gluon distributions, the strangeness and parity violation in hadrons and nuclei, the few-body and many-body nuclear physics.

The three experimental halls have complementary facilities, and each has a different mode of operation ([Gil02]). Hall A is devoted to presicion measurements, and houses twin high-resolution spectrometers that can each be placed at different angles with respect to the beam. Hall $\mathrm{C}$ is similar, but is used for what Jlab calls major set-up experiments, those that require complex dedicated apparatus. Hall B contains the CLAS, a detector with a toroidal magnetic field configuration that covers $90 \%$ of $4 \pi$, and is run by a collaboration of approximately 200 scientists.

The following sections will give a description of CEBAF and Hall B, as these were the facilities/equipment that g7 used, and will contain references for further information.

\subsection{CEBAF: The continuous electron beam ac- celerator facility}

As CEBAF is continually beeing worked on and is subject to further modifications (cf. the $12 \mathrm{GeV}$ upgrade), the information given in this section, with the exception of the historical reminders, is true starting from early 2001 and holds up to the time this thesis was written and defended. A major source of inspiration for this section was the reference [Lee01], in which the interested reader can find a rather complete description of CEBAF from a historical and technical point of view. It also gives a nice list of the past physics achievements it enabled and the future research perspectives.

The original idea behind the proposal of CEBAF was to have an accelerator to study the interface between the nuclear and the particle physics, the transition region between the energy regime where strongly interacting matter is under- 


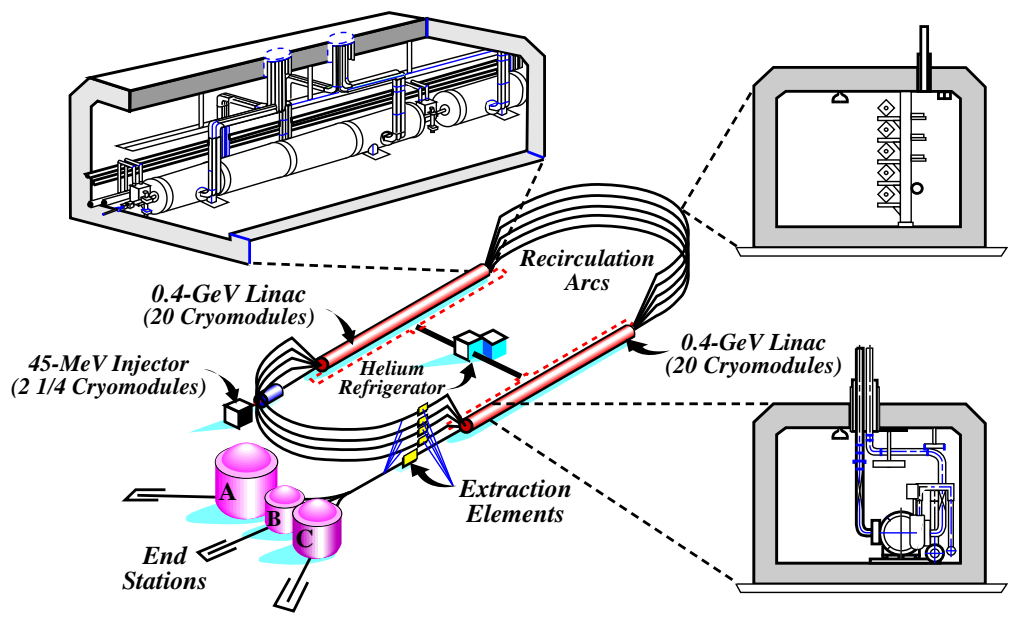

Figure 2.2: Schematic of CEBAF at $4 \mathrm{GeV}$ (design goal achieved in 1997). CEBAF can deliver up to $6 \mathrm{GeV}$ since the year 2000 .

stood as nucleon bound states and the regime where the underlying quark-gluon structure appears. This required a combination of characteristics: multi-GeV energy for spacial resolution and kinematic flexibility, high intensity for precise measurement of relatively small electromagnetic cross sections, high duty factor to allow coincidence experiments, and beam quality sufficient for use with highresolution spectrometers and detectors. Essential for the new accelerator were also: the continuous wave operation, currents up to $200 \mu \mathrm{A}$, and simultaneous delivery of beams to multiple users. The upper limit for the beam energy, as originally specified, was $4 \mathrm{GeV}$.

Those specifications where indeed met and gave birth to CEBAF, a five-pass recirculating superconducting radiofrequency LINAC, capable of simultaneous delivery of beam to three end stations of continuous wave beams of up to $200 \mu \mathrm{A}$ with $75 \%$ polarization (if needed) and a relative momentum spread of a few $10^{-5}$. CEBAF routinely delivers beam currents sufficient to achieve luminosities of several times $10^{38} \mathrm{~cm}^{-2} \mathrm{~s}^{-1}$ to Halls A and C. The properties of the Hall B detector, CLAS, however limit the maximum luminosity for this Hall, for which the beam current is four orders of magnitude smaller than that simultaneously delivered to the other Halls. The lowest operating energy is $0.6 \mathrm{GeV}$, the present full energy 


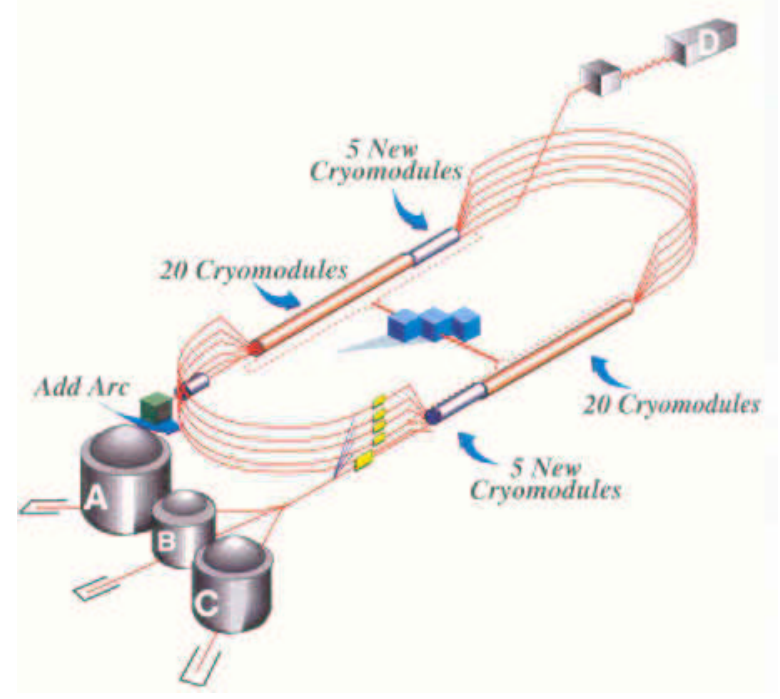

Figure 2.3: Schematic of the $12 \mathrm{GeV}$ upgrade of CEBAF.

is nearly $6 \mathrm{GeV}$, and an upgrade to $12 \mathrm{GeV}$ is possible and indeed planned. A schematic of CEBAF as it stands now is shown in Figure 2.2, while the Figure 2.3 shows a schematic of the proposed $12 \mathrm{GeV}$ upgrade. The combination of five-pass recirculation, a three-laser photocathode source, and subharmonicrf-separator-based extraction enables simultaneous delivery of three beams at different energies, with hall-to-hall current ratios approaching $10^{6}$, and with a specified orientation of the beam polarization. The total length of the accelerator is $1.4 \mathrm{~km}$.

The most important innovations in CEBAF are the choice of the superconducting radiofrequency technology and the use of multipass beam recirculation. Neither had been previously applied on the scale of CEBAF. Actually, until LEP II came into operation, CEBAF was the world's largest implementation of superconducting radiofrequency technology. Beam recirculation minimizes the cost of that implementation.

Simultaneous delivery of highly polarized and nominally unpolarized beam has become routine. The polarized source liberates electrons from a Cs and NF3 coated Galium Arsinide crystal by means of photo-emission. The duty-factor 
conserving three beam operation is implemented by creating three interlaced 499 $\mathrm{MHz}$ beams at the source (at $100 \mathrm{keV}$ ). Spaced apart by $120^{\circ}$ of rf phase, they form a $1497 \mathrm{MHz}$ beam in which each bunch has properties, that may differ from its immediately preceding and trailing pair of neighbors, but which repeats every third bunch. This is achieved by using three independent rf-gain-switched lasers directed at a single photocathode (the Galium Arsinide crystal). The beam formed this way has a 2.004 ns bunch structure and progresses into the injector (each laser "fires" every 2ns (=1 / $499 \mathrm{MHz}$ ) producing the beam for a given experimental hall). There is a spare gun in case the photocathode gets old or damaged and switching from the one to the other takes about 1 hour. The injector has 18 accelerating cavities, which originally were designed to boost the electrons up to $45 \mathrm{MeV}$ in energy, but have by the year 2000 been able to get them up to $67 \mathrm{MeV}$. The beam enters then into the north LINAC, made of 160 accelerating cavities, is then guided to the south LINAC by the east arc, where dipole magnets bend its tragectory. The LINACs were originally designed to give an extra $0.4 \mathrm{GeV}$ of energy to the electrons, but are by now able to go up to 0.6 $\mathrm{GeV}$. The beam particles get further accelerated in the south LINAC, exactly similar in design to the north LINAC, and enter the west arc to be led to the north LINAC again, and so forth, up to five passes total in each LINAC. In the arcs, the electrons in the pipes closest to the floor have been around more times than those in pipes closest to the ceiling, and thus have a bigger energy and need stronger bending magnets. The electrons can be extracted by the end of any complete tour, with an energy equal to a multiple (between 1 and 5) of one fifth of the end point energy. Simultaneous distribution to the three end stations is achieved by using properly phased rf deflecting cavities ("rf separators") operating at $499 \mathrm{MHz}$ following the final pass through the accelerator. The main machine parameters are summarized in Table 2.1.

The accelerating cavities are made of pure niobium sheet and plate and have been carefully inspected for surface defects. Cavities were hermetically paired 
Table 2.1: Main machine parameters (from reference [Lee01]).

\begin{tabular}{ll}
\hline \hline Energy & $6.06 \mathrm{GeV}$ \\
Average current (Halls A and C) & $1-150 \mu \mathrm{A}$ \\
Average current (Hall B) & $1-100 \mathrm{nA}$ \\
Bunch charge & $<0.3 \mathrm{pC}$ \\
Repetition rate & $499 \mathrm{MHz} / \mathrm{hall}$ \\
Beam polarization & $>75 \%$ \\
Beam size (rms transverse) & $\approx 80 \mu \mathrm{m}$ \\
Bunch length (rms) & $300 \mathrm{fs}, 90 \mu \mathrm{m}$ \\
Energy spread & $2.5 \mathrm{x} 10^{-5}$ \\
Beam power & $<1 \mathrm{MW}$ \\
Beam loss & $<1 \mu \mathrm{A}$ \\
Number of passes & 5 \\
Number of accelerating cavities & 338 \\
Fundamental mode frequency & $1497 \mathrm{MHz}$ \\
Accelerating cavity effective length & $0.5 \mathrm{~m}$ \\
Cells/cavity & 5 \\
Average $Q_{0}$ & $4.0 \times 10^{9}$ \\
Implemented $Q_{\text {ext }}$ & $5.6 \times 10^{6}$ \\
Cavity impedance (r/Q) & $980 \Omega$ \\
Average cavity accelerating gradient & $7.5 \mathrm{MV} / \mathrm{m}$ \\
RF power & $<3.5 \mathrm{~kW} / \mathrm{cavity}$ \\
Amplitude control & $1.00 \mathrm{x} 10^{-4} \mathrm{rms}$ \\
Phase control & $0.1^{\circ} \mathrm{rms}$ \\
Cavity operating temperature & $2.08 \mathrm{~K}$ \\
Heat load @ $2 \mathrm{~K}$ & $<9 \mathrm{~W} / \mathrm{cavity}$ \\
Liquifier $2 \mathrm{~K}$ cooling power & $5 \mathrm{~kW}$ \\
Liquifier operating power & $5 \mathrm{MW}$ \\
\hline \hline
\end{tabular}




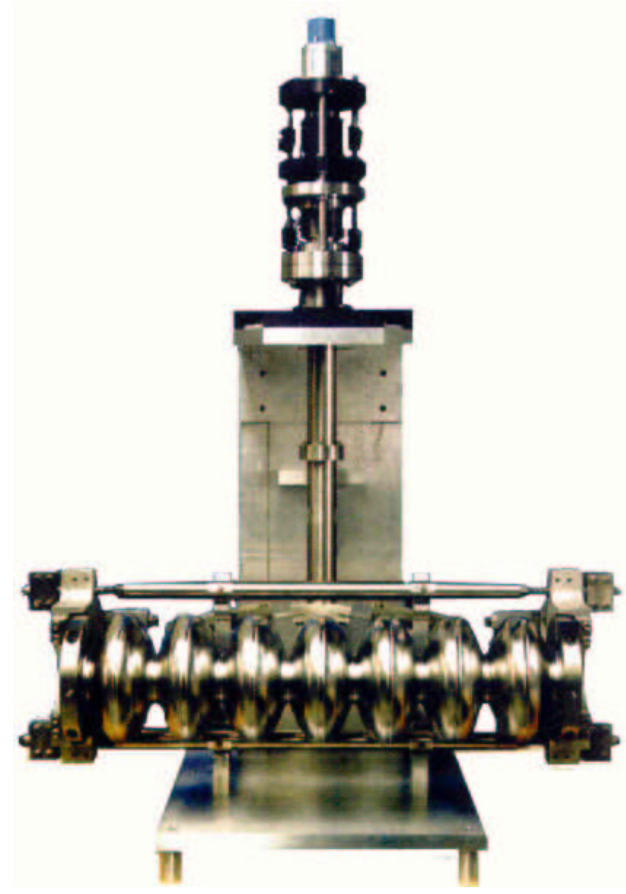

Figure 2.4: The new seven cell cavity design for CEBAF at $12 \mathrm{GeV}$. The existing cavities have a similar design but only 5 cells.

and installed in "cryounits", four of which make up an $8.25 \mathrm{~m}$ long, eight cavity "cryomodule". There is a central helium refregirator, located in the center of the racetrack shaped accelerator, that supplies $2.2 \mathrm{~K}, 2.8$ atm helium to cool down the cavities.

And last but not least, the whole accelerator operations is controlled and monitored via the EPICS control system (Experimental Physics and Industrial Control System), which enables communication between the machine operators and thousands of devices along the beam line...

\subsection{The Hall-B equipment}

A schematic view of the Hall B with the different equipments that it contains is shown in Figure 2.5 taken from reference [Mec02] of which I shall make further use in the rest of the section. Two types of experiments are routinely performed 
in the Hall B: those which use an electron beam and those which use a photon beam. The folowing subsections will essentially describe the equipment that $\mathrm{g} 7$ used, that is the standard photon setting, plus some novelties.

\subsubsection{The production and tagging of the photon beam}

This subsection is dedicated to the description of the way the photons used in the photon run experiments of Hall B are produced and "tagged" (i.e. an energy and a time information is associated to each one of them). More details can be found in [Sob00].

\section{The production of the photon beam}

As mentioned already, the CEBAF accelerator delivers an electron beam to the different Halls. The photon beam of Hall B is produced by letting this electron beam go through a thin target, the "radiator", just upstream from a magnetic spectrometer, the "tagger" and radiate. A schematic view of Hall B is given in Figure 2.6.

An electron of the original beam gets decelerated (scattered) by the electromagnetic field of a nucleus in the radiator, and in the process emits an energetic photon. The bremstrahlung radiator target ladder is based on a modification of a HARP design used throughout the CEBAF accelerator as an electron beam position monitor. Thus a standard wire scanner and four radiator targets are mounted on the same ladder assembly. This way, the position of the electron beam can be precisely determined at the exact location where the radiators are to be inserted. Radiators with high atomic numbers were used in order to minimize the effects from electron-electron bremstrahlung in the spectrum. Several thicknesses of radiator targets, ranging from $1 \times 10^{-6}$ to $1 \times 10^{-4}$ radiation lengths are available. Thinner radiators are combination films of gold on a backing of carbon, the thicker ones are free-standing gold films. 


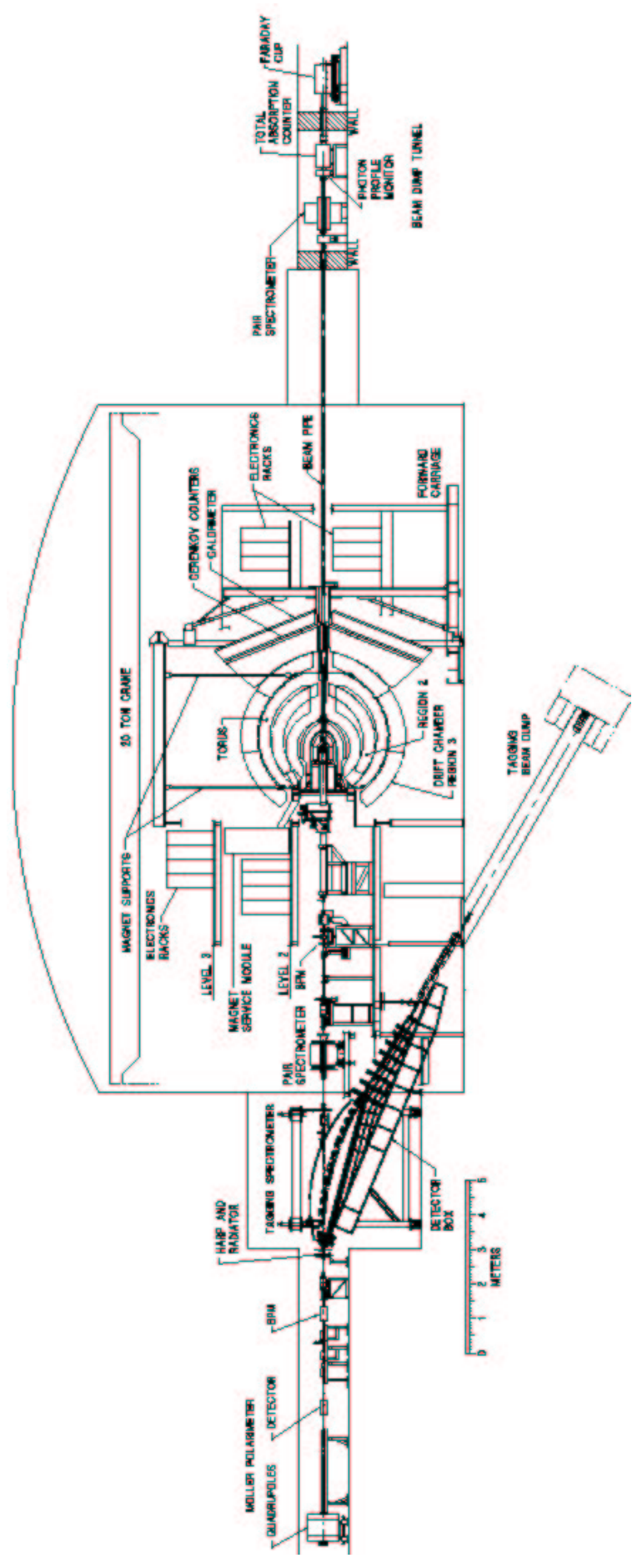

Figure 2.5: Side view of the CLAS detector in Hall B with beam line and associated equipment ([Mec02]). 


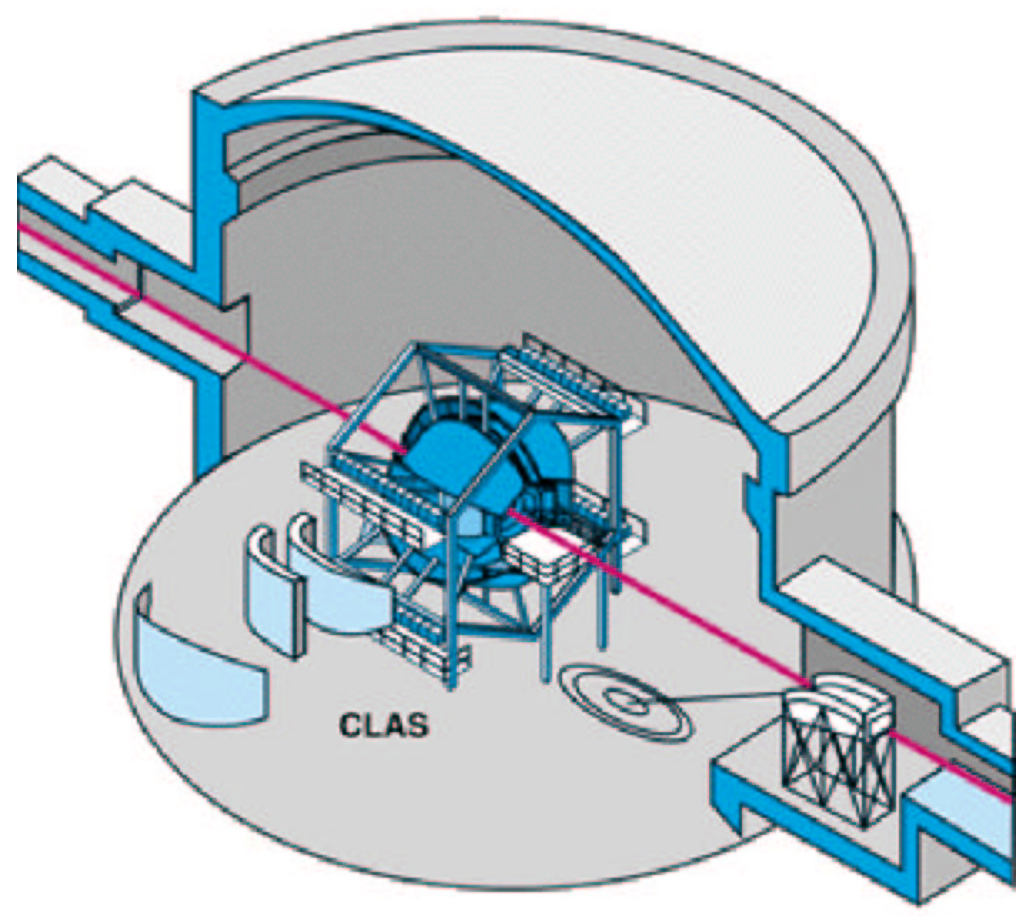

Figure 2.6: Schematic of Hall B. The beam is coming from the bottom right corner on the picture.

\section{The principle of the tagging}

The tagger is placed right after the radiator ladder along the beam line and made of a single dipole magnet combined with a hodoscope containing two planar arrays scintillators. It can detect energy-degraded electrons from a thin bremstahlung radiator and can thus tag photon energies over a range from $20 \%$ to $95 \%$ of the incident electron beam energy.

The electrons together with the photons they radiated enter the tagger, which deflects the electrons away from the beam line thanks to its dipole magnet and lets the photon beam continue straight onto the target. This is illustrated in Figure 2.7.

The energy transferred to a raditor nucleus during the deceleration (scattering) of an electron is negligibly small, so the reaction obeys the energy conservation relation

$$
E_{\gamma}=E_{0}-E_{e}
$$




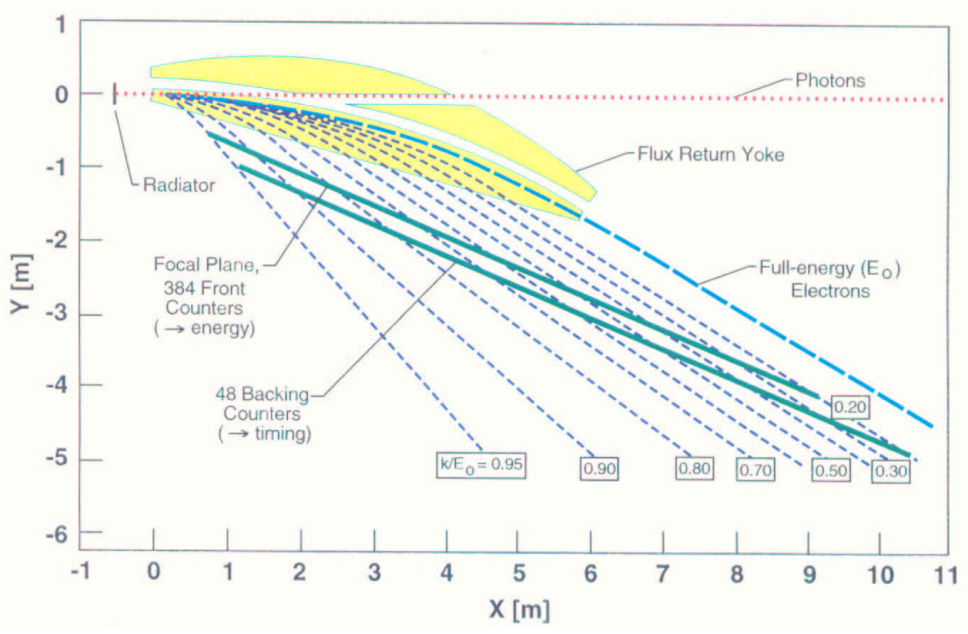

Figure 2.7: Photon beam production and tagging.

where $E_{e}$ is the energy of the outgoing electron and $E_{\gamma}$ is the energy of the emitted photon. Since $E_{0}$ is uniquely determined by the accelerator, a meassurement of the outgoing electron energy by a magnetic spectrometer thus provides a determination of the photon energy.

At energies of a few $\mathrm{MeV}$, the outgoing electron and photon emerge at very small angles relative to the incident beam direction. The angular ditribution of the photons has a characteristic angle

$$
\theta_{c}=m c^{2} / E_{0}
$$

where $\mathrm{m}$ is the electron rest mass, while the electron's characteristic angle is given by

$$
\theta_{e}=\theta_{c} E_{\gamma} / E_{e}
$$

At the Jlab energies (usually $>800 \mathrm{MeV}$ for experiments), both of these angles are of the order of $1 \mathrm{mrad}$ or smaller, so that in first approximation, both the photon and the electron are still travelling along the original beam direction at their exit from the radiator.

A collimation system, to further define the photon beam leaving the tagger, is positioned just downstream of the tagger, centered on the photon beamline. This 
system has two sets of interchangeable collimator sets inserts, with interspaced sweep magnets to clean up any charged particle background generated in the collimator walls.

\section{The tagger magnet}

The field setting of the tagger magnet is matched to the incident beam energy so that those electrons which do not radiate will follow a circular arc just inside the curved edge of the pole face, and will be directed into a shielded beam dump below the floor of the experimental hall. Electrons which do give up energy to radiate a photon, experience a greater curvature in the tagger field, and emerge from the magnet somewhere along the straight edge of the pole gap. A scintillator hodoscope along the flat focal plane downstream from this straight edge detects these energy-degraded electrons, and thereby allows for the determination of the energy of the radiated photon. This hodoscope must have adequate segmentation to deliver the desired energy resolution. Its instrumentation must also provide timing infomation sufficiently precise to allow for correlation with a particular nuclear interaction in the downstream target to within the $2 \mathrm{~ns}$ interval between beam "buckets" from the accelerator.

\section{The tagger hodoscope}

The hodoscope is made of two scintillator planes. The first plane is called the E-plane and the second plane, the T-plane, where E denotes the energy and T the time. The E-plane contains 384 narrow plastic scintillators called the E-counters to record deflected electron positions in the E-plane, an thus the momenta of those degraded electrons. The E-counters are $20 \mathrm{~cm}$ long and $4 \mathrm{~mm}$ thick, and with widths (along the dispersion direction) that range from 6 to 18 $\mathrm{mm}$ in order to subtend approximately constant momentum interval of $0.003 E_{0}$. Each E-counter optically overlaps its adjacent neighbors by one third of their respective widths, thus creating 767 separate photon energy bins through appro- 
priate recording of coincidences and anti-coincidences, and providing an energy resolution of $0.001 E_{0}$. Light from the scintillators is collected by optical fiber light guides and transmitted to photo-multiplier tubes. The T-plane provides a timing resolution better than 300 ps with its 61 T-counters, $2 \mathrm{~cm}$ thick, read out using phototubes attached by solid light guides at both ends of each scintillator. The T-counter scintillators are organized in two groups, with the first group of 19 narrower counters spanning the photon energy range from $75-95 \%$ of the incident electron energy, and the remaining group of 42 counters spanning the range from 20-75\%. The T-counter widths (along the dispersion direction) are varied to compensate for the $1 / E_{\text {gamma }}$ behaviour of the bremstrahlung cross section, so that the rate in each detector remains approximately the same within each group, with the rate in the first group approximately $1 / 3$ of that in the second group. T-counter lengths (transverse to the momentum direction) are also varied from $20 \mathrm{~cm}$ at the high electron momentum end to $9 \mathrm{~cm}$ at the low momentum end. These lengths were chosen to be large enough to accept $\sim 98 \%$ of the electrons from the radiator at $E_{0}=0.8 \mathrm{GeV}$.

\section{The tagger electronics}

As for the tagger electronics, each of the 122 T-counter photomultiplier signals is fed to one input of a fast $(200 \mathrm{MHz})$ constant-fraction discriminator (Phillips 715). The output of each fast discriminator is then sent to one of four 64-input, 12-bit, multi-hit, FASTBUS TDCs (LRS 1872A), operating in the 50 ps/channel resolution mode. The output of each E-counter photomultiplier tubes is fed to a signal-amplifier, discriminator, multiplexer and logic module (ADML). The amplifier discriminator in this module is a Phillips 6816, 16-channel, time-overthreshold unit. The output of each E-counter discriminator is fed to one input of a 96-input, 12-bit, multi-hit, FASTBUS TDC (LRS 1877), running in commomstop mode with a timing resolution of $500 \mathrm{ps} /$ channel. The stop signal is supplied by the CLAS event trigger. 
The timing information from the E- and T-counters is used during data analysis to establish the hit patterns and to make tight timing coincidences between the counters. Timing windows of less than $0.5 \mathrm{~ns}$ for the T-counters and $3 \mathrm{~ns}$ for the E-counters allow software rejection of most background events.

\subsubsection{The CLAS detector}

The CEBAF Large Acceptance Spectrometer (CLAS) has been operating for intermediate energy electromagnetic physics experiments since December 1997 ([Bro00]). The Table 2.2 (from reference [Mec02]) gives a summary of the detector's design goals. The magnetic field is given by six superconducting coils (main torus), an extra six coil torus of smaller size (mini torus) can be used to get additional field arround the target. The detector is otherwise made of 3 regions of drift chambers, time of flight scintillators, Cerenkov counters, electromagnetic calorimeters, and what's called the large angle calorimeters as can be seen in Figure 2.8. A description of these individual components and their specifications will be given in the subsections that follow.

A conventional spherical coordinate is generally used to describe CLAS. The z-axis is taken to lie along the beam direction, with $\theta$ as the polar (scattering) angle, and $\phi$ as the azimuthal angle. The $\mathrm{x}$ and $\mathrm{y}$ directions are then respectively horizontal and vertical in the plane normal to the beam.

\section{The CLAS magnetic field}

The CEBAF Large Acceptance Spectrometer design is based on a toroidal magnetic field. Primary requirements driving this choice were the ability to measure charged particles at small angles with good momentum resolution, geometrical coverage to large angles in the laboratory, and a magnetic-field-free region around the target to allow the use of polarized targets. A view of the particle detection system normal to the direction of the beam (cut in the target 
Table 2.2: Summary of the detector design goals ([Mec02]).

\begin{tabular}{|c|c|c|}
\hline Capability & Quantity & Range \\
\hline \multirow[t]{4}{*}{ Coverage } & charged particle angle & $8^{\circ} \leq \theta \leq 140^{\circ}$ \\
\hline & charged particle momentum & $0.1 \leq p \leq 4 G e V / c$ \\
\hline & photon angle & $8^{\circ} \leq \theta \leq 45^{\circ}$ \\
\hline & photon energy & $E_{\gamma} \geq 0.1 G e V$ \\
\hline \multirow[t]{6}{*}{ Resolution } & momentum $\left(\theta \leq 30^{\circ}\right)$ & $\sigma_{p} / p \approx 0.5 \%$ \\
\hline & momentum $\left(\theta \geq 30^{\circ}\right)$ & $\sigma_{p} / p \approx(1-2) \%$ \\
\hline & polar angle & $\sigma_{\theta} \approx 1 \mathrm{mrad}$ \\
\hline & azimuthal angle & $\sigma_{\phi} \approx 4 m r a d$ \\
\hline & time (charged particles) & $\sigma_{t} \approx(100-250) p s$ \\
\hline & photon energy & $\sigma_{E} / E \approx 10 \% / \sqrt{E}$ \\
\hline \multirow[t]{3}{*}{ Particle ID } & $\pi / \mathrm{K}$ separation & $p \leq 1.5 \mathrm{GeV} / \mathrm{c}$ \\
\hline & $\pi / \mathrm{p}$ separation & $p \leq 3 G e V / c$ \\
\hline & $\pi^{-}$misidentified as $e^{-}$ & $\leq 10^{-3}$ \\
\hline \multirow[t]{2}{*}{ Luminosity } & electron beam & $L \approx 10^{34} \mathrm{~cm}^{-2} \mathrm{~s}^{-1}$ \\
\hline & photon beam & $L \approx 5 \times 10^{31} \mathrm{~cm}^{-2} \mathrm{~s}^{-1}$ \\
\hline \multirow[t]{2}{*}{ Data acquisition } & event rate & $1.5 \mathrm{kHz}$ \\
\hline & data rate & $10 \mathrm{MB} / \mathrm{s}$ \\
\hline
\end{tabular}




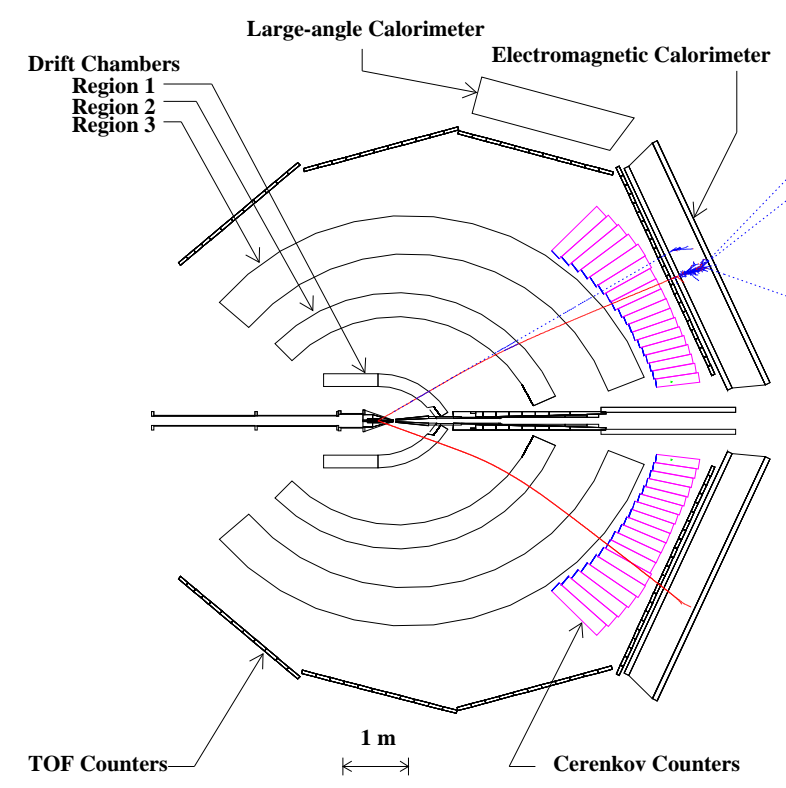

Figure 2.8: The CLAS detector, top view, cut along the beam line ([Mec02]). Typical electron, photon and proton tracks from an interaction are superimposed on the figure.

region) can be seen in Figure 2.9. One can see the localization of the main and the mini torus with respect to the rest of the detector components.

\section{The main torus}

The main torus generates the primary field for CLAS. It is always present, be it for an electron run or a photon run type of experiment, as it gives the field neccessary to do the tracking of charged particles with the drift chambers. The six superconducting coil geometry gives a field pointing primarily in the $\phi$-direction. The layout of the coils and the contours of absolute field strengths are shown in Figure 2.10. The Figure 2.11 shows the magnetic field vectors in a plane perpendicular to the torus axis at the target position. The kidney-shape of the coils results in a high field integral for forward-going particles (typically high momentum), and a lower field integral for particles emitted at larger angles. This geometry also preserves a fields-free region at the center for a polarized target. The maximum design current is $3860 \mathrm{~A}$, the total number of turns is $5 \times 10^{6}$ 


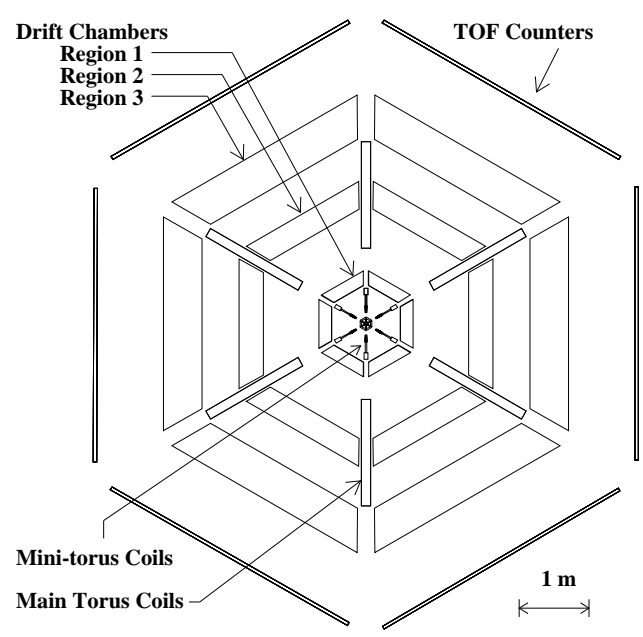

Figure 2.9: The CLAS detector, cut perpendicular to the beam line ([Mec02]).

(summed over all loops). At this current, the integral magnetic field reaches 2.5 Tesla.meter in the forward direction, dropping to 0.6 Tesla.meter at a scattering angle of 90 degrees. Routine operation has been limited to $87 \%$ of the maximum current (3375 A) to keep internal mechanical stresses within conservative limits.

Each of the six coils has 4 layers of 54 turns of aluminium-stabilized $\mathrm{NbTi} / \mathrm{Cu}$ conductor. Cooling of the coils to $4.5 \mathrm{~K}$ is accomplished by forcing supercritical helium through cooling tubes located at the edge of the windings. Superinsulation and an intermediate liquid-nitrogene cooled heat shield reduce that heat load. The coils are designed to be self-protecting in case of a quench.

\section{The mini torus}

The mini torus is typically not used for photon experiments but used for electron experiments. In this second type of experiments, the beam creates a big amount of low energy electrons by Møller interaction (electron-electron scattering) in the target. An additional magnetic field around the region I of the drift chambers, supplied by the mini torus, is necessary to bend these electrons 


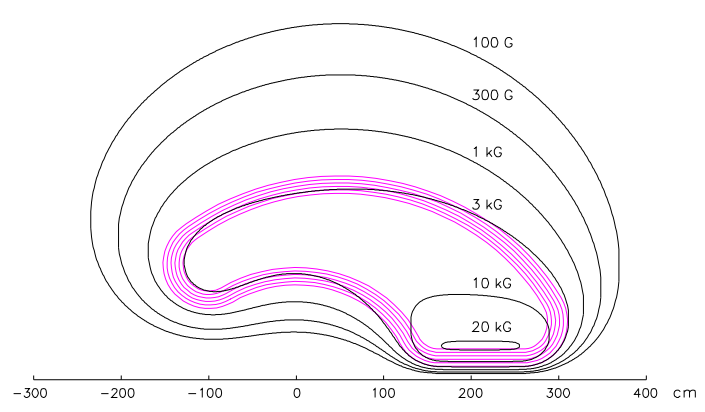

Figure 2.10: Contours of constant absolute magnetic field for the CLAS toroid in the midplane between coils $([\mathrm{Mec02}])$. The projection of the coils onto the midplane is shown for reference.

into the beam pipe. Because the g7 target had high-Z material in it, it also was a source of a big amount of low energy electron-positron pairs. This required the use of the mini torus, contrary to the typical photon experiments. Testing the efficiency of the mini torus in removing these electrons and positrons was a big part of the simulations prior to the run. This topic will be discussed in detail later on.

The mini-torus, just like the main torus, is a toroidal magnet, consisting of six resistive water cooled coils. The maximum current in the magnet is $8000 \mathrm{~A}$. Again, routine operation has been limited to $75 \%$ of the maximum current (6000 A) to keep internal mecanical stresses within reasonable limits.

\section{The Drift Chambers}

A good source of inspiration for this section was the reference [Mes00], . The reader interested in more detail about the drift chambers, should refer to it. 


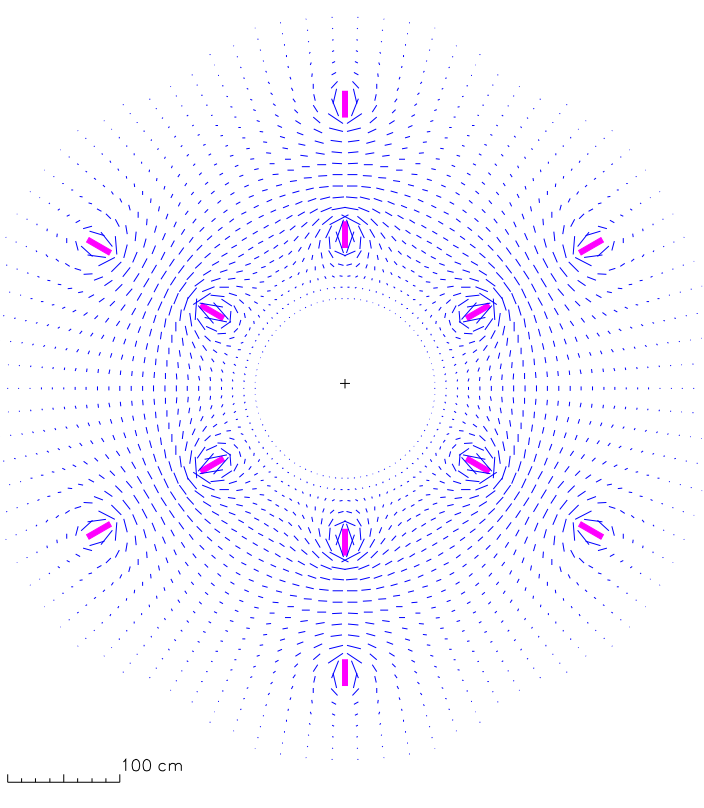

Figure 2.11: Magnetic field vectors for the CLAS toroid transverse to the beam in a plane centered on the target ([Mec02]). The length of each segment is proportional to the field strength at that point. The six coils are seen in cross section. 
CLAS was designed to track charged particles with momenta greater than $200 \mathrm{MeV} / \mathrm{c}$ over the polar angle range from $8^{\circ}$ to $142^{\circ}$, while covering up to $80 \%$ of the azimuth. In order to achieve this, it is necessary to reconstruct the trajectories of all charged particles that do not strike the cryostat of the toroidal magnet. All non-active parts of the drift-chamber structure are required to reside in the "shadow" of the torus cryostat as viewed by rays from the target, imposing tight space constraints.

The magnet coils naturally separate the detector into six independent tracking areas or "sectors", since a particle that leaves the target and enters a sector stays within that sector (indeed the CLAS toroidal magnet bends the charged particles towards or away from the beam axis but leaves the azimuthal angle essentially unchanged). To simplify the detector design and construction, 18 separate drift chambers were built and located at three radial locations in each of the six sectors. These radial locations are referred to as "Regions". The "Region One" chambers (R1) surround the target in an area of low magnetic field, the "Region Two" chambers (R2) are somewhat larger and are situated between the magnet coils in an area of high magnetic field near the maximum track sagitta, and the "Region Three" chambers (R3) are the largest devices, radially located outside of the magnet coils.

To optimally fill the wedge-shaped volume between the torus coils, the chamber bodies were designed to support wires running between two endplates, each parallel to its neighboring coil plane, and thus tilted at $60^{\circ}$ with respect to each other. This design provides maximum sensitivity to track momenta since the wire direction is approximately perpendicular to the bend plane of the curved trajectories. The wire midpoints are arranged in "layers" of concentric circles. For pattern recognition and tracking, the wire layers are grouped into two "superlayers", one axial to the magnetic field, and the other tilted at $6^{\circ}$ stereo angle around the radius of each layer to provide azimuthal information. Each superlayer nominally consists of six layers of drift cells as shown in Figure 2.12. 


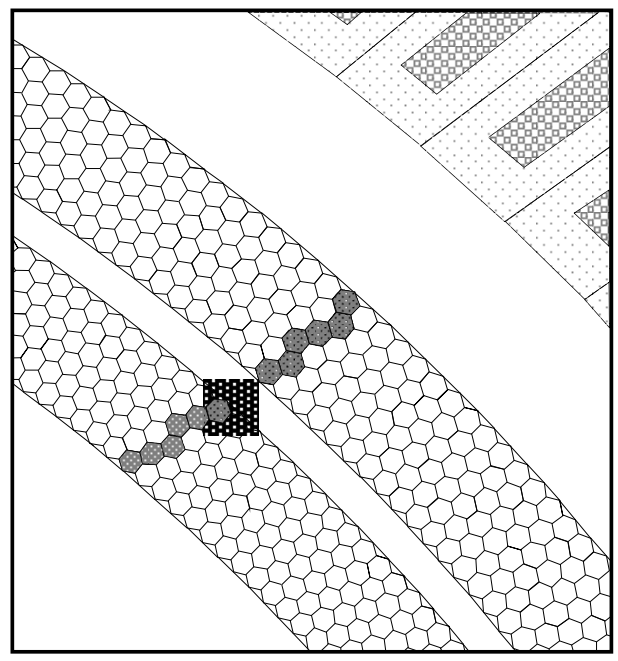

Figure 2.12: Representation of portion of a region III sector of the drift chambers showing the layout of its two superlayers ([Mes00]). The sense wires are at the center of each hexagone and the field wires are at the vertices The perimeters of the hexagones are shown to outline each cell. Not shown are the guard wires that surround each superlayer. In this view, projected on the mid-plane of the sector, a passing charged particle is shown by the highlighted drift cells that have fired. Beyond the drift chamber, in the upper right corner, the edge of a Cerenkov detector is shown. 
The wires are shifted by half the nominal position in successive layers. This "brick-wall" pattern of wires in neighboring layers, with a repeating pattern of two field-wire layers and one sense-wire layer, results in a quasi-hexagonal pattern with six field wires surrounding one sense wire. The cell size increases uniformly with increasing radial distance from the target. A layer of guard wires surrounds the perimeter of each superlayer with high-voltage potential adjusted to reproduce the electric-field configuration given by an infinite grid of hexagonal cells, i.e. the electric field at the sense wire surface is approximately the same for all sense wires, independent of wire-layer number.

The three chamber Regions share many of the same basic design elements. Each is made of six identical wedge-shaped sectors constructed from a pair of long wire-supporting endplates that bear both the load of the wire tensions and the weight of all associated hardware. A representative chamber is shown in Figure 2.13. Each endplate contains thousands of accurately positioned holes into which the wire-fixture assemblies were placed. The sense wire for all chambers consists of $20-\mu \mathrm{m}$ diameter gold-plated tungsten, the smallest practical choice. Tungsten was chosen because of its durability, and the gold-plating of the wires, amounting to a thickness of $0.127 \mu \mathrm{m}$, ensures chemical inertness as well as a smooth surface finish. The electric field at the surface of the sense wires is about $280 \mathrm{kV} / \mathrm{cm}$. The field wire for all chambers consists of $140-\mu \mathrm{m}$ diameter goldplated aluminium 5056 alloy. Aluminium was chosen because it has the longest radiation length of any practical wire material and thus minimizes multiple scattering. The main requirements for the chamber gas were that it should have reasonably low multiple scattering, allow for reasonable gas gains, have short collection times in order to reduce the random background expected from Møller electrons and target generated X-rays, and be inexpensive because of the large volume of chambers. Ultimately, a $90 \%$ argon- $10 \% \mathrm{CO}_{2}$ mixture was chosen for several reasons: the gas has a fairly high saturated drift velocity $(>4 \mathrm{~cm} / \mu \mathrm{s})$, and it has an operating voltage plateau of several hundred volts before break- 


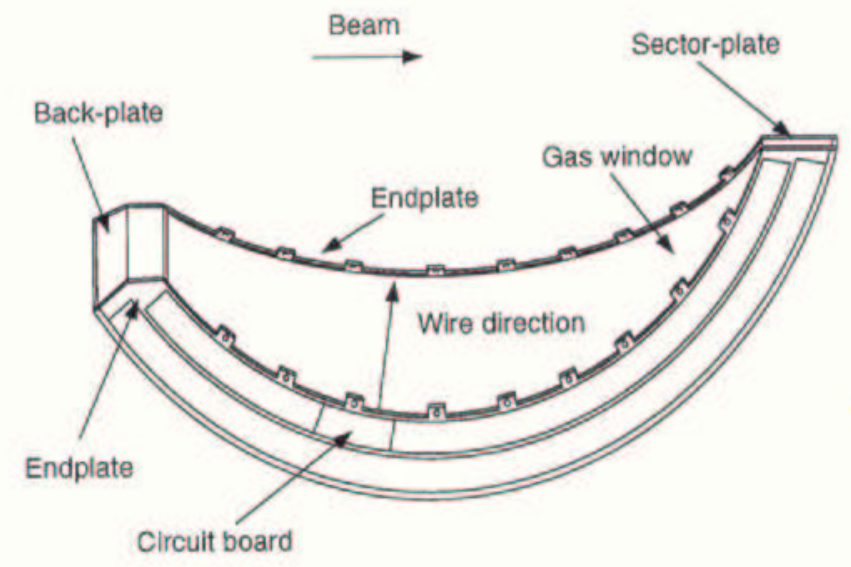

Figure 2.13: Schematic representation of a typical drift chamber sector (here an R3 sector) highlighting some of the common hardware pieces used by each $([$ Mes00]).

ing down occurs. The mixture additionaly provides good efficiency, adequate resolution, and resonable collection times. The drift-chamber frames are made primarily of aluminium (R1), fiberglass (R2), or steel-clad structural foam (R3).

R1 consists of six identical sectors integrated into a single unit. Each sector consists of 1296 hexagonal drift cells ranging in diameter from 15 to $17 \mathrm{~mm}$, with all wires located within $1 \mathrm{~m}$ of the primary beam. Each sector consists of two superlayers, the stereo layer at smaller radius. This arrangement, which is opposite to the outer two Regions, was necessitated by space constraints within the torus. While each R2 and R3 consists of 12 layers of sense wires, the limited space within the torus allowed for only 10 layers in each R1 sector. The final R1 design included 6 axial layers and four stereo layers.

Each R2 sector contains a total of 2262 hexagonal drift cells ranging in diameter from 26 to $29 \mathrm{~mm}$, with cell size increasing uniformly with wire layer number. The wires are arranged in two superlayers, one axial (at smaller radius) and one stereo (at larger radius). Each superlayer contains between 184 and 192 drift cells. One of the two endplates in each chamber has a 2.54-cm step away from the cryostat to avoid projection that decreases the azimuthal coverage slightly 
for $\theta \geq 120^{\circ}$. The hexagonal wire pattern is unaffected by this step.

The R3 chambers were designed and manufactured at Jlab. The shape of each 7-m long sector follows the outer contour of the CLAS torus. The sectors are located outside of the toroid in a field-free region at a distance from the target of between 3.0 and $3.5 \mathrm{~m}$. Each chamber has 2304 hexagonal drift cells ranging in diameter from 40 to $45 \mathrm{~mm}$, with cell size increasing uniformly with layer number. The wires are laid out with the axial superlayer closer to the target. Each of the 12 wire layers contains 192 drift cells.

The tracking resolution is the deviation of the reconstructed momenta and angles of the charged-particle tracks from their true values. Tracking uncertainties arise from three primary sources: multiple scattering in the material along the particle trajectory, geometric misalignments of the separate tracking chambers or lack of knowledge of true value of the traversed magnetic field strength, and the single-wire resolution. The single-wire resolution depends upon where within a cell the track has passed. Within a given layer, this is estimated by fitting a track to all hits except those in that layer. The residual fit is the difference between fitted distance-of-closest-approach (DOCA) of the track and the value of the DOCA calculated from the drift time in the excluded layer. The single-wire resolution worsens near the wire and also at the outer edge of the cell. This arises due to the Poisson distribution of ion-pair production along the path of the primary ion near the sense wire along with time-walk effects and the divergence of the electric field lines near the field wire. The average singlewire resolution in the mid-portion of the cell for each region is about 200 to 250 $\mu \mathrm{m}$. The whole-cell average is about 310,315 and $380 \mu \mathrm{m}$ for $\mathrm{R} 1, \mathrm{R} 2$ and R3, respectively.

\section{The Cerenkov counters}

The CLAS Cerenkov gas detector serves the function of triggering on electrons, and separating electrons from pions ([Ada01]). The detector consists of 216 
optical modules. Each module consists of three adjustable mirrors of lightweight composite construction, a Winston light collecting cone, a 5-in, photomultiplier tube, and specially designed magnetic shielding. Its design aims at maximizing the coverage in each of the six sectors up to an angle $\theta=45^{\circ}$. This is done by covering as much of the available space as possible with mirrors, and placing the light collecting cones and photomultiplier tubes (PMTs) in the regions of $\phi$ which are obscured by the magnet coils. Due to the six-fold symmetry of CLAS, the Cerenkov detectors were fabricated as six independent identical detector sectors. Each sector subtends an azimuthal anle $(\phi)$ of $60^{\circ}$ and a zenith (scattering) angle $8<\theta<45^{\circ}$. Each sector was individually assembled. Every one of the six sectors of CLAS was divided into 18 regions of $\theta$, and each $\theta$ segment was divided into two modules about the symmetry plane bisecting each sector. This results in a total of 12 identical (except for an inversion symmetry) subsectors around the $\phi$ direction for each $\theta$ interval, and a total of 216 light collection modules.

The optics of each $\theta$ module was designed to focus the light onto a PMT associated with that module and located in the region obscured by the coils. Figure 2.14 shows the optical arrangement of two modules. The array of the modules in one sector is shown in Figure 2.15. The optical elements of each module consists of two focusing mirrors, a "Winston" light collection cone, and a cylindrical mirror at the base of the cone as shown in Figure2.14. The light detection is done with by means of a 5-in. Phillips XP4500B PMT mounted at the base of the Winston cone. The trajectory of the light produced by a typical electron through the Cerenkov detector is also illustrated in Figure 2.14. Since the distance between coils increases approximately linearly with $\theta$, each of the 18 modules in $\theta$ has unique optical design parameters.

Each detector sector is then filled with $6 m^{3}$ of $C_{4} F_{10}$ (perfluorobutane) gas, which was chosen for its high index of refraction $(\mathrm{n}=1.00153)$. This results in a high photon yield, and an acceptably high pion momentum threshold of $p_{\pi} \approx 2.5 \mathrm{GeV} / \mathrm{c}$. In addition, $C_{4} F_{10}$ has excellent light transmission properties. 


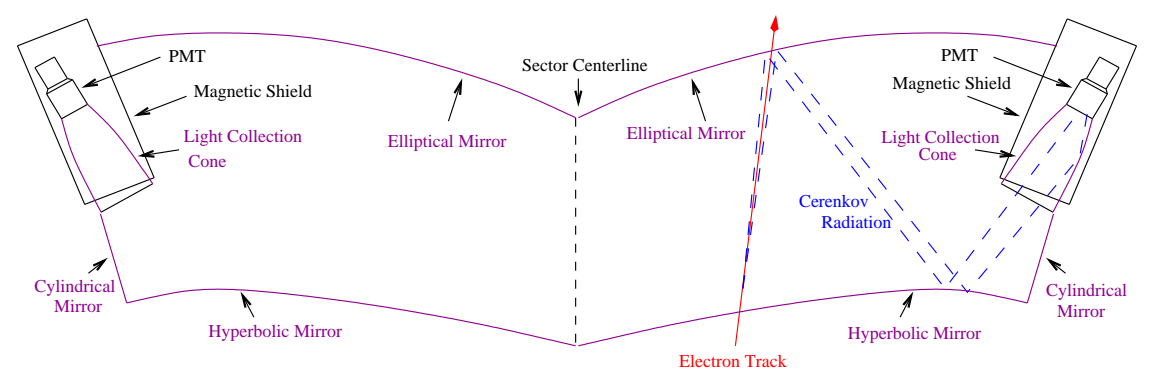

Figure 2.14: Optical arrangment of two of 216 optical modules of the CLAS Cerenkov detector, showing the optical and light collection components.

\section{Optical Mirror System}

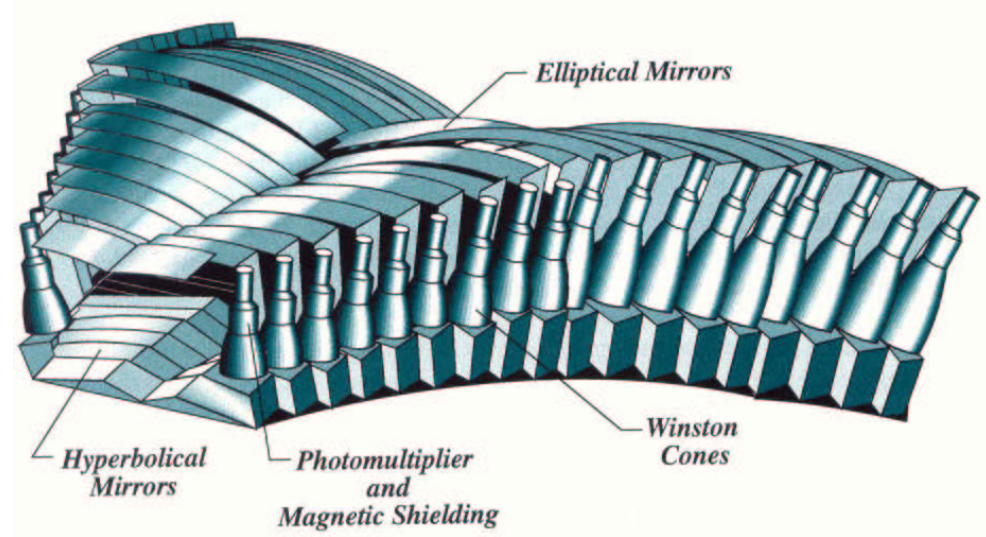

Figure 2.15: Schematic drawing of the array of optical mudules in one of the six sectors of CLAS ([Ada01]). 


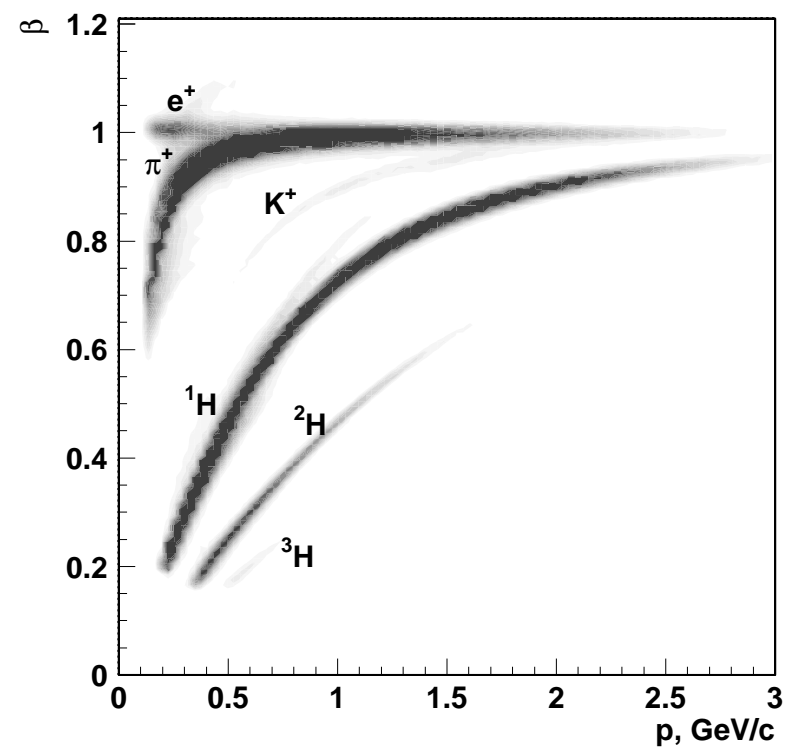

Figure 2.16: Example of $\beta / p$ plot used to do particle identification.

The measured mirror reflectivity, though typically around $90 \%$ in the visible region, drops to about $85 \%$ in the near UV, and then typically near $20 \%$ at $200 \mathrm{~nm}$, so that light absorption in gas does not play a significant role in the degradation of signal. The physical density of the gas corresponds to about 1 $\mathrm{g} / \mathrm{cm}^{2}$ effective thickness traversed by a typical particle. Except for isolated spots at the midplane, where the gaps between the mirrors are the largest, the electron efficiency within the fiducial acceptance should exceed $99 \%$.

\section{The time of flight scintillators}

Particle identification with CLAS mainly uses their time of flight (TOF) information from the time of flight scintillators in correlation with their momenta from the drift chambers (needed to plot a $\beta / \mathrm{p}$ spectrum, see example in Figure 2.16).

The TOF system covers a total area of $206 \mathrm{~m}^{2}$, representing the range from $8^{\circ}$ to $142^{\circ}$ in polar angle $\theta$ and the entire active range in azimuthal an- 


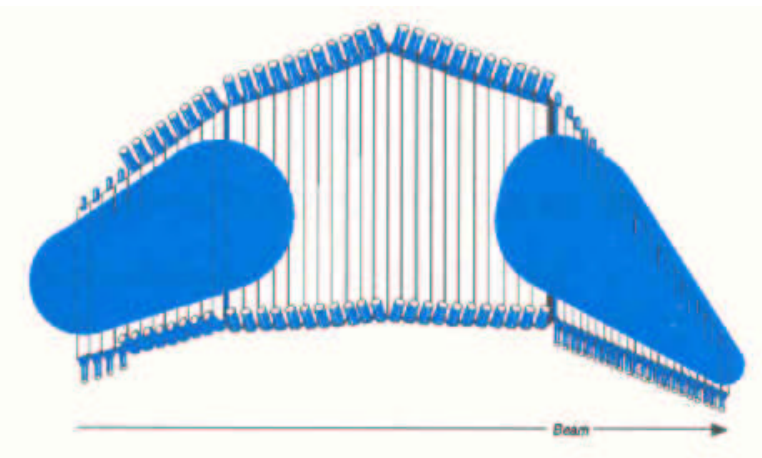

Figure 2.17: Schematic drawing of one sector of the time of flight system.

gle $\phi$ ([Smi99]). The scintillators are located radially outside the tracking system and the Cerenkov counters but in front of the calorimeters. Their alignment and relative positioning with respect to other detector subsystems can be best seen in Figure 2.8. Just like the other detectors, the TOF is divided into six sectors and each sector is made of four scintillator panels (see Figure 2.17 representing the four panels in a given sector). The scintillator thickness of $5.08 \mathrm{~cm}$ is uniform throughout, chosen to give a large signal for traversing minimum-ionizing particles. Each scintillator is placed perpendicular to the average local particle trajectory, such that the width of the counter subtends about $1.5^{\circ}$ of scattering angle. The forward counters $\left(\right.$ at $\theta<45^{\circ}$ ) are $15 \mathrm{~cm}$ wide, and the large-angle counters are $22 \mathrm{~cm}$ wide, in the $\triangle \theta$ direction. Their lengths vary from 32 to 445 cm. Thus, each sector has 57 scintillators in total with a PMT at each end.

The requirements for the TOF system include excellent timing resolution for particle identification, and good segmentation for flexible triggering and prescaling. The design parameters were chosen to allow separation of pions and kaons up to $2 \mathrm{GeV} / \mathrm{c}$. The most energetic particles are produced at small angles. The system specifications called for a time resolution of $\sigma=120 \mathrm{ps}$ at the small angle and $\sigma=250 \mathrm{ps}$ at angles above $90^{\circ}$. Particle identification is achieved by off-line analysis that combines leading-edge time measurements with pulse-height information from time-walk corrections. The TOF system is also used for energy-loss 
measurements and velocity determination in specific instances. Pulse-height information, being proportional to the energy-loss in the counter, provides an independent means for the identification of slow particles. Also the flight time can provide a more accurate measurement of particle energy than magnetic analysis for slow particles when the tracking resolution is dominated by multiple scattering. The vertex time is determined by the accelerator RF, modulo $2.004 \mathrm{~ns}$. The time of flight information from the TOF counters is essential to the identification of the right $\mathrm{RF}$ bucket for the electron run experiments, just like it is for $\mathrm{g} 7$.

\section{The Electromagnetic Calorimeters}

The last components of CLAS, those radially the farthest from its center, are the Forward Electromagnetic Calorimeter, divided into six sectors, covering the $\theta$ range up to $45^{\circ}$, and the Large-Angle Electromagnetic Calorimeter, covering only two azimuthal sectors of CLAS (corresponding to $120^{\circ}$ in $\phi$ ) and a range in the scattering angle $\theta$ between $45^{\circ}$ and $75^{\circ}$. The next two "paragraphs" give more details about each of these detector components.

\section{The Forward Electromagnetic Calorimeter}

The main functions of the forward electromagnetic calorimeter (EC) are the detection of and triggering on electrons at energies above $0.5 \mathrm{GeV}$, detection of photons at energies above $0.2 \mathrm{GeV}$ (for reconstruction of $\pi^{0}$ and $\eta$ via the measurement of their decay into 2 photons), and the detection of neutrons. Based on requirements of energy and position resolution and large acceptance coverage, a sampling calorimeter made of alternating layers of scintillator strips and lead sheets with a total thickness of 16 radiation lengths was chosen. The leadscintillator ratio is 0.24 , requiring a total thickness of $39 \mathrm{~cm}$ of scintillator and $8.4 \mathrm{~cm}$ of lead (see Figure 2.18 for a vertical section of an EC module). For more information on the EC, see the reference [Ama01].

Each EC module (representing one sector of the EC), the lead-scintillator sandwich, is contained within a volume having the shape of a nearly equilateral 


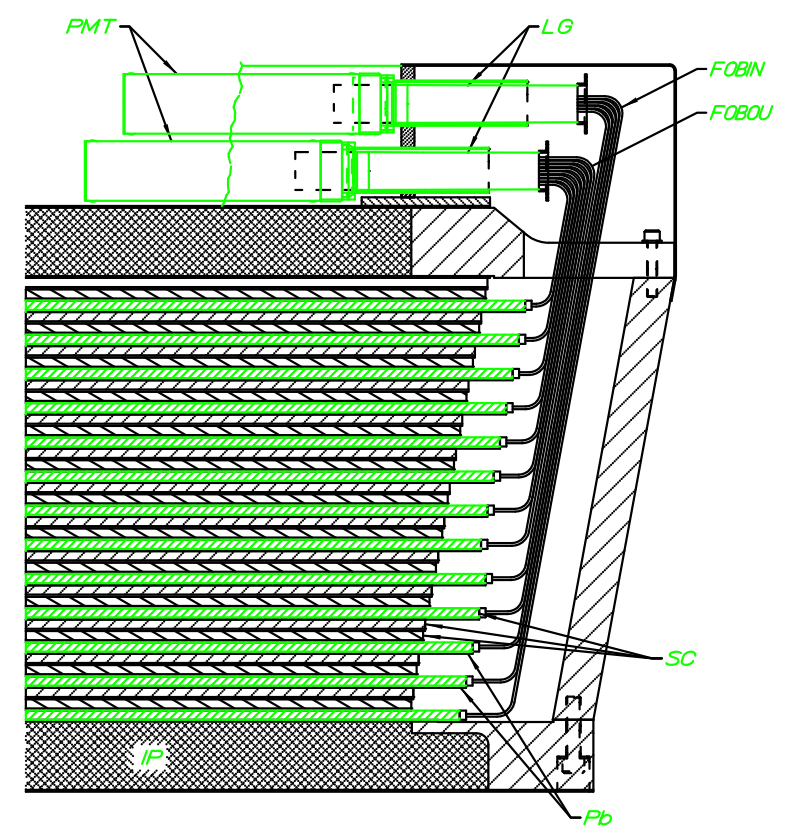

Figure 2.18: Vertical section of a module with its read-out systems: LG=Light Guide, FOBIN=Fiber Optic Bundle INner, FOBOU=Fiber Optic Bundle OUter, $\mathrm{SC}=$ scintillator, $\mathrm{Pb}=2 \mathrm{~mm}$ lead sheets, $\mathrm{IP}=$ Inner Plate.

triangle. The sandwich has 39 layers, each consisting of a 10-mm thick scintillator followed by a 2.2-mm thick lead sheet. The calorimeter utilizes a "projective" geometry pointing to the nominal target position, i.e. the area of each layer increases linearly with distance. For readout purposes, each scintillator layer consists of 36 strips parallel to one side of the triangle, with the orientation of the strips rotated by $120^{\circ}$ in successive layers (Figure 2.19). Thus, there are three orientations, or views (labeled U, V, and W), each containing 13 layers, which provide stereo information on the location of the energy deposition. Each view is further subdivided into an inner (5 layers) and an outer (8 layers) stack, to provide longitudinal sampling of the shower for improved electron/hadron separation. To reconstruct a hit in the EC, energy deposition is required in all three views $(\mathrm{U}, \mathrm{V}, \mathrm{W})$ of inner or outer layers of a module.

The energy resolution of the EC can be parametrized as

$$
\sigma / E=10.3 / \sqrt{E} \%
$$




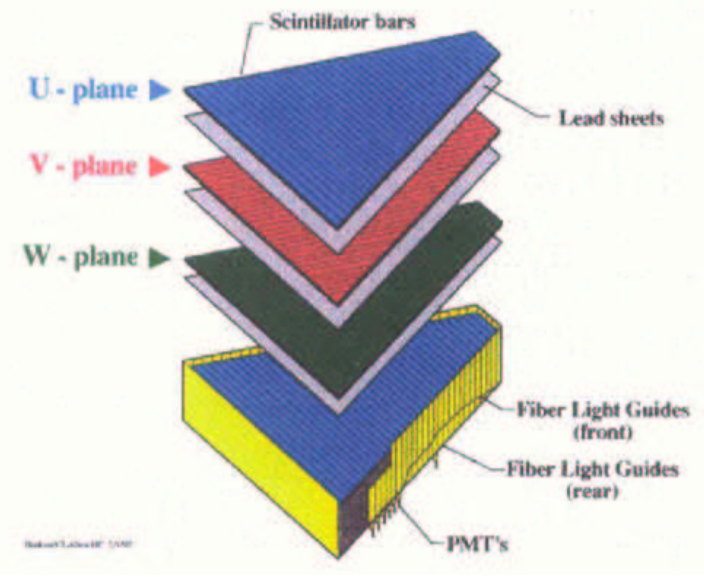

Figure 2.19: Exploded view of one of the six CLAS electromagnetic calorimeter modules ([Mec02]).

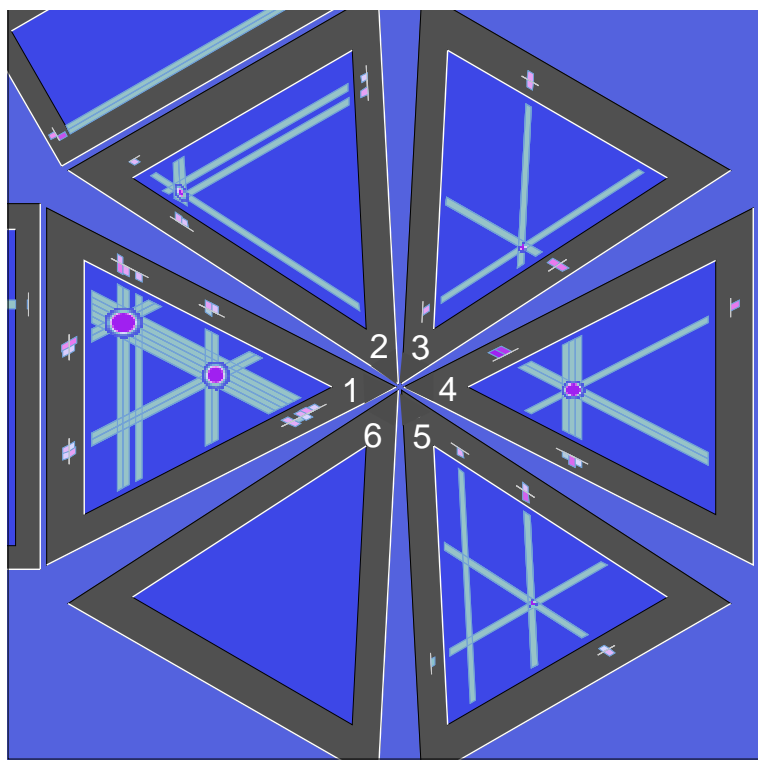

Figure 2.20: Event reconstruction in the electromagnetic calorimeter. In sectors $2,3,4$, and 5 , a single intersection of peaks on each view ( $\mathrm{U}, \mathrm{V}, \mathrm{W})$ is found, while in sector 1 , two hits are reconstructed. The size of the oval at each intersection depicts the transverse energy spread in the shower ([Mec02]). 
with a negligible constant term, where $\mathrm{E}$ is in $\mathrm{GeV}$. The sampling fraction is approximately 0.3 for electrons of $3 \mathrm{GeV}$ and greater, and for smaller energies, there is a monotonic decrease to about 0.25 for electrons of $0.5 \mathrm{GeV}$. The average rms position resolution for electrons is $2.3 \mathrm{~cm}$ for deposited energy greater than $0.5 \mathrm{GeV}$. The timing resolution for electrons averages $200 \mathrm{ps}$ over the entire detector.

\section{The Large-Angle Electromagnetic Calorimeter}

The large angle electromagnetic calorimeter (LAC) enables the detection of scattered electrons and of neutral particles such as neutrons and photons coming from radiative processes or from the decays of $\pi^{0}$ and $\eta$ mesons.

The LAC is a sampling calorimeter composed of two identical modules with a multi-layer structure of lead sheets and scintillator bars similar to the forward electromagnetic calorimeter. A single LAC module consists of 33 layers, each composed of a 20 -cm thick lead foil and a 1.5-cm thick NE110A plastic scintillator bar $([\operatorname{Ros} 96])$. The geometry is projective just like for the EC with scintillators of $10-\mathrm{cm}$ average width. Each LAC module corresponds to 12.9 radiation lengths and 1.0 hadronic absorption lengths. To avoid optical coupling, 0.2-mm thick teflon sheets separate neighboring scintillators. Scintillator bars in consecutive layers are rotated by $90^{\circ}$ to form a $40 \times 24$ matrix of roughly $10 \times 10 \mathrm{~cm}^{2}$ cells (see Figure 2.21). To improve the $e^{-} / \pi^{-}$discrimination, the LAC modules are longitudinally divided into inner (17 layers) and outer (16 layers) regions with individual light readouts.

The light produced by showers in the active material is collected into light guides at each end of a given scintillator bar and then guided into 256 PMTs per LAC module.

The LAC energy resolution for electromagnetic showers can be parametrized as:

$$
\sigma(E) / E=(7.5 \pm 0.2) / \sqrt{E} \%
$$

where $\mathrm{E}$ is the energy in $\mathrm{GeV}$. The $\pi^{-}$contamination is less than $1 \%$ at a detection 


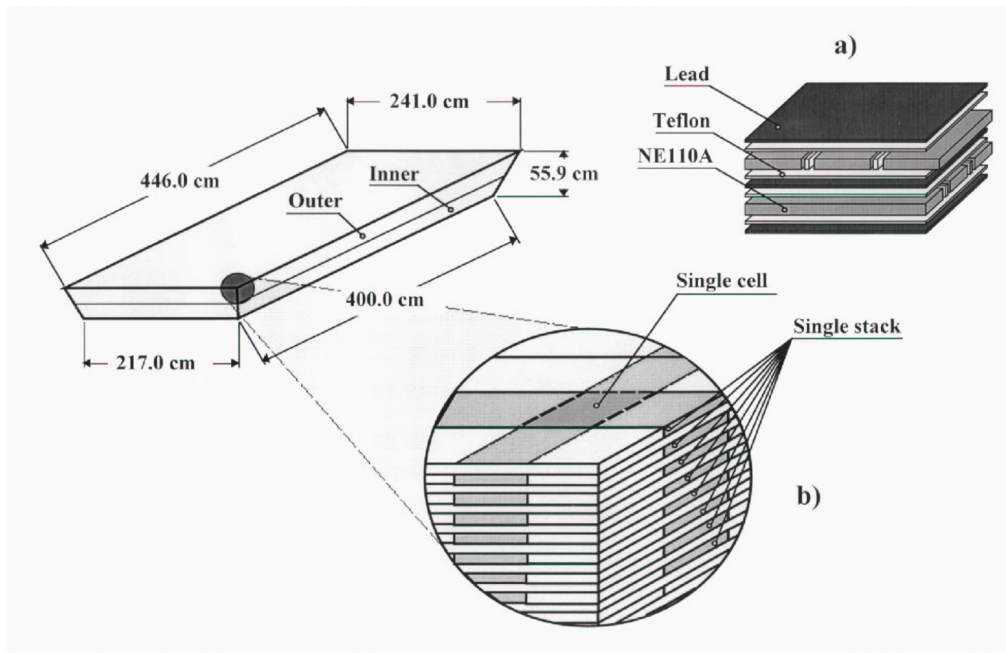

Figure 2.21: Geometry of one LAC module and a schematic view of the leadscintillator sandwich structure with details a) the calorimeter layers and b) the composition of a stack of scintillators that are read out ([Mec02]).

efficiency of $95 \%$ for $2 \mathrm{GeV}$ electrons. A good timing resolution for the $\mathrm{LAC}$ is essential to determine the momentum of neutrons via the TOF scintilators. For neutron detection, efficiencies greater than 30\%, a time resolution of 260 ps was obtained for momenta higher than $0.5 \mathrm{GeV} / \mathrm{c}$. Requiring $\beta_{\text {neutral }} \leq 0.95$ enables separation of neutrons from photons.

\section{The triggering with CLAS}

The detectors that use PMTs to generate electronic signals record signal times using LeCroy FASTBUS 1872A TDCs (Time to Digital Converters) and signal amplitudes (that give the energy deposited in each detector) using LeCroy 1881M ADCs (Analog to Digital Converters). Most TDCs are fed from leadingedge LeCroy 2313 CAMAC discriminators set at a low threshold, typically 20 $\mathrm{mV}$. The PMT signals are processed through a pipelined memory lookup by the Level 1 trigger within 90 ns. There is an additional Level 2 trigger and finally a trigger supervisor which decides to record or not a given event based on information from the Level 1 and Level 2 triggers.

The underlying idea of the Level 1 trigger is to use all available prompt 
information from the PMT channels to determine if a desired event has occured. This information includes the general location of hits in the TOF detector, the signals in the Cerenkov detector and the energy deposited in the electromagnetic calorimeter. Bit patterns from these detector subsystems are compared against patterns preloaded in memory tables for rapid response.

However, the Level 1 trigger can be set by events such as cosmic rays that lack matching particle trajectories in the drift chamber system. In order to reject triggers arizing from those events, the Level 2 trigger finds "likely tracks" in each sector, optionally performs a correlation with the Level 1 trigger, and generates a "Level 2 Fail" signal if no correlated tracks are found. While Level 1 does practically not contribute to the deadtime of the detector, Level 2 has a direct contribution to it and so the goal in designing it was to make it as fast as possible and at the same time consistent with accurately finding tracks. Identifying "likely tracks" begins by finding track segments in five superlayers in each sector (the Region I stereo superlayer is not used). Track segments are found by comparing drift chamber hits with nine templates that were designed to catch all tracks passing through a superlayer at angles of up to $60^{\circ}$. In the present implementation, a "likely track" in a sector is tagged when track segments are found in three of the five superlayers. The Level 2 trigger is satisfied by either the simple logic "OR" of these six sector tracks, or by requiring additional correlations between these tracks and the information from Level 1 .

The trigger supervisor is a custom electronics board that takes the Level 1 and Level 2 inputs from the trigger system and produces all common signals, busy gates and resets required by the detector electronics, and controls the readout.

\section{The data aquisition}

The CLAS data acquisition (DAQ) system was designed for an event rate of $1.5 \mathrm{kHz}$. During the period of CLAS commissioning in 1997, the actual rate was $400 \mathrm{~Hz}([\mathrm{Mec} 02])$. Continued development of the DAQ over five years resulted in 
routine operation at event rates between 3 and $4 \mathrm{kHz}$ for the 2000-2001 running period. At present, the limit for the total data output rate is $25 \mathrm{MByte} / \mathrm{s}$, constrained not by experimental hardware, but by the current use of the Unix file system.

The data from the different detector components are digitized in 24 FASTBUS and VME crates within the experimental hall and collected by the $24 \mathrm{VME}$ ReadOut Controllers (ROC1 to ROC24) in these crates. The arrays of digitized values associated with electronic modules are then translated into tables in which each data value (up to 16 bits) is associated with a unique identity number describing the active component within the detector.

These data arrays, or event fragments, are then buffered and sent in bunches via fast ethernet lines to the CLAS online acquisition computer (CLON10) in the control room. Three primary processes, the Event Builder (EB), the Event Transport (ET) and the event recorder (ER), comprise the main dataflow elements in the acquisition computer. The Event Builder (EB) on CLON10 assembles the incoming fragments into complete events. The individual tables are prefixed by headers to form "banks" that contain alphanumeric names that can be linked together. The completed event is then labeled by run and event number, event type, and trigger bits that are all contained in a header bank. At this stage, the event has the final format it needs for off-line analysis.

The EB passes the completed events to shared memory (ET1) on the CLAS online computer. The Event Transport (ET) system manages this shared memory, allowing access by various event producer and consumer processes on the same or remote processor systems. The Event Recorder (ER) picks up all events for permanent storage. Some events are transferred to remote ET systems for raw data checks, such as hit maps, status and events display, and for online reconstruction, analysis, and monitoring.

The ER writes the data in a single stream to magnetic media. The output files are striped accross an array of local RAID disks. A second fiber link, from 
the local RAID in the control room to the computer center a kilometer away, transfers the data files to the remote tape silo. Since the maximum tape writing speed at present is $10 \mathrm{MBytes} / \mathrm{s}$, data transfers may be started in parallel, and successive files may end up on different tapes.

\subsubsection{The photon beam flux}

The absolute photon flux is determined at very low flux rates by inserting a large lead-glass Total Absorption Shower Counter (TASC) into the photon beam. The TASC is essentially $100 \%$ efficient and can only be operated at beam currents up to $100 \mathrm{pA}$, and has to be retracted from the beam line under normal running conditions. Thus, secondary monitors, with absolute efficiency of only a few percent, but sensitive to any variation in flux, have to be cross-calibrated against the TASC at low rates and then used to monitor the flux at higher intensities.

The g7 experiment made use of the Pair Spectrometer as a beam flux monitor for its normal runs, operated with a thin conversion foil in front of it and itself situated in front of the CLAS target (it used to be situated $22 \mathrm{~m}$ behind CLAS previously). By operating the entire system in vacuum, and by using the thin pair-conversion foil that removes less than $1 \%$ of the photons from the beam, it is possible to monitor the photon flux even at high flux rates with the pair spectrometer. 


\section{Chapter 3}

\section{Simulations}

The simulation work presented in this chapter was done prior to the run of the g7 experiment in the fall of 2002, and had for goal to help choose the ideal experimental set up and conditions to carry it out. The event generators and the simulation package used to generate the events and simulate the response of the CLAS detector will be described in the appendix.

A great deal of effort had to be put in the simulations given the experimental challenges that the g7 team faced. It was the first time that nuclei heavier than carbon were used as targets in CLAS. Given the very small branching ratio for $\rho \rightarrow e^{+} e^{-}$, a very intense beam had to be sent on the target containing high$\mathrm{Z}$ material. Thus, a huge background, formed of low energy $e^{+} e^{-}$pairs, was expected around the target and the region I of the drift chambers that had to be reduced in an efficient way. In addition, because the branching ratio for $\rho \rightarrow \pi^{+} \pi^{-}$is five orders of magnitude bigger than the one for $\rho \rightarrow e^{+} e^{-}$, a very rigorous way of discriminating the electrons from the pions had to be devised, where once again the g7 team had to take up the challenge for the first time in the collaboration. In a sense, this experiment also offered an opportunity to 
explore the limits of CLAS. Its successful run profited from both the experience acquired with CLAS during the few years of running that preceded it and the rigor put in trying to understand what the ideal run conditions would be, partly using results from the pre-run simulations, partly from the test run.

\subsection{The g7 target}

Since the goal of the g7 experiment is to study the effect of nuclear density on the properties of vector mesons, the ideal target is one that contains materials with different average densities (increasing A). This was the main idea behind the design of the target.

A drawing of the target actually used for the run is given in Figure 3.1. However the target used for the simulations was slightly different. The difference between the two comes from the fact that based on the simulations, the target design was modified.

The target actually used for the experiment, contained a liquid deuterium $\left(L D_{2}\right)$ cell of $6.2 \mathrm{~cm}$ long and a diameter of about $3 \mathrm{~cm}$, and seven solid foils, each of $1.2 \mathrm{~cm}$ in diameter: carbon $(\mathrm{C})$, iron $(\mathrm{Fe})$, carbon $(\mathrm{C})$, lead $(\mathrm{Pb})$, carbon (C), titanium (Ti), carbon (C). The thicknesses of the foils were $1.5 \mathrm{~mm}, 0.6$ $\mathrm{mm}, 1.5 \mathrm{~mm}, 1 \mathrm{~mm}, 1.5 \mathrm{~mm}, 1 \mathrm{~mm}$ and $1.5 \mathrm{~mm}$, respectively. The total amount of each material was chosen to be as close as possible to $1 \mathrm{~g} / \mathrm{cm}^{2}$, according to simulations done before the 1994 proposal ([Ber94]). The distance between the $L D_{2}$ cell and the first carbon foil was chosen to be $2.5 \mathrm{~cm}$ and the spacing between each two successive solid foils was $2.5 \mathrm{~cm}$ as well.

The target used for the simulations had slight differences compared to the actual one. From left to right it contained: $L D_{2}, \mathrm{C}, \mathrm{Fe}, \mathrm{C}, \mathrm{Pb}, \mathrm{C}, \mathrm{Fe}, \mathrm{C}$, each solid foil of $2 \mathrm{~cm}$ in diameter. The $L D_{2}$ cell was $10 \mathrm{~cm}$ long and had a diameter of $4 \mathrm{~cm}$ corresponding to $1.69 \mathrm{~g} / \mathrm{cm}^{2}$ in thickness (the original idea was that, to minimize the cost and the amount of time to build the target, an existing liquid 


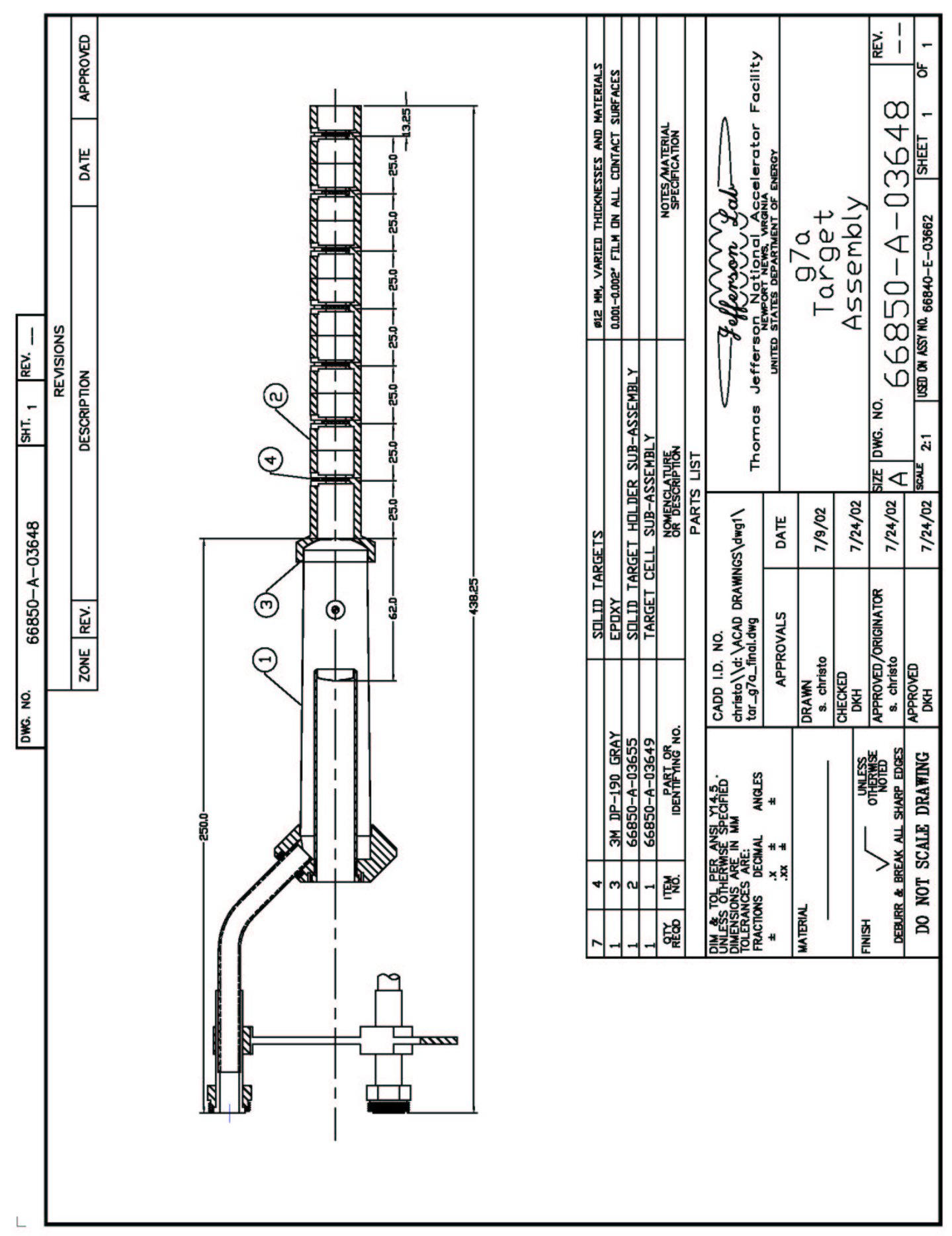

Figure 3.1: A drawing of the g7 target. The target used for the simulations was slightly different. See text for explanations. 
Table 3.1: Thickness (in "two units") of the solid g7 targets used for the simulations.

\begin{tabular}{lll}
\hline \hline Carbon & Iron & Lead \\
\hline \hline 1 g.cm & $19 . \mathrm{cm}^{-2}$ & $1 \mathrm{g.cm}$ \\
$4.4 \mathrm{~mm}$ & $1.27 \mathrm{~mm}$ & $0.88 \mathrm{~mm}$ \\
\hline \hline
\end{tabular}

cell built for the previous g2 experiment would be used. But it turned out, that both time and money were available to build a cell for g7. So, it was decided to change its dimensions to have a $1 \mathrm{~g} / \mathrm{cm}^{2}$ material). The total thickness of each of the other materials used for the simulations are given in Table 3.1. There was a distance of $2 \mathrm{~cm}$ between the liquid deuterium cell and the first carbon foil and a distance of $1.5 \mathrm{~cm}$ between each two successive solid foils.

The idea in increasing the distance separating the individual targets and decreasing their diameter as much as possible in the actual target design compared to the simulations, was to minimize the multiple scattering of the emerging $e^{+} e^{-}$ pairs (from the $\rho$ decay) inside the target. A diameter of $1.2 \mathrm{~cm}$ for the actual target was about as low as one could go given the fact the heavily collimated photon beam for $\mathrm{g} 7$, would have ended by having a diameter of $1 \mathrm{~cm}$ by the time it would have reached the target. It was essential to have the full beam on the target given the low statistics expected for the experiment. And a separation of $2.5 \mathrm{~cm}$ between the individual targets (again for the actual target) was about as wide as one could go if one wanted the target and scattering chamber to fit inside the mini torus. The smaller separation distances for the simulations were again motivated by the fact that, to minimize cost and time of construction, it was intended to use the existing e1 scattering chamber (where one had to fit in the target), but g7 finally ended up getting its own scattering chamber designed and built.

The deuterium is used as a reference. Since it is such a small nucleus, one 
expects most of the $\rho$ mesons produced on the neutron or the proton to decay outside of the nucleus. On the other hand, for the carbon, iron or lead, most of the rho should be decaying inside (especially for the lead), so that, by comparing the invariant mass spectra of the $e^{+} e^{-}$pairs from the heavy nuclei to the one from the deuterium, one might see changes to the width or peak position, if they do occur. The choice of the liquid deuterium instead of liquid hydrogen is due to a two fold argument. First, the deuterium has contributions from both the proton and the neutron, and so, has some Fermi motion inside while having almost "no nuclear density". Second, the same amount of liquid deuterium takes less space than liquid hydrogen, and lets enough space in the scattering chamber to put the other solid foils and separate them sufficiently to avoid multiple scattering effects.

\subsection{CLAS resolution for vertex reconstruction}

Being able to tell from which target a given event came from is of extreme importance to g7. This requires the spacing between the different targets to be large enough to clearly separate the deuterium events from the carbon, iron or lead events.

To have an idea of the CLAS resolution for vertex reconstruction, 20,000 events of the type $\gamma p \rightarrow \rho p \rightarrow e^{+} e^{-} p$ or $\gamma p \rightarrow \rho p \rightarrow \pi^{+} \pi^{-} p$ were generated and the number of counts for the negative outgoing particles (i.e. an $e^{-}$or a $\pi^{-}$) versus the z-position of the vertex where the event took place was plotted(see Figure 3.2). One can clearly see a distinct peak for each one of the targets. A rough gaussian fit on one of the peaks has a width of $2.44 \mathrm{~mm}$, which, indeed, is narrow enough to clearly separate the different peaks, given the fact that there is $1.5 \mathrm{~cm}$ of free space on each side of a given solid foil.

Now, one has to compare the simulations to an analysis of real data taken with CLAS. To do this, one needs a "target" used in a previous experiment that 


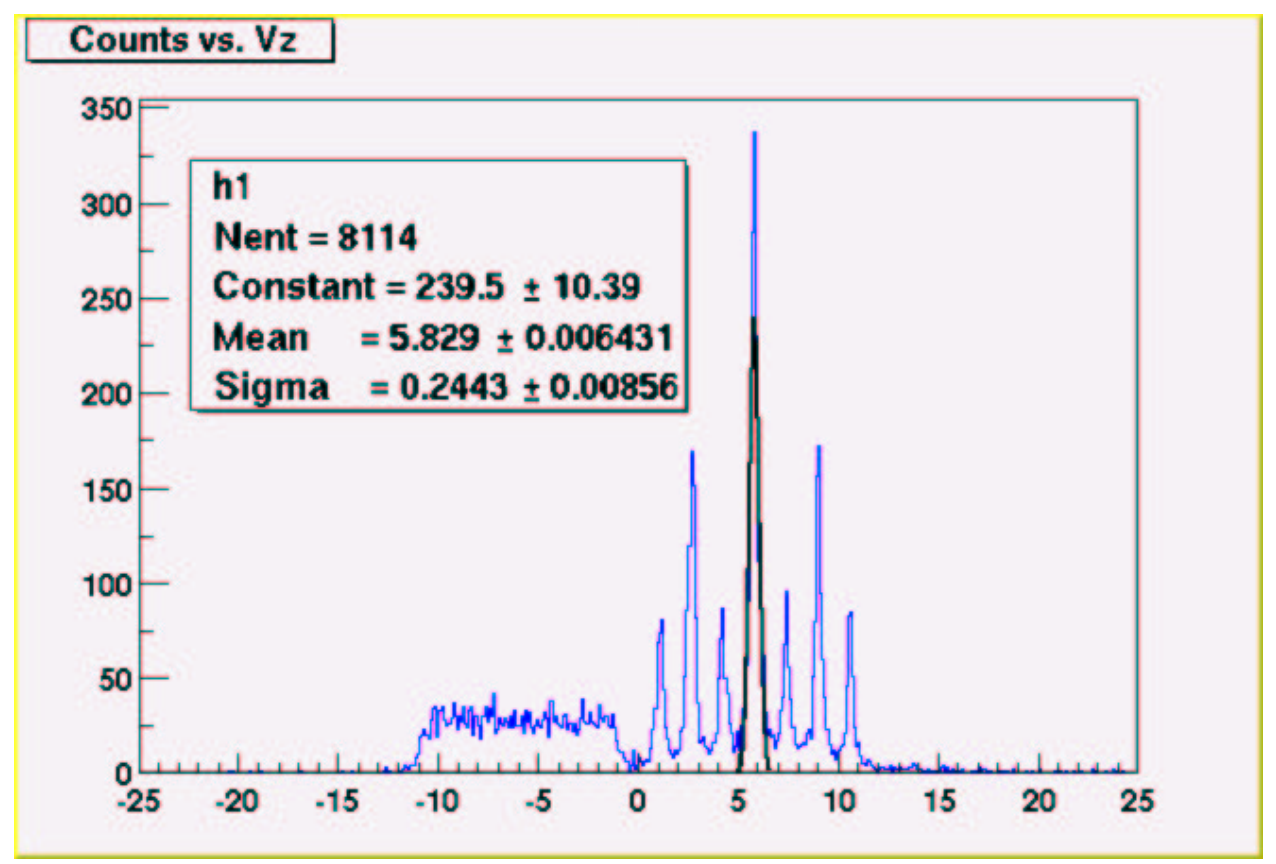

Figure 3.2: Number of $e^{-}$or $\pi^{-}$versus the z-position (in $\mathrm{cm}$ ) of the vertex for events of the type $\gamma p \rightarrow \rho p \rightarrow e^{+} e^{-} p$ or $\gamma p \rightarrow \rho p \rightarrow \pi^{+} \pi^{-} p$ with Gaussian fit. The legend gives the total number of counts and the fit parameters.

is thin enough to be comparable to one of our solid foils. An empty target run from the g1c experiment was chosen. This experiment had a cell of $18 \mathrm{~cm}$ long to contain liquid hydrogen. The measured $\mathrm{z}$ coordinate of the vertex is shown in the Figure 3.3

Around $-16 \mathrm{~cm}$, one can clearly see the super-insulation entrance window, around $-9 \mathrm{~cm}$, the actual cell's entrance window, and around $+9 \mathrm{~cm}$, the exit window (which actually is the cell's exit window plus the super-insulation layers). The thinnest wall here is the cell's entrance window $(127 \mu \mathrm{m})$, and therefore it is the one that gives the resolution.

A rough gaussian fit on the peak for the cell's exit window gives a width of $4.2 \mathrm{~mm}$, which is in "good agreement" with the simulations (one has to take into account the fact that none of the windows for g1c has the actual thickness of one of our foils). 


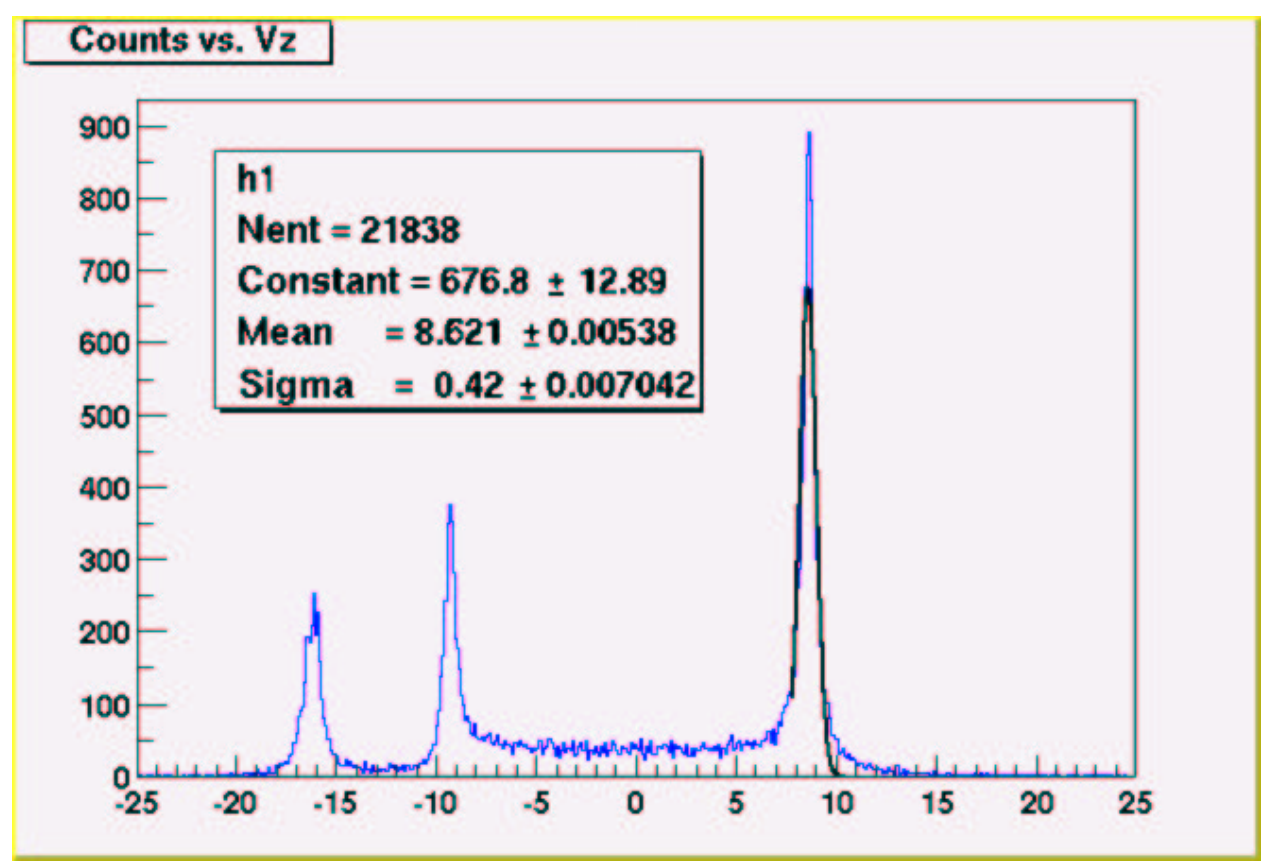

Figure 3.3: Number of outgoing $e^{-}$or $\pi^{-}$versus the z-position (in $\mathrm{cm}$ ) of the vertex for a g1c empty target run with gaussian fit. The legend gives the total number of counts and the fit parameters.

\subsection{Acceptance/Systematics}

The focus in the systematics checks is to look for any distortion of the invariant mass spectra due to the detector, or otherwise stated to see if any artificial shift in the mass of the rho or any artificial change in its width occurs, that is purely caused by the equipment used. So, the goal is to actually try to have a rough idea of our systematic errors.

For that purpose, the first thing to do is to generate events (20000 here) of the type $\gamma p \rightarrow \rho p \rightarrow e^{+} e^{-} p$, plot the invariant mass spectrum of the $e^{+} e^{-}$ pair for these "generated events" (red curve on Figure 3.4), then propagate the lepton pair in the CLAS detector, reconstruct the events, and plot once again the invariant mass spectrum of the $e^{+} e^{-}$pair for these "reconstructed events" (green curve on Figure 3.4). Then, one needs to divide the value of each bin of the "green curve" by the value of that same bin on the "red curve", and plot the values obtained in this way versus the invariant mass squared of the $e^{+} e^{-}$pair. 


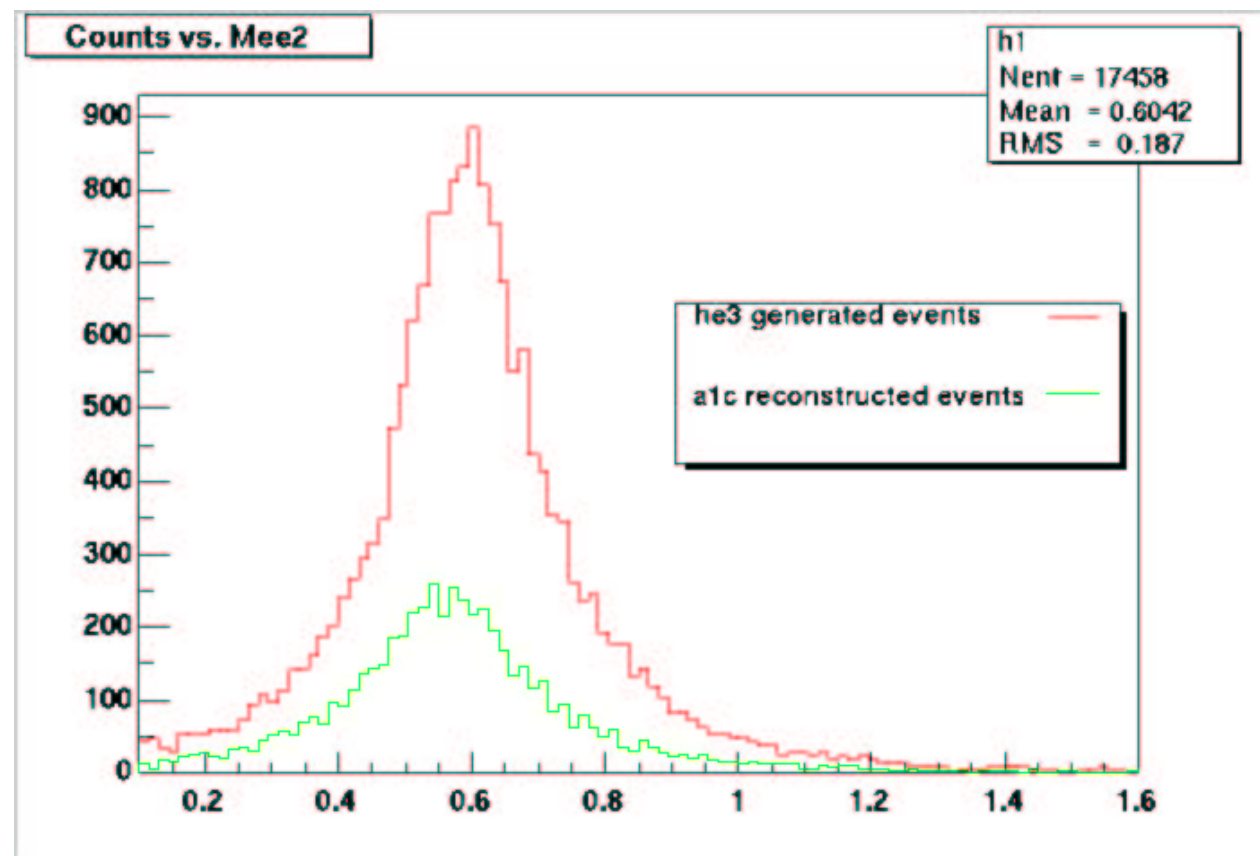

Figure 3.4: Counts versus the invariant mass squared of the $e^{+} e^{-}$pairs (in $\mathrm{GeV}^{2}$ ) for generated (red curve) and reconstructed (green curve) events of the type $\gamma p \rightarrow \rho p \rightarrow e^{+} e^{-} p$.

This gives the "acceptance plot" shown in Figure 3.5

One can see by looking at Figure 3.5 that the distribution of points does not form a flat line, but it seems that the acceptance is going up for lower masses. This is a proof that systematic errors do exist and they need to be studied and evaluated more accurately during the analysis. That the acceptance is lower for lower masses should not cause any artificial shift of the mass, but will artificially widen the width.

The second thing to do was to cut out all the "good events" in the mass spectrum and only keep the "bad events" (those that fall in the tail of the $\rho$ ) as illustrated on Figure 3.6. The peak is roughly centered at the mass squared of the proton. In fact, only the events for which the missing mass squared off of the $e^{+} e^{-}$pair (Mee2) is smaller than $0.85 \mathrm{GeV}^{2} / \mathrm{c}^{4}$ or greater than $1 \mathrm{GeV}^{2} / \mathrm{c}^{4}$ were kept. And for those bad events only, the counts versus the z-position of their vertex was plotted as shown in Figure 3.7. 


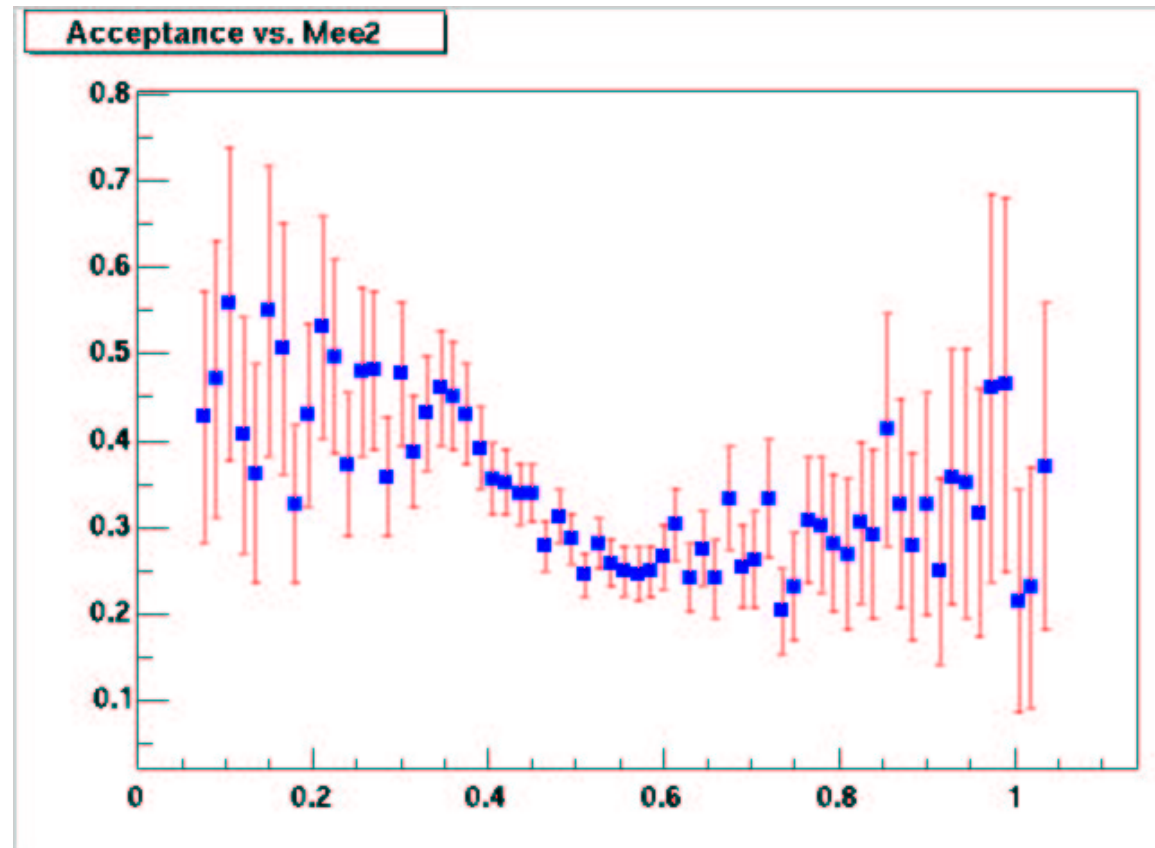

Figure 3.5: Acceptance versus the invariant mass squared of the $e^{+} e^{-}$pairs (in $\mathrm{GeV}^{2}$ ) for events of the type $\gamma p \rightarrow \rho p \rightarrow e^{+} e^{-} p$. See texte for explanations.

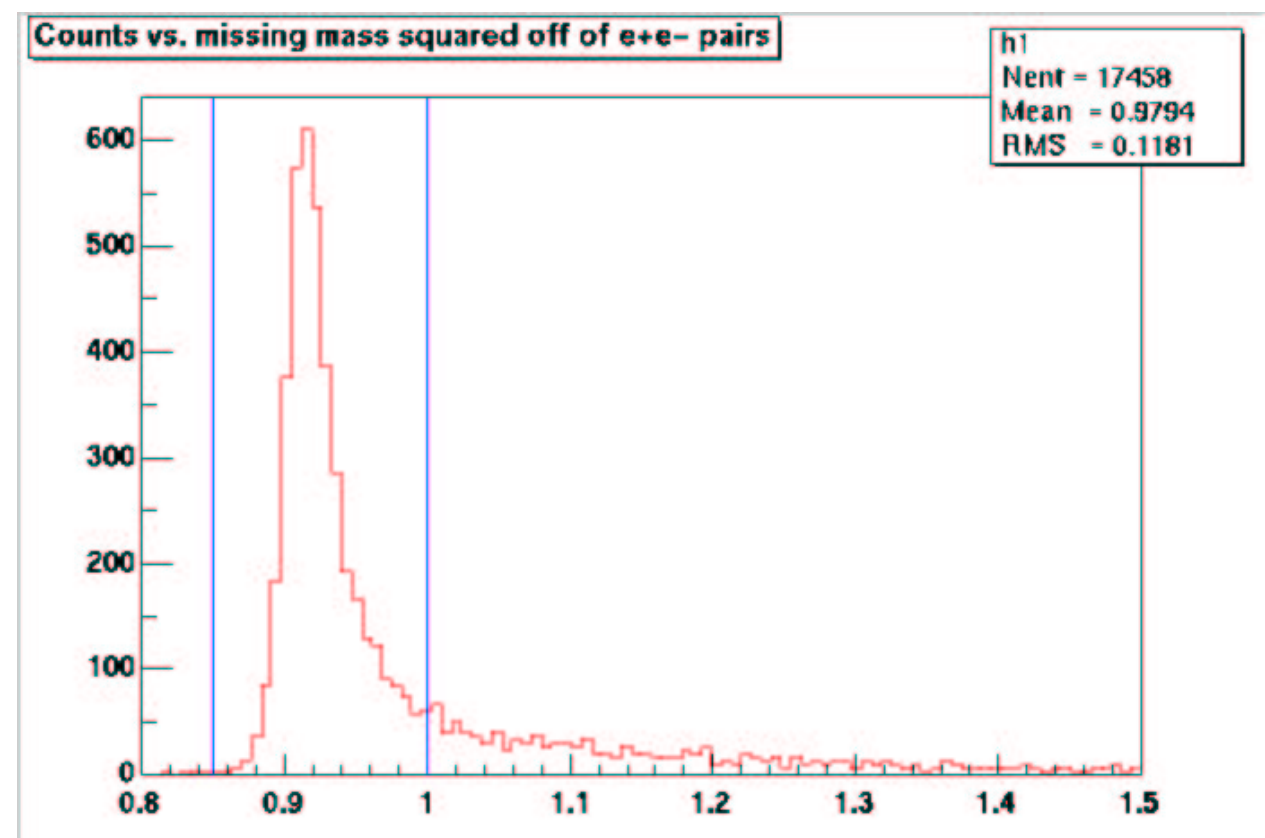

Figure 3.6: Counts versus the missing mass squared off of the $e^{+} e^{-}$pair (Mee2, in $\left.\mathrm{GeV}^{2}\right)$ with vertical lines illustrating the cut $M e e 2 \leq 0.85 \mathrm{GeV}^{2} / \mathrm{c}^{4}$ or $\mathrm{Mee} 2 \geq$ $1 G e V^{2} / c^{4}$. 


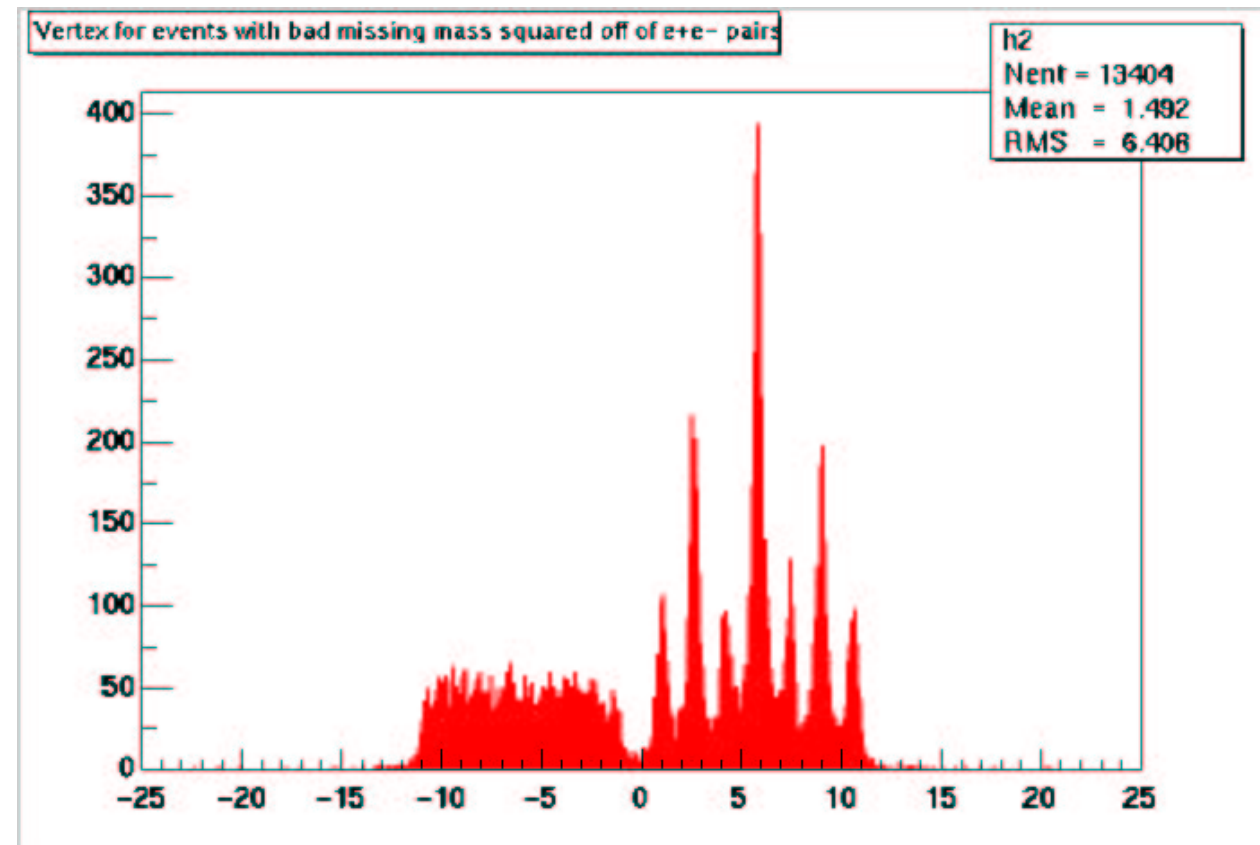

Figure 3.7: Counts versus the z-position (in $\mathrm{cm}$ ) of the vertex for the "bad events" as defined in text or illustrated in Figure 3.6.

Looking at Figure 3.7, one can see that these bad events seem to come "uniformly" from all the individual targets, or better said, there is no z-dependence in the origin of these events, or again, there is no particular z-position where these events essentially come from.

\subsection{Background}

Because the $\mathrm{g} 7$ target includes high $\mathrm{Z}$ material in it, it is a source of low energy electrons and positrons flooding the center of CLAS, in addition to the known background appearing in other photon run experiments. Simulations, of which more detail shall be given later, showed indeed that the region I of the drift chambers sees far more of these low energy electrons and positrons than the other two regions of the drift chambers. Since the goal of g7 was to run at the highest incoming photon flux possible due to the low statistics expected, it was important that the region I of the drift chambers would not limit that flux 
in any way (a high rate of low energy background electrons and positrons could saturate the region I and make them trip or simply damage them). So the goal of the simulations was to show that the mini torus field could help get rid of most of these low energy electrons and positrons, without modifying too much the event's particles trajectories, but more on that later on as well.

\subsubsection{The nature of the background}

Most of what this subsection contains can be found in the reference [Hag02]. The reader interested in more details should refer to it.

In the highest part of the bremstrahlung spectrum (a few $\mathrm{MeV}$ and up), the background is dominated by pair production (a photon converts into an $e^{+} e^{-}$ pair in the electromagnetic field of the nucleus). This is illustrated in Figure 3.9 for different nuclei.

Contributions to the photon cross section in a light element (carbon) and a heavy element (lead) are shown in Figure 3.8 At low energies, it is seen that the photoelectric effect dominates, although Compton scattering, Rayleigh scattering, and photonuclear absorption contribute as well.

It is useful to introduce the radiation length $X_{0}$ here to characterise the amount of matter traversed before the interactions mentioned like the pair production occur (more accurately $(7 / 9)$ of the mean free path for pair production by a high energy photon). $X_{0}$ is given by:

$$
X_{0}=\frac{716.4 \mathrm{gcm}^{-2} A}{Z(Z+1) \ln (287 / \sqrt{Z})}
$$

Provided some approximations, the differential cross section for pair production is given by Tsai's formula:

$$
\frac{d \sigma}{d E}=\frac{A}{X_{0} N_{A}}\left(1-\frac{4}{3} x(1-x)\right)
$$

This equation may be further integrated to obtain the high-energy limit for the 


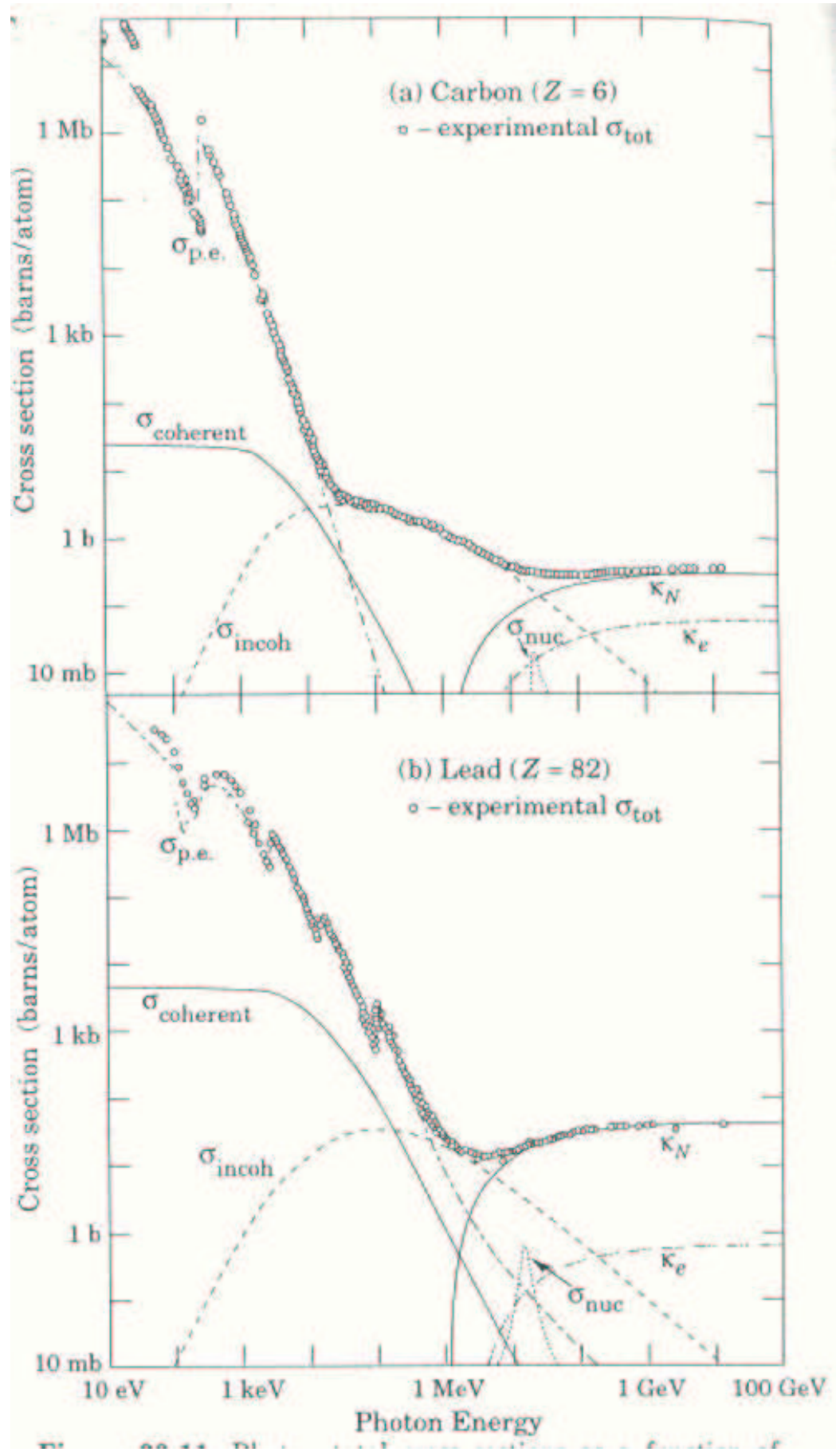

Figure 3.8: Photon total cross sections as a function of energy in carbon and lead ([Hag02]), showing the contributions of the different processes: $\sigma_{\text {p.e. }}=$ Atomic photoeffect (electron ejection, photon absorption), $\sigma_{\text {coherent }}=$ Coherent scattering (Rayleigh scattering i.e. atom neither ionized, nor excited), $\sigma_{\text {incoherent }}$ $=$ Incoherent scattering (Compton scattering off an electron), $\kappa_{n}=$ Pair production, nuclear field, $\kappa_{e}=$ Pair production, electron field, $\sigma_{n u c}=$ Photonuclear absorption (nuclear absorption, usually followed by emission of a neutron or other particle). 


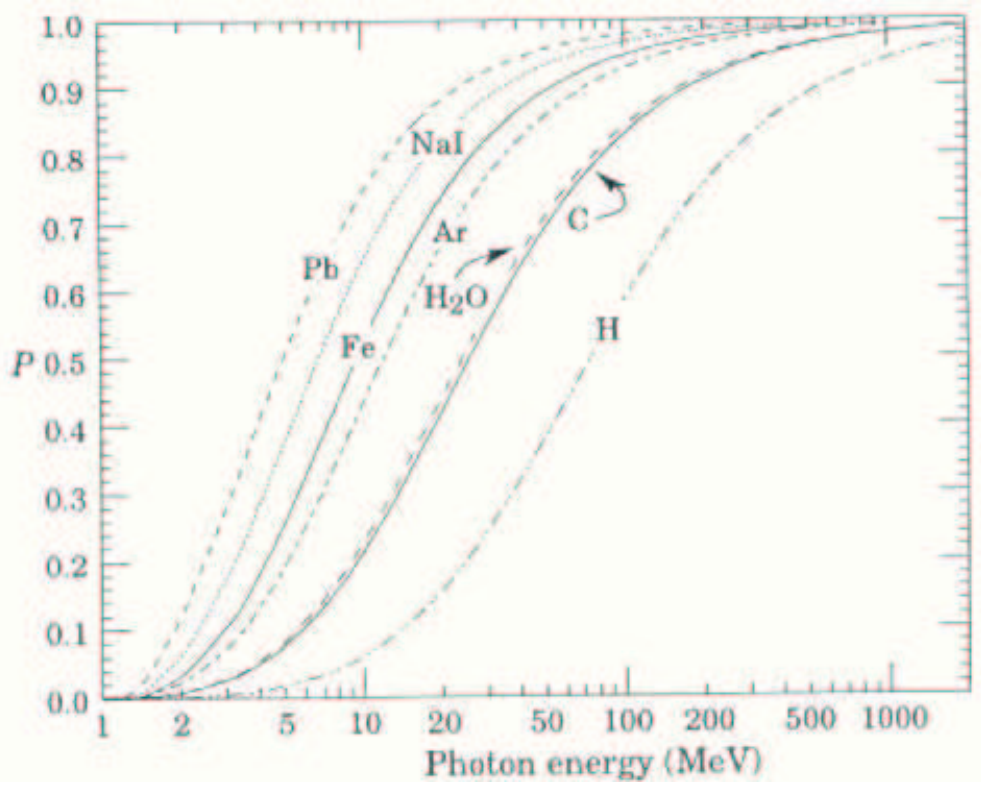

Figure 3.9: Probability $\mathrm{P}$ that a photon interaction will result in an $e^{+} e^{-}$ pair ([Hag02]). Except for a few percent contribution from photonuclear absorption around 10 or $20 \mathrm{MeV}$, essentially all other interactions in this energy range result in Compton scattering off an atomic electron.

total $e^{+} e^{-}$pair production cross section:

$$
\sigma=\frac{7}{9}\left(\frac{A}{X_{0} N_{A}}\right)
$$

The above equation is accurate to within a few percent down to energies as low as $1 \mathrm{GeV}$, particularly for high-Z materials. Thus the pair production cross section at high energies is proportional to $1 / X_{0}$ and so increases as $Z^{2} / \mathrm{A}$ as $\mathrm{Z}$ increases.

\subsubsection{The background simulations}

To simulate the low energy electron and positron background, $10^{5}$ bremstrahlung photons were generated from an initial electron beam of energy set to $2.4 \mathrm{GeV}$, distributed in a disc of $1 \mathrm{~cm}$ in diameter in the $\mathrm{x}-\mathrm{y}$ plane, and whose momentum was set parallel to the z-axis (along the beam pipe). Three different energy ranges were chosen for the photon beam:

- $100 \mathrm{MeV}-2.4 \mathrm{GeV}$ 
Table 3.2: Number of hits in the drift chambers per sector and per region for $10^{5}$ bremstrahlung photons hitting the g7 target with energies ranging from 100 $\mathrm{MeV}$ to $2.4 \mathrm{GeV}$. Case with $\mathrm{NO}$ mini torus in.

\begin{tabular}{llll}
\hline \hline & Region 1 & Region 2 & Region 3 \\
\hline \hline Sector 1 & 14671 & 1046 & 450 \\
Sector 2 & 14675 & 668 & 503 \\
Sector 3 & 15212 & 958 & 614 \\
Sector 4 & 14058 & 283 & 306 \\
Sector 5 & 14195 & 884 & 421 \\
Sector 6 & 13809 & 1572 & 573 \\
\hline \hline
\end{tabular}

$-1 \mathrm{MeV}-2.4 \mathrm{GeV}$

- $10 \mathrm{keV}-2.4 \mathrm{GeV}$

For each energy range, the corresponding $10^{5}$ photons were sent on the g7 target and the number of hits in the drift chambers per sector and per region were obtained, first in the case were the mini torus was out, then, in the case were the mini torus was in, with its current set to $-75 \%,-50 \%,-25 \%, 25 \%, 50 \%$ and $75 \%$ of its maximum value (- $75 \%$ actually means $75 \%$ of the maximum value of the current to produce a reverse magnetic field). The results are tabulated in the Tables 3.2 through 3.13 .

By looking at these values, several remarks are due. First, the mini torus does seem to reduce the background and seems to be more efficient to remove the background coming from the low energy part of the bremstrahlung photon beam. Second, it seems that the stronger the mini torus field is, the better the reduction of the background hits. Thus the best results were obtained for the case of $75 \%$ of the maximum mini torus current. There seems to be a reduction of about a factor of 3 in the case of $75 \%$ of mini torus maximum current compared 
Table 3.3: Number of hits in the drift chambers per sector and per region for $10^{5}$ bremstrahlung photons hitting the g7 target with energies ranging from 100 $\mathrm{MeV}$ to $2.4 \mathrm{GeV}$. Left:case with $25 \%$ of the maximum mini torus curent, Right: case with $-25 \%$.

\begin{tabular}{llll|lll}
\hline \hline & Region 1 & Region 2 & Region 3 & Region 1 & Region 2 & Region 3 \\
\hline \hline Sector 1 & 7517 & 562 & 1105 & 7302 & 1484 & 885 \\
Sector 2 & 8364 & 826 & 546 & 7956 & 2053 & 654 \\
Sector 3 & 9019 & 1191 & 403 & 8764 & 1329 & 629 \\
Sector 4 & 7507 & 697 & 309 & 6948 & 1199 & 688 \\
Sector 5 & 8359 & 1372 & 482 & 7472 & 1336 & 505 \\
Sector 6 & 8172 & 1794 & 335 & 7192 & 1755 & 541 \\
\hline \hline
\end{tabular}

Table 3.4: Number of hits in the drift chambers per sector and per region for $10^{5}$ bremstrahlung photons hitting the g7 target with energies ranging from 100 $\mathrm{MeV}$ to $2.4 \mathrm{GeV}$. Left:case with $50 \%$ of the maximum mini torus curent, Right: case with $-50 \%$.

\begin{tabular}{llll|lll}
\hline \hline & Region 1 & Region 2 & Region 3 & Region 1 & Region 2 & Region 3 \\
\hline \hline Sector 1 & 8625 & 1011 & 540 & 6976 & 3819 & 704 \\
Sector 2 & 8074 & 698 & 542 & 7806 & 3471 & 515 \\
Sector 3 & 8402 & 1010 & 474 & 8400 & 6333 & 416 \\
Sector 4 & 7492 & 988 & 377 & 7888 & 3715 & 485 \\
Sector 5 & 7458 & 943 & 480 & 6777 & 2178 & 361 \\
Sector 6 & 7969 & 1388 & 832 & 7756 & 4236 & 651 \\
\hline \hline
\end{tabular}


Table 3.5: Number of hits in the drift chambers per sector and per region for $10^{5}$ bremstrahlung photons hitting the g7 target with energies ranging from 100 $\mathrm{MeV}$ to $2.4 \mathrm{GeV}$. Left:case with $75 \%$ of the maximum mini torus curent, Right: case with $-75 \%$.

\begin{tabular}{llll|lll}
\hline \hline & Region 1 & Region 2 & Region 3 & Region 1 & Region 2 & Region 3 \\
\hline \hline Sector 1 & 8346 & 2434 & 743 & 8504 & 7353 & 918 \\
Sector 2 & 9269 & 1648 & 702 & 9511 & 8802 & 1049 \\
Sector 3 & 10009 & 3020 & 603 & 9106 & 8528 & 878 \\
Sector 4 & 9149 & 1984 & 1057 & 7719 & 7564 & 974 \\
Sector 5 & 8772 & 1426 & 526 & 8951 & 7357 & 1533 \\
Sector 6 & 8165 & 1406 & 695 & 8387 & 8026 & 801 \\
\hline \hline
\end{tabular}

Table 3.6: Number of hits in the drift chambers per sector and per region for $10^{5}$ bremstrahlung photons hitting the g7 target with energies ranging from $1 \mathrm{MeV}$ to $2.4 \mathrm{GeV}$. Case with NO mini torus in.

\begin{tabular}{llll}
\hline \hline & Region 1 & Region 2 & Region 3 \\
\hline \hline Sector 1 & 25859 & 883 & 428 \\
Sector 2 & 25352 & 964 & 300 \\
Sector 3 & 27722 & 793 & 152 \\
Sector 4 & 27550 & 2103 & 437 \\
Sector 5 & 25753 & 1463 & 307 \\
Sector 6 & 24821 & 1031 & 292 \\
\hline \hline
\end{tabular}


Table 3.7: Number of hits in the drift chambers per sector and per region for $10^{5}$ bremstrahlung photons hitting the g7 target with energies ranging from $1 \mathrm{MeV}$ to $2.4 \mathrm{GeV}$. Left:case with $25 \%$ of the maximum mini torus curent, Right: case with $-25 \%$.

\begin{tabular}{llll|lll}
\hline \hline & Region 1 & Region 2 & Region 3 & Region 1 & Region 2 & Region 3 \\
\hline \hline Sector 1 & 10041 & 193 & 204 & 12118 & 1492 & 327 \\
Sector 2 & 10575 & 307 & 339 & 12980 & 1366 & 520 \\
Sector 3 & 11696 & 335 & 462 & 12971 & 1873 & 190 \\
Sector 4 & 10071 & 568 & 244 & 12145 & 2256 & 605 \\
Sector 5 & 9980 & 308 & 342 & 12319 & 3001 & 345 \\
Sector 6 & 9722 & 360 & 274 & 11352 & 637 & 216 \\
\hline \hline
\end{tabular}

Table 3.8: Number of hits in the drift chambers per sector and per region for $10^{5}$ bremstrahlung photons hitting the g7 target with energies ranging from $1 \mathrm{MeV}$ to $2.4 \mathrm{GeV}$. Left:case with $50 \%$ of the maximum mini torus curent, Right: case with $-50 \%$.

\begin{tabular}{llll|lll}
\hline \hline & Region 1 & Region 2 & Region 3 & Region 1 & Region 2 & Region 3 \\
\hline \hline Sector 1 & 8113 & 621 & 340 & 10098 & 6647 & 445 \\
Sector 2 & 8378 & 1082 & 493 & 10612 & 3804 & 305 \\
Sector 3 & 8988 & 338 & 217 & 10634 & 5726 & 434 \\
Sector 4 & 8357 & 597 & 128 & 10529 & 3931 & 673 \\
Sector 5 & 7722 & 866 & 334 & 10138 & 7113 & 539 \\
Sector 6 & 8083 & 489 & 527 & 10157 & 2944 & 327 \\
\hline \hline
\end{tabular}


Table 3.9: Number of hits in the drift chambers per sector and per region for $10^{5}$ bremstrahlung photons hitting the g7 target with energies ranging from $1 \mathrm{MeV}$ to $2.4 \mathrm{GeV}$. Left:case with $75 \%$ of the maximum mini torus curent, Right: case with $-75 \%$.

\begin{tabular}{llll|lll}
\hline \hline & Region 1 & Region 2 & Region 3 & Region 1 & Region 2 & Region 3 \\
\hline \hline Sector 1 & 7139 & 1014 & 369 & 8802 & 7573 & 532 \\
Sector 2 & 8442 & 1446 & 213 & 10007 & 10993 & 578 \\
Sector 3 & 8239 & 588 & 258 & 10671 & 13408 & 706 \\
Sector 4 & 7711 & 1034 & 431 & 10129 & 12184 & 727 \\
Sector 5 & 8032 & 678 & 390 & 8551 & 12509 & 344 \\
Sector 6 & 7128 & 1326 & 316 & 8479 & 7508 & 418 \\
\hline \hline
\end{tabular}

Table 3.10: Number of hits in the drift chambers per sector and per region for $10^{5}$ bremstrahlung photons hitting the $\mathrm{g} 7$ target with energies ranging from 10 $\mathrm{keV}$ to $2.4 \mathrm{GeV}$. Case with $\mathrm{NO}$ mini torus in.

\begin{tabular}{llll}
\hline \hline & Region 1 & Region 2 & Region 3 \\
\hline \hline Sector 1 & 16566 & 987 & 147 \\
Sector 2 & 17720 & 686 & 123 \\
Sector 3 & 17671 & 686 & 356 \\
Sector 4 & 17644 & 707 & 142 \\
Sector 5 & 17537 & 539 & 22 \\
Sector 6 & 15726 & 722 & 178 \\
\hline \hline
\end{tabular}


Table 3.11: Number of hits in the drift chambers per sector and per region for $10^{5}$ bremstrahlung photons hitting the g7 target with energies ranging from 10 $\mathrm{keV}$ to $2.4 \mathrm{GeV}$. Left:case with $25 \%$ of the maximum mini torus curent, Right: case with $-25 \%$.

\begin{tabular}{llll|lll}
\hline \hline & Region 1 & Region 2 & Region 3 & Region 1 & Region 2 & Region 3 \\
\hline \hline Sector 1 & 5741 & 677 & 112 & 7740 & 1285 & 113 \\
Sector 2 & 6439 & 877 & 456 & 8630 & 2043 & 215 \\
Sector 3 & 6251 & 360 & 214 & 8028 & 1370 & 132 \\
Sector 4 & 6355 & 202 & 286 & 8774 & 743 & 122 \\
Sector 5 & 6001 & 371 & 162 & 8359 & 1526 & 279 \\
Sector 6 & 5553 & 430 & 245 & 6767 & 1582 & 144 \\
\hline \hline
\end{tabular}

Table 3.12: Number of hits in the drift chambers per sector and per region for $10^{5}$ bremstrahlung photons hitting the g7 target with energies ranging from 10 $\mathrm{keV}$ to $2.4 \mathrm{GeV}$. Left:case with $50 \%$ of the maximum mini torus curent, Right: case with $-50 \%$.

\begin{tabular}{llll|lll}
\hline \hline & Region 1 & Region 2 & Region 3 & Region 1 & Region 2 & Region 3 \\
\hline \hline Sector 1 & 5324 & 500 & 279 & 7379 & 3485 & 455 \\
Sector 2 & 5129 & 722 & 174 & 7286 & 1839 & 380 \\
Sector 3 & 5527 & 717 & 238 & 7369 & 7353 & 301 \\
Sector 4 & 4764 & 300 & 152 & 6541 & 2832 & 599 \\
Sector 5 & 6082 & 566 & 136 & 5949 & 4741 & 184 \\
Sector 6 & 5218 & 579 & 378 & 6122 & 4364 & 226 \\
\hline \hline
\end{tabular}


Table 3.13: Number of hits in the drift chambers per sector and per region for $10^{5}$ bremstrahlung photons hitting the g7 target with energies ranging from 10 $\mathrm{keV}$ to $2.4 \mathrm{GeV}$. Left:case with $75 \%$ of the maximum mini torus curent, Right: case with $-75 \%$.

\begin{tabular}{llll|lll}
\hline \hline & Region 1 & Region 2 & Region 3 & Region 1 & Region 2 & Region 3 \\
\hline \hline Sector 1 & 4998 & 447 & 137 & 5185 & 5575 & 759 \\
Sector 2 & 4764 & 514 & 223 & 6381 & 6363 & 293 \\
Sector 3 & 5290 & 571 & 315 & 6911 & 6619 & 443 \\
Sector 4 & 4763 & 354 & 173 & 6835 & 4186 & 317 \\
Sector 5 & 4703 & 398 & 385 & 6153 & 4955 & 337 \\
Sector 6 & 5071 & 668 & 357 & 5507 & 4512 & 359 \\
\hline \hline
\end{tabular}

to the case with no mini torus. And finally, the reverse magnetic field does not seem any good: it seems that $75 \%$ and $-75 \%$ give pretty much the same result for the region I of the chambers, but $-75 \%$ seems to produce a lot more hits in the region II than does $75 \%$. The origin of this disymmetry between the normal and reverse magnetic field was never really clearly understood (it may be due to the fact that the CLAS acceptance itself is different for $e^{+}$and $e^{-}$for instance, but this is pure speculation).

To illustrate this discussion and give a more visual feeling for it, pictures were drawn showing what is going on in CLAS around the target area for the different case scenarios, and are given in the Figures 3.10 through 3.17. All pictures are drawn for 1000 photons in the indicated energy range sent on the g7 target.

To comment a little bit on these pictures, the plots for the $1 \mathrm{MeV}-2.4 \mathrm{GeV}$ energy range were drawn, then this range was split into 3 parts, and the plots for each of them were then drawn as well. The 3 energy ranges were:

- $1 \mathrm{MeV}-100 \mathrm{MeV}$

- $100 \mathrm{MeV}-1 \mathrm{GeV}$

- $1 \mathrm{GeV}-2.4 \mathrm{GeV}$ 


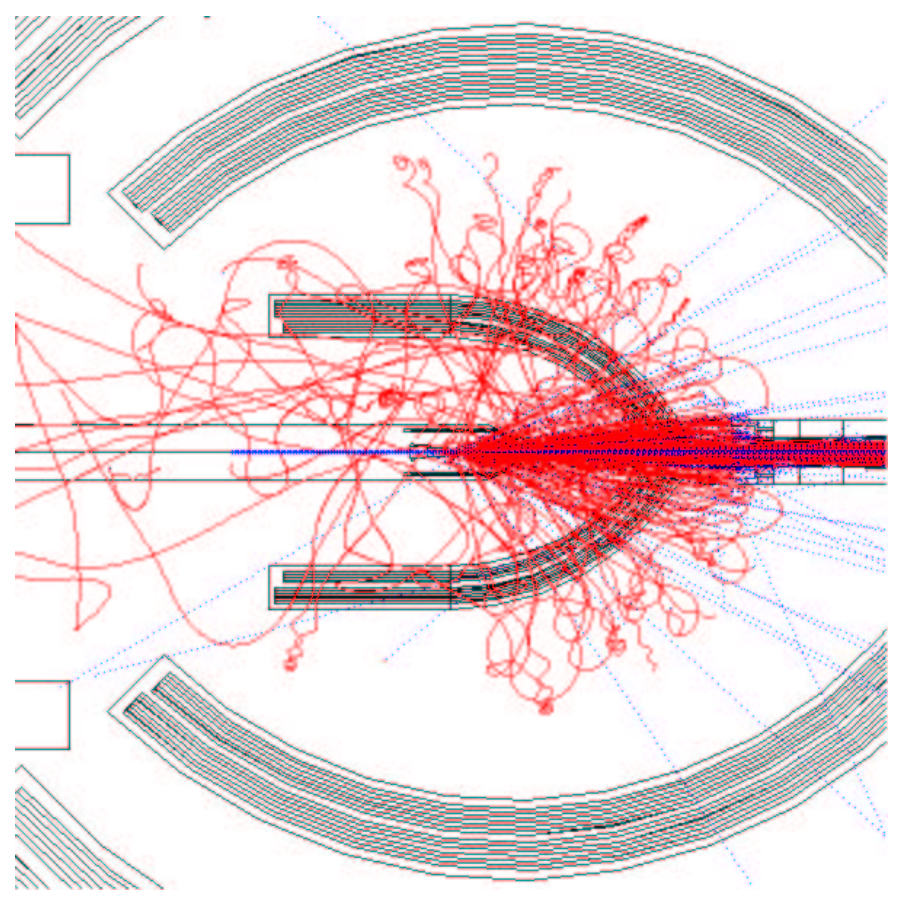

Figure 3.10: The accumulated low energy $e^{+} e^{-}$pair background for 1000 incoming bremstrahlung photons with energies between $1 \mathrm{MeV}$ and $2.4 \mathrm{GeV}$. Case with NO mini torus in.

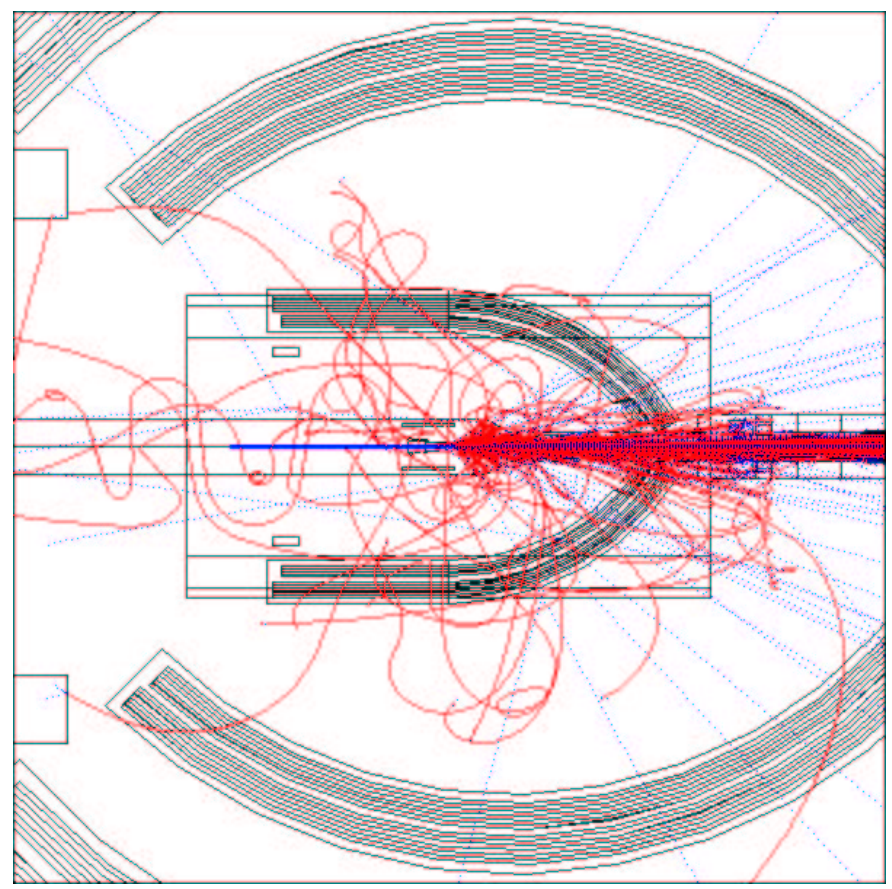

Figure 3.11: The accumulated low energy $e^{+} e^{-}$pair background for 1000 incoming bremstrahlung photons with energies between $1 \mathrm{MeV}$ and $2.4 \mathrm{GeV}$. Case with $75 \%$ of the maximum mini torus current. 


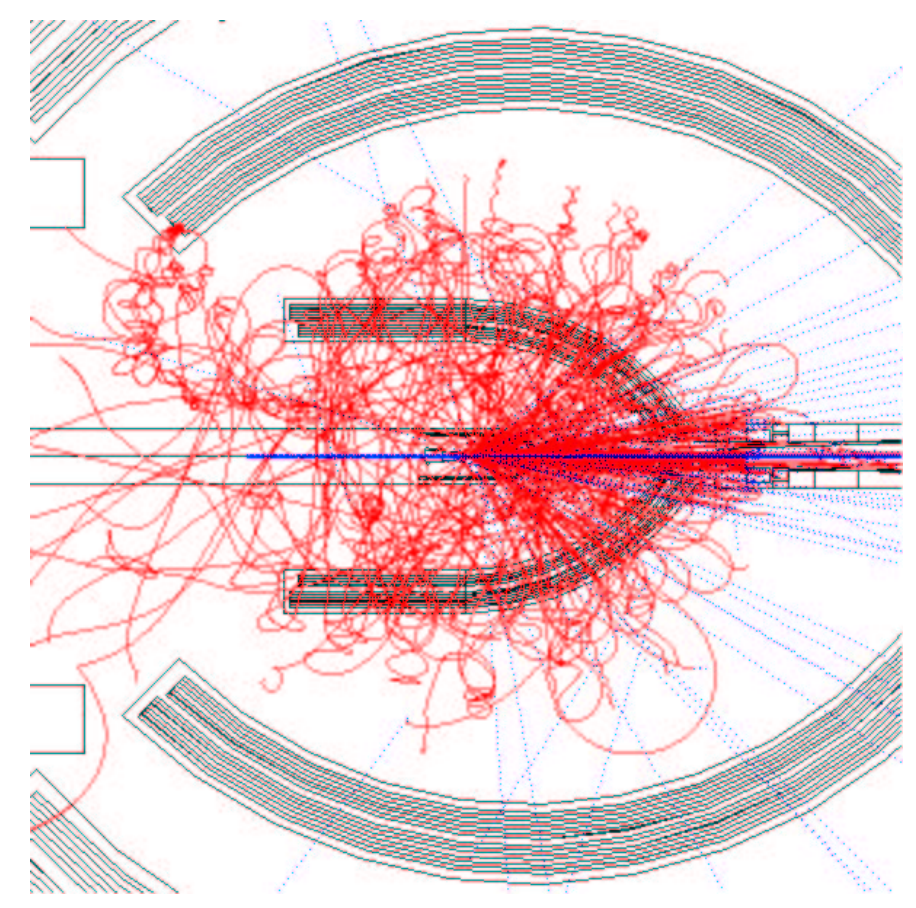

Figure 3.12: The accumulated low energy $e^{+} e^{-}$pair background for 1000 incoming bremstrahlung photons with energies between $1 \mathrm{MeV}$ and $100 \mathrm{MeV}$. Case with NO mini torus in.

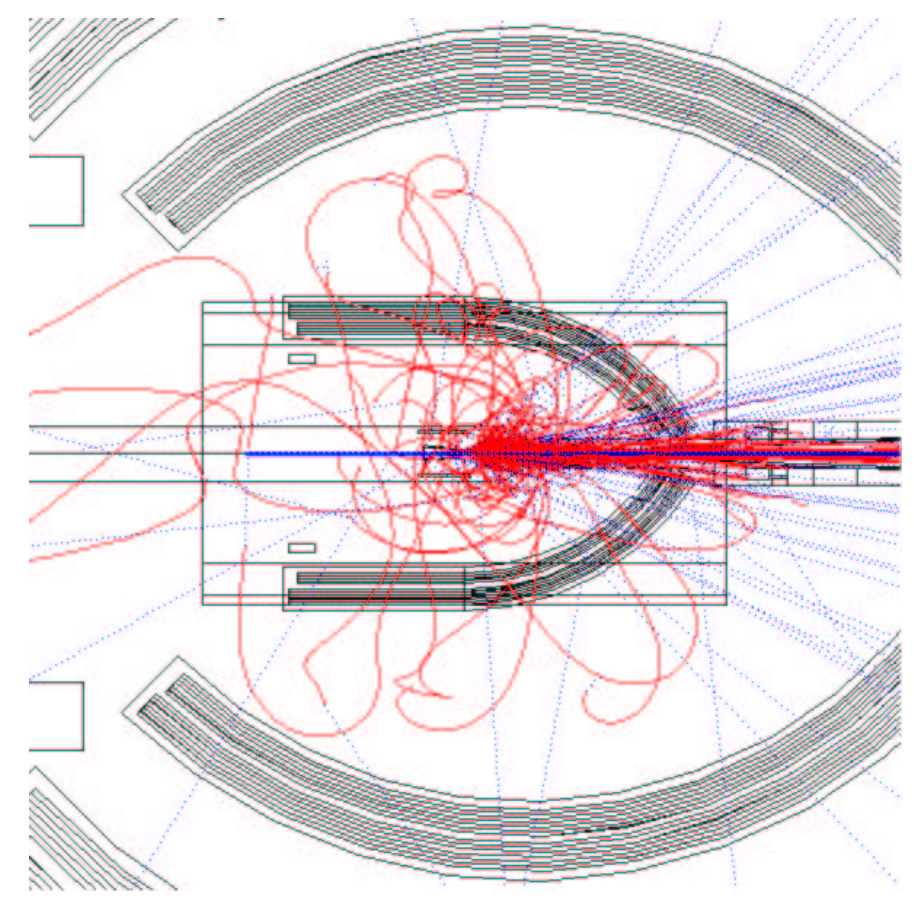

Figure 3.13: The accumulated low energy $e^{+} e^{-}$pair background for 1000 incoming bremstrahlung photons with energies between $1 \mathrm{MeV}$ and $100 \mathrm{MeV}$. Case with $75 \%$ of the maximum mini torus current. 


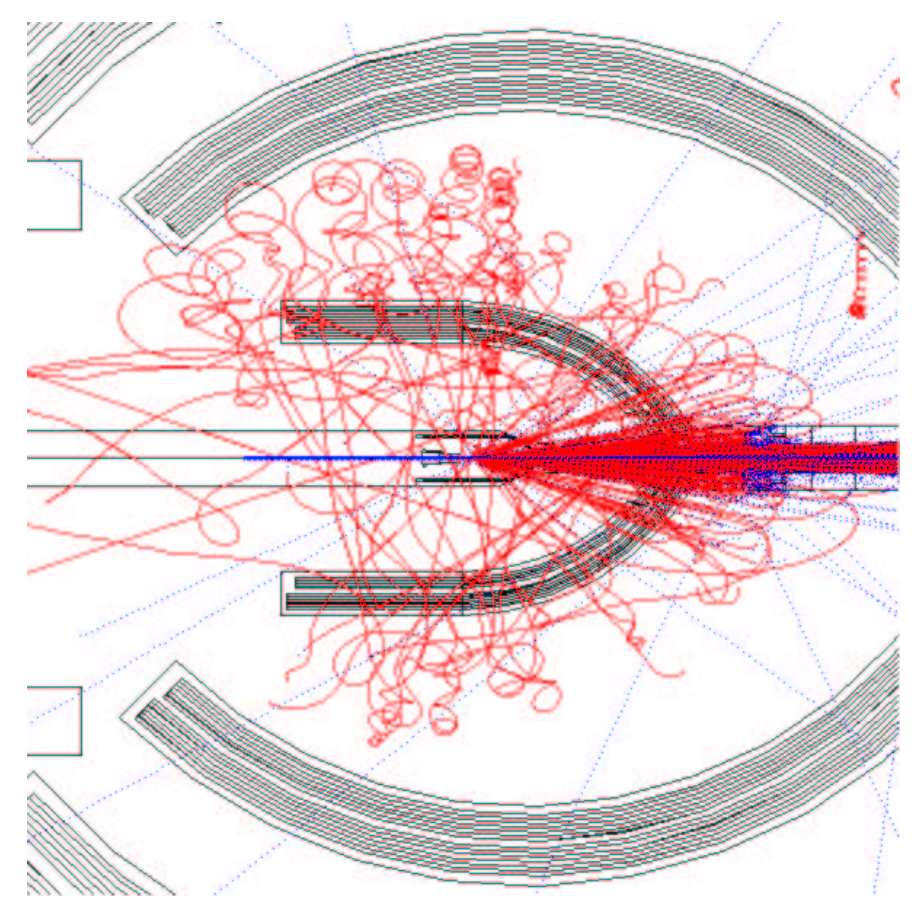

Figure 3.14: The accumulated low energy $e^{+} e^{-}$pair background for 1000 incoming bremstrahlung photons with energies between $100 \mathrm{MeV}$ and $1 \mathrm{GeV}$. Case with NO mini torus in.

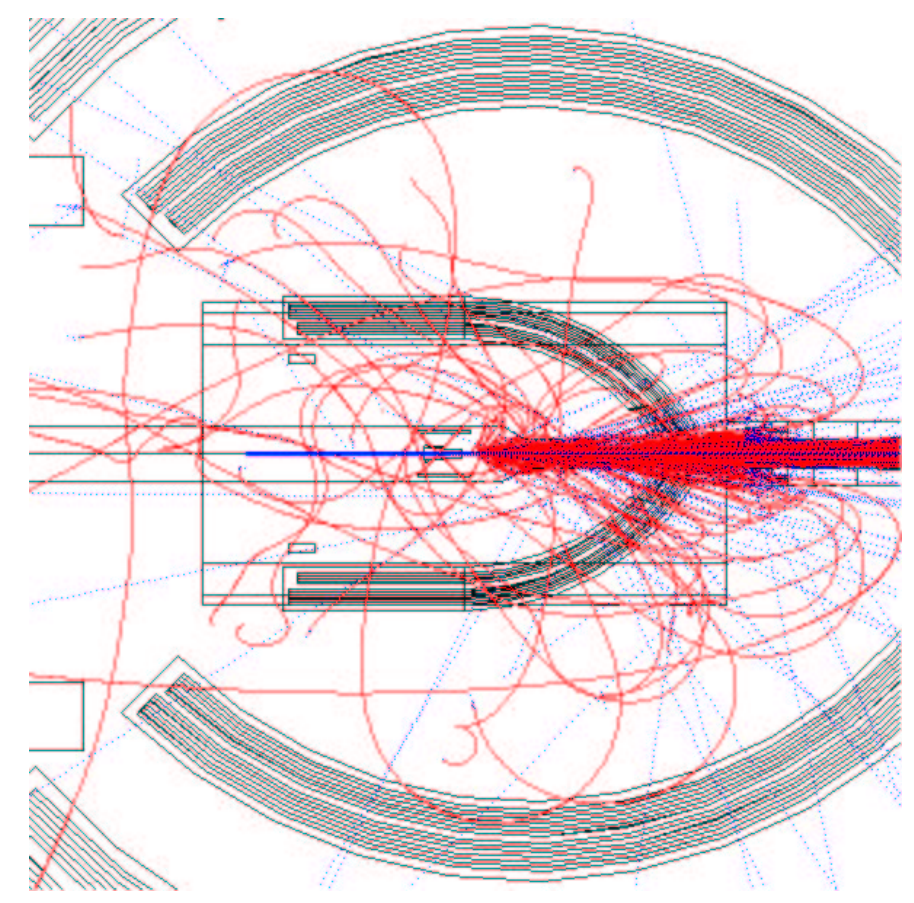

Figure 3.15: The accumulated low energy $e^{+} e^{-}$pair background for 1000 incoming bremstrahlung photons with energies between $100 \mathrm{MeV}$ and $1 \mathrm{GeV}$. Case with $75 \%$ of the maximum mini torus current. 


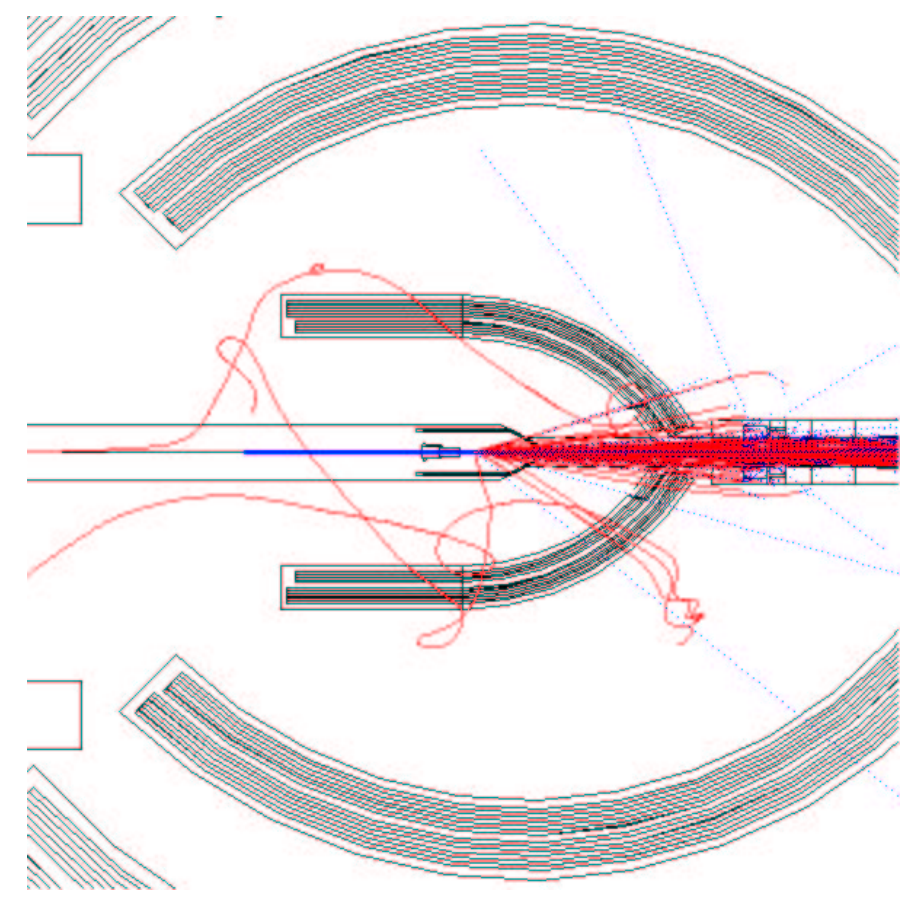

Figure 3.16: The accumulated low energy $e^{+} e^{-}$pair background for 1000 incoming bremstrahlung photons with energies between $1 \mathrm{GeV}$ and $2.4 \mathrm{GeV}$. Case with NO mini torus in.

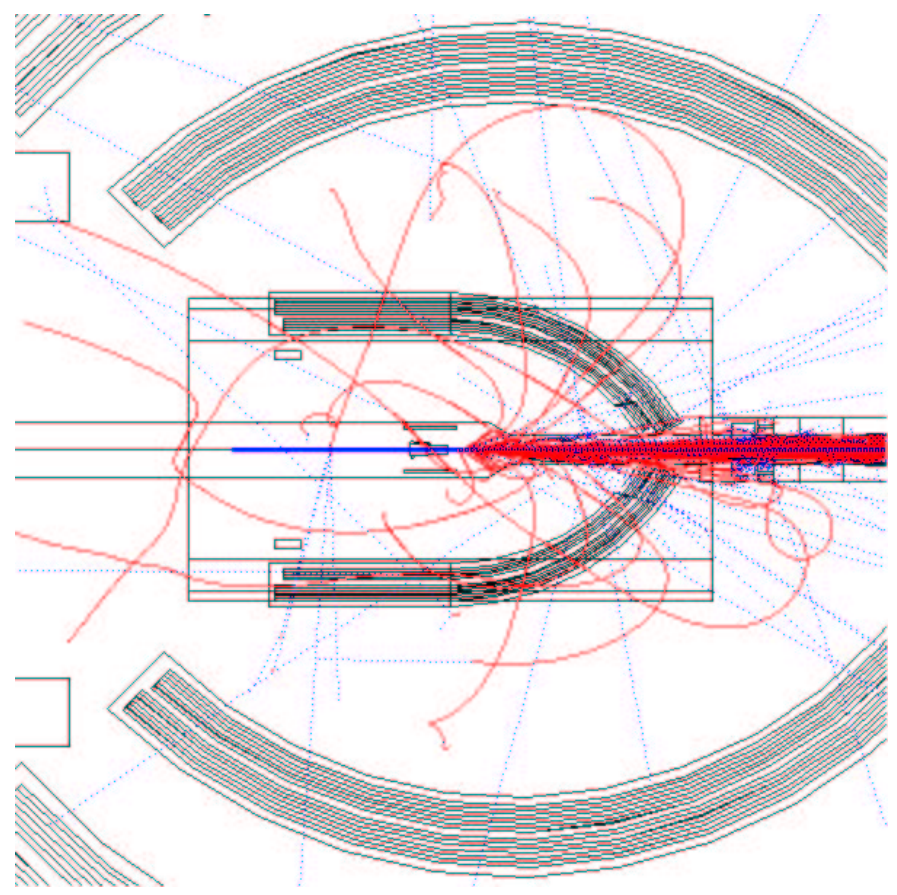

Figure 3.17: The accumulated low energy $e^{+} e^{-}$pair background for 1000 incoming bremstrahlung photons with energies between $1 \mathrm{GeV}$ and $2.4 \mathrm{GeV}$. Case with $75 \%$ of the maximum mini torus current. 


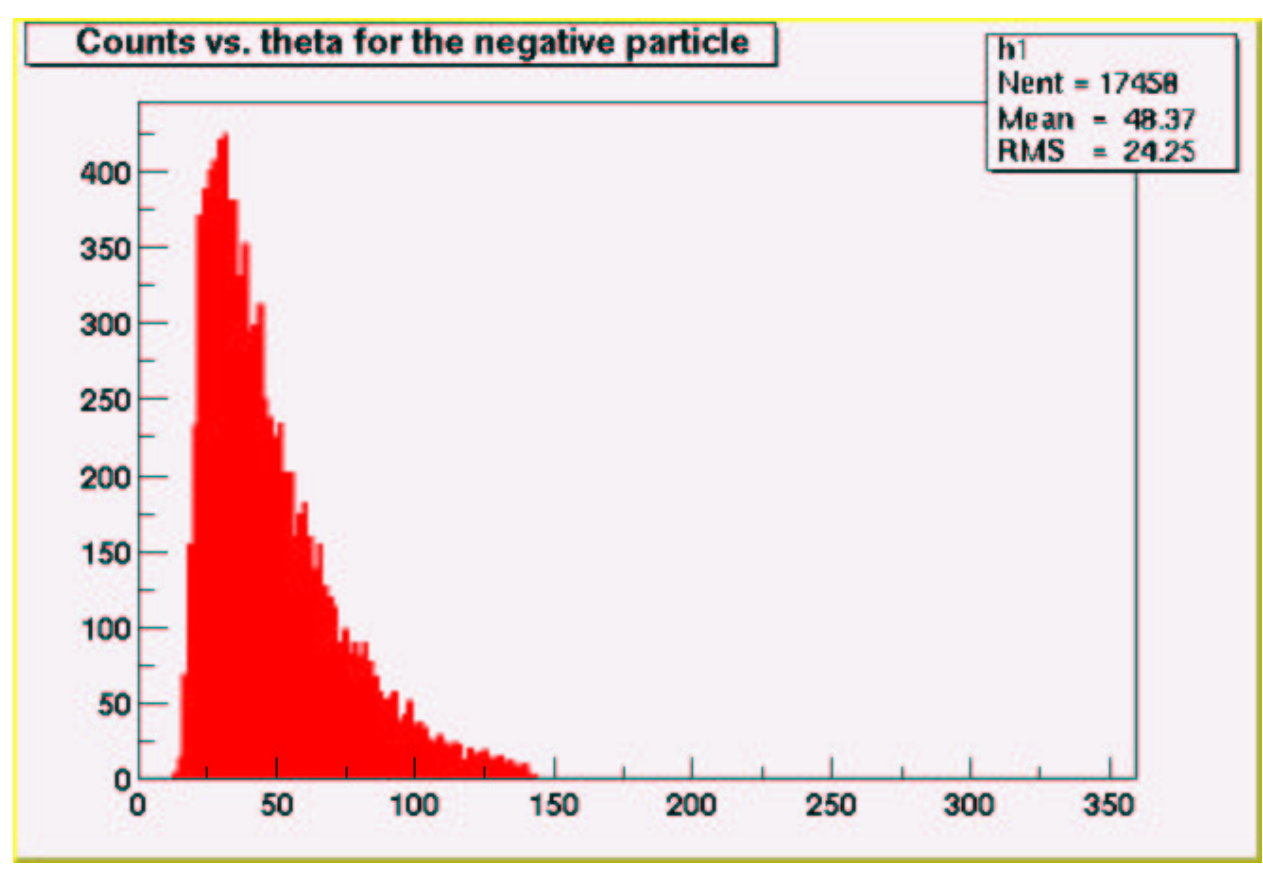

Figure 3.18: Number of $e^{-}$or $\pi^{-}$particles versus the scattering angle $\theta$ (in degrees).

One can see that a lot of the background is coming from the photons with energies in the range $1 \mathrm{MeV}-100 \mathrm{MeV}$. And this is also the range for which the mini torus field seems the most efficient. The mini torus seems to be bending all these low energy electrons and positrons into the beam pipe, so that the region I of the drift chambers does not see as many of them any more.

\subsection{Other issues}

\subsubsection{The angular distribution of the outgoing lepton pair}

For 50000 events of the type $\gamma p \rightarrow \rho p \rightarrow e^{+} e^{-} p$ or $\gamma p \rightarrow \rho p \rightarrow \pi^{+} \pi^{-} p$, the number of negative particles versus the scattering angle $\theta$ was plotted, and then the same was done for the positive particles and finally the number of $e^{+} e^{-}$ pairs versus the opening angle between them was plotted. This is illustrated in Figures 3.18 through 3.20 . 


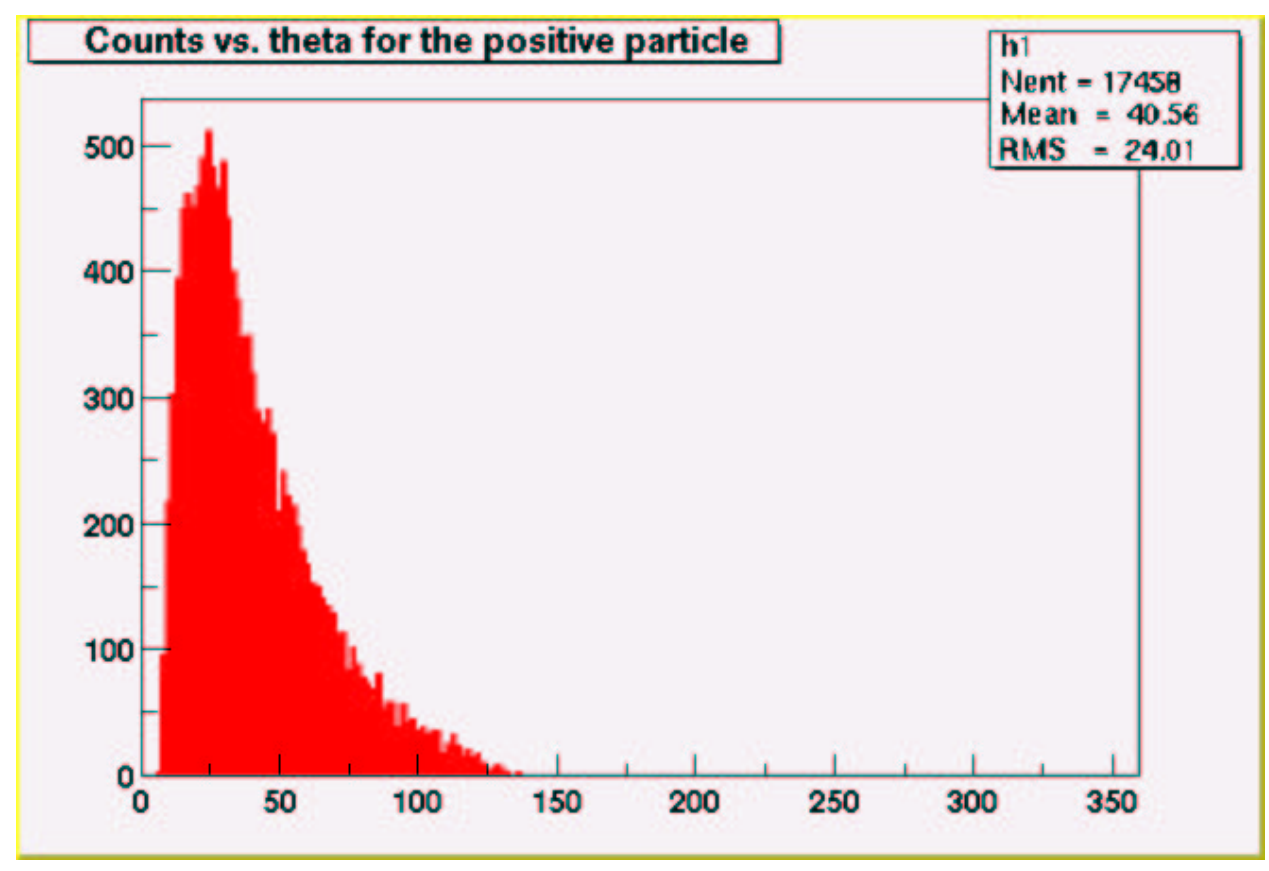

Figure 3.19: Number of $e^{+}$or $\pi^{+}$particles versus the scattering angle $\theta$ (in degrees).

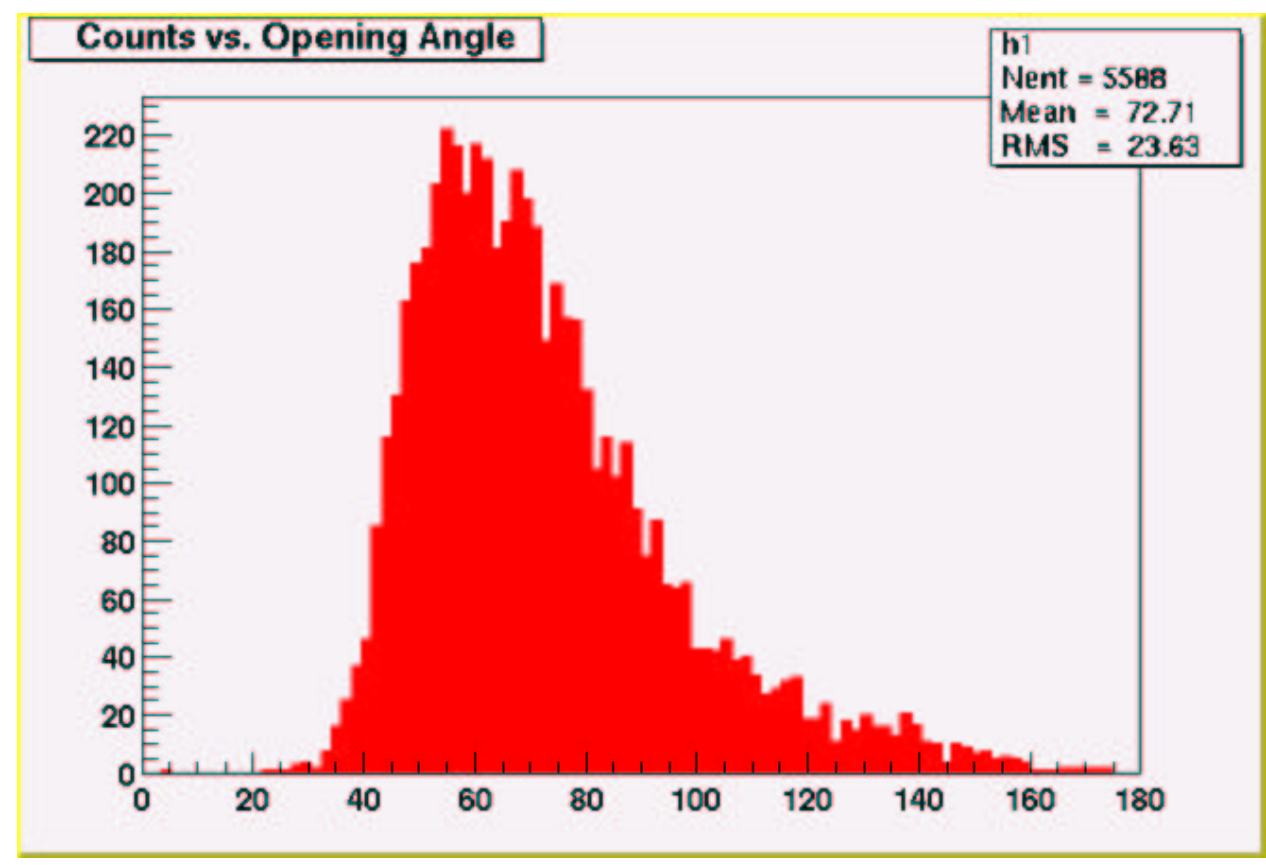

Figure 3.20: Counts versus the opening angle between the outgoing $e^{+} e^{-}$pairs (in degrees). 
The main concern by doing that, was to know if the main part of these particles were indeed going in the forward direction, since the Cerenkov counters and electromagnetic calorimeters, which are the two essential components in doing a good electron/pion discrimination, are essentially in the forward region of CLAS.

One can see from the plots presented that the bulk of these particles do indeed go towards the forward region of CLAS, were the Cerenkov counters and the electromagnetic calorimaters can detect them.

\subsubsection{The events that give 2 hits in the same sector of CLAS}

One of the other concerns before the run was to know what percentage of the outgoing $e^{+} e^{-}$pairs were both hitting the same sector of the detector. The way triggers are set in CLAS, it is impossible to trigger on this type of event, so they are expected to be lost. Now, what one may fear then, is that, this may distort the invariant mass spectra if these pairs that give hits in the same sector are coming from some particular region of the $\rho$ mass spectrum and if this effect is too large. In Figure 3.21, the counts versus the $e^{+} e^{-}$invariant mass squared for 41012 events of the type $\gamma p \rightarrow \rho p \rightarrow e^{+} e^{-} p$ are plotted, then the same was done to get Figure 3.22 requiring that only the pairs that give hits in the same sector of the detector be taken into account.

As one may see only 2725 of such events appeared to be there, that is about $6.6 \%$ of the initial number and they seem to be preferentially coming from the low end tail of the $\rho$. The conclusion was that this is a small enough fraction as not to fear any significant distortion due to the possible loss of those events. 


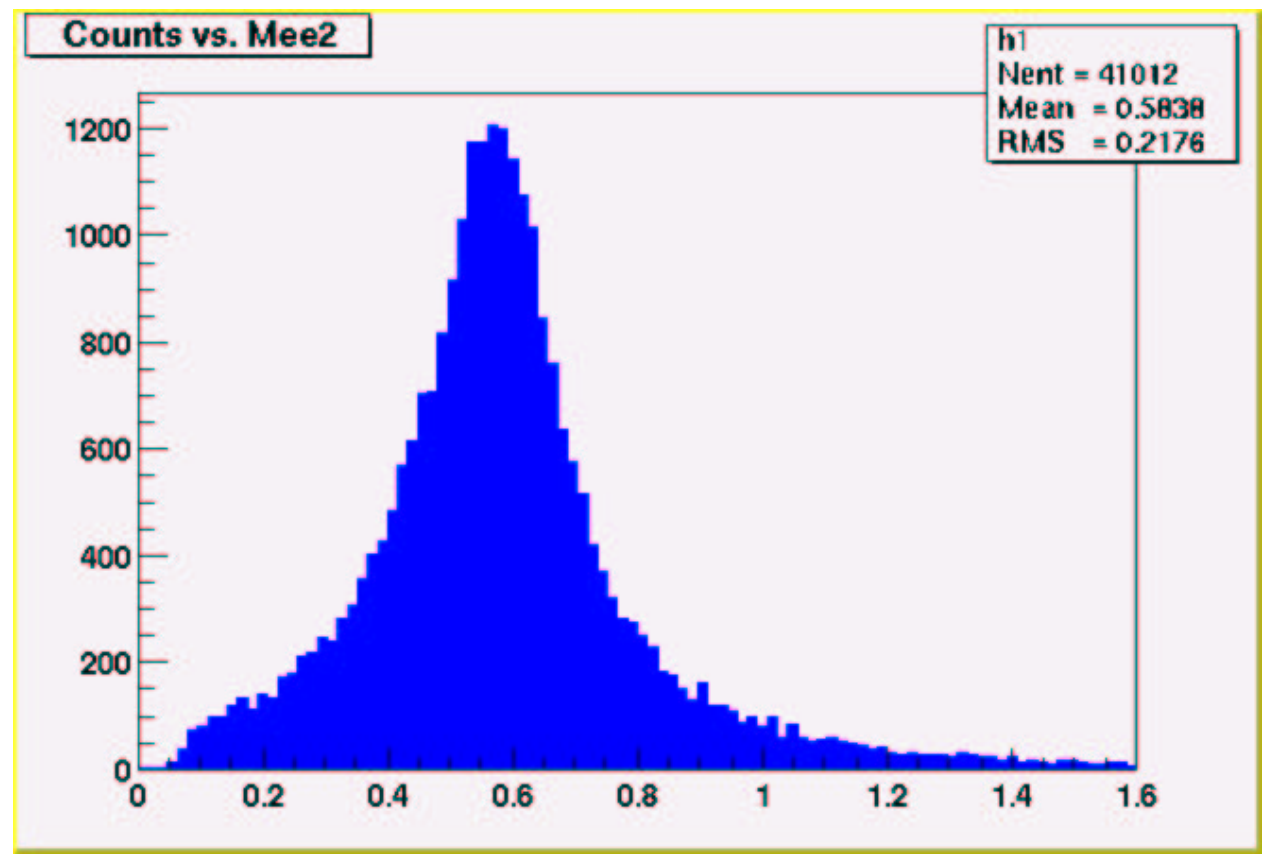

Figure 3.21: Counts versus the $e^{+} e^{-}$invariant mass squared (in $\mathrm{GeV}^{2}$ ) for events of the type $\gamma p \rightarrow \rho p \rightarrow e^{+} e^{-} p$.

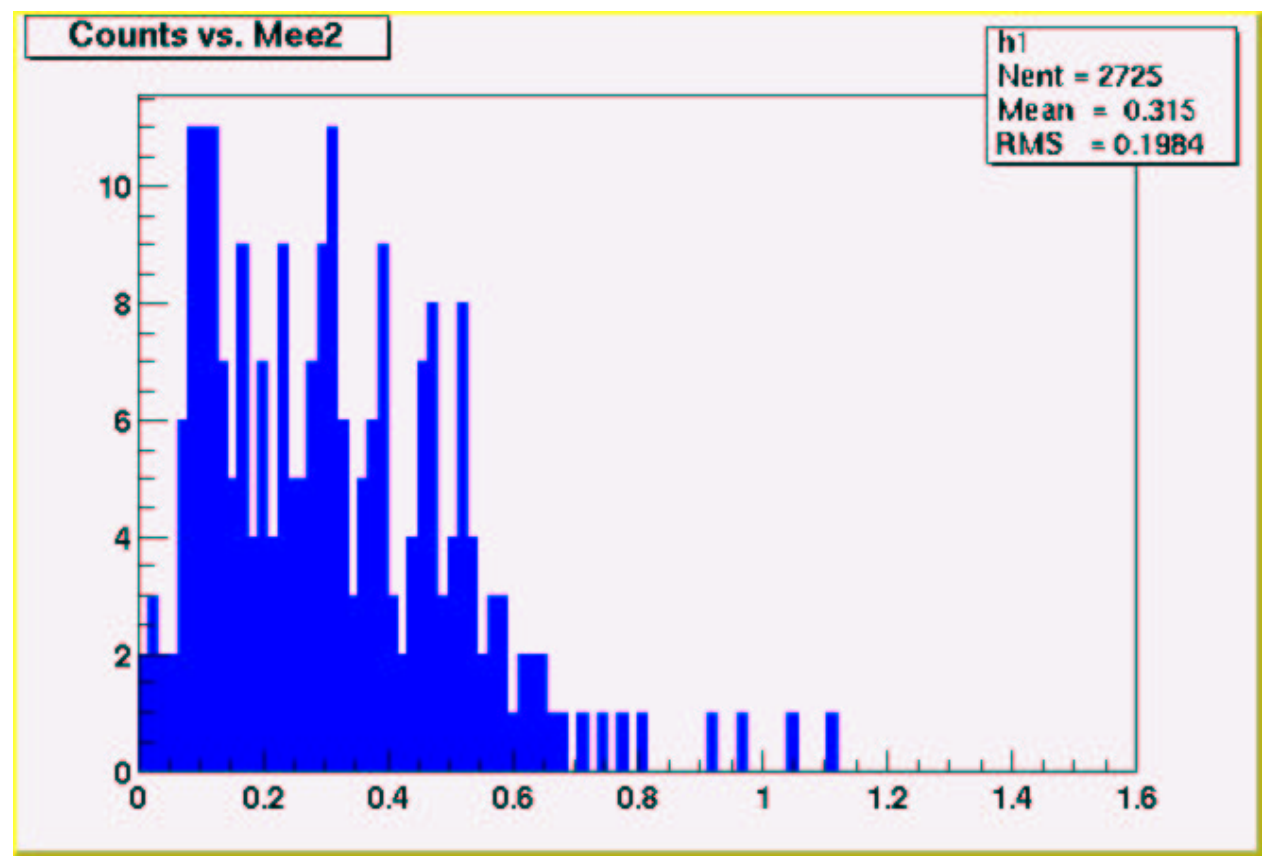

Figure 3.22: Counts versus invariant mass squared (in $\mathrm{GeV}^{2}$ ) of those $e^{+} e^{-}$pairs that give hits in the same sector of CLAS, for events of the type $\gamma p \rightarrow \rho p \rightarrow$ $e^{+} e^{-} p$. 


\subsubsection{The electron/pion discrimination method}

This work was done by M. Kossov prior to the 2001 proposal of $\mathrm{g} 7$ and is explained thoroughly in the reference [Kos01]. A summary of the e/ $\pi$ discrimination algorithm proposed there will be given in the following paragraphs, since being able to separate electrons from pions is one of the major challenges for the analysis of the g7 data.

The method was tested on electron data (e1c experiment) since the timing for g7 will be closer to the electron run methods than the normal photon run methods (the photon runs usually have a scintillator called the "start counter" around the target to get the vertex time, while electron runs do not have it since they need to have the mini torus in and one can not have them both due to the fact they occupy the same physical space).

The first step of the discrimination method is to select events with a good timing, or otherwise stated to select events with a clearly identified scattered electron, and then identify the correct RF bucket. Then, two velocities are computed for each particle using the electron mass or the pion mass according to the formula:

$$
\beta=\frac{p}{\sqrt{p^{2}+m_{\pi / e}^{2}}}
$$

The time of the interaction is determined by the RF time. It is also calculated for each particle from its time of flight given by the CLAS TOF scintillators. The first cut, called the "silver cut", is such that the difference between these two times is less than $1 \mathrm{~ns}$ and is applied to the two relativistic particles. A similar, but tighter cut is applied to the scattered electron.

After this, the particles with a momentum below $0.2 \mathrm{GeV}$ have a large enough difference in $\beta$ to be discriminated. However the electrons are of no interest to g7 since they increase the low effective mass background without increasing the acceptance. So, a momentum cut is applied then, that suppresses all of these events. 
Then comes the second cut, called the "golden cut" that uses both the Cerenkov counter (CC) and the electromagnetic calorimeter (EC). The electrons develop a shower in the EC as soon as they enter it and so lose most of their energy in its inner layers, while the pions deposit very little energy throughout the EC. The requirements of the golden cut use this knowledge and set the total energy deposited in the EC to greater than $0.2 \mathrm{GeV}$, an energy in the inner part of the EC to greater than $0.05 \mathrm{GeV}$ and demand a corresponding hit in the CC.

The final cut, called the "platinium cut", is of kinematical nature. To help suppress the random coincidences and misidentified events, this cut requires that the missing mass of the scattered electron plus the two relativistic charged particles matches the mass of the proton i.e. $0.78 \mathrm{GeV}^{2}<m m_{e r^{+} r^{-}}<1 \mathrm{GeV}^{2}$. In addition it demands that the energy conservation factor $\left(\triangle^{2}=\left(E_{\text {in }}^{e}+M_{p}-E_{\text {out }}^{e}-\right.\right.$ $\left.\left.E_{\text {out }}^{r^{+}}-E_{\text {out }}^{r^{-}}\right)^{2}+\left(\vec{p}_{\text {in }}^{e}-\vec{p}_{\text {out }}^{p}-\vec{p}_{\text {out }}^{e}-\vec{p}_{\text {out }}^{r^{+}}-\vec{p}_{\text {out }}^{r^{-}}\right)^{2}\right)$ be less than $0.02 G e V^{2}$. The final step uses a quality factor of the CC quantifying the quality of the match between the track and the corresponding hit in the CC.

Using these cuts, the author of reference [Kos01] was able to get a rejection factor better than $5 \times 10^{-6}$ for the e1c data. The method still needs to be tested on the g7 data during its analysis and further improved if necessary. 


\section{Chapter 4}

\section{The g7 test run}

This test run started at 11:30 pm. June 14, 2002. It took about 12 hours to complete, and so ended at noon June 15, 2002. The main goal of the test was to check the accuracy of the background simulations and see if indeed the mini torus helped reduce the low energy background electron and positron pairs that seemed to flood the region I of the drift chambers according to the simulations, and if so, what reduction that exactly amounted to.

The test run took place in the middle of the e $2 \mathrm{~b}$ experiment. The g7 lead foil was placed in the beam line and bremstrahlung photons were sent on it, produced by an initial electron beam of energy $4.7 \mathrm{GeV}$, at first at a current of $20 \mathrm{nA}$. Data was taken in the case of $0 \%, 25 \%, 50 \%$, and $75 \%$ of the maximum mini torus current. The number of hits in the drift chambers per region and sector was recorded. In the case of $75 \%$ of the maximum mini torus field, about 3 times less hits were seen in the region I of the drift chambers, compared to the case with $0 \%$ of mini torus current, thus giving an excellent agreement with the simulations. Then, the lead foil was taken off and replaced a carbon foil and the same procedure was repeated with the carbon foil. The results for the carbon 
were comparable to the ones for the lead.

Also, the beam current was increased until it made the drift chambers trip, to test our limitations for the actual run, with the lead foil in. It was not until a $60 \mathrm{nA}$ current was reached, that the sectors 1 and 4 of the region III of the drift chambers tripped. A possible explanation for this is, the extra hits in the mentioned sectors of the region III that made it trip, might have been background coming from the tagger dump or from the scattering of the beam particles on the collimator. So it was essentially due to shielding problems. Extra shielding was used during the actual run based on these conclusions. 


\section{Chapter 5}

\section{Summary and conclusion}

The g7 experiment has been devised to measure the modifications of the vector meson properties, such as mass or width, inside nuclear medium, based on the ideas presented in the numerous papers published during the fifteen years that preceded its run. It consisted in sending a bremstrahlung photon beam on a target that contained elements with different densities, a liquid deuterium cell, and seven solid foils: carbon, iron, carbon, lead, carbon, titanium, carbon. The goal of the experiment is to examine the inclusive $e^{+} e^{-}$photoproduction in the incoherent region. The reaction of interest to $\mathrm{g} 7$ is

$$
\gamma A \rightarrow V A^{\prime} \rightarrow e^{+} e^{-} A^{\prime}
$$

where $\mathrm{V}$ could be a $\rho$ an $\omega$ or a $\phi$ meson.

The goal of the present thesis was to present the simulation work done prior to the $\mathrm{g} 7$ run in the fall of 2002 at Jlab, essential for the choice of the ideal experimental setup and conditions, as well as the test run of June 2002. The simulations needed a particular attention, given the many experimental challenges that awaited the g7 team. First, one had to prove that the resolution of the CLAS detector was sufficient to properly locate the vertices of the events 
given the multi-segmented target, and the simulations proved that fact. They also provided a rough idea of the systematic errors that one had to expect. Using nuclei bigger than carbon was a first time for CLAS. Given the very small branching ratio for $\rho \rightarrow e^{+} e^{-}$, a very intense beam had to be sent on the target containing high-Z material. Thus, a huge background, formed of low energy $e^{+} e^{-}$ pairs, was expected around the target and the region I of the drift chambers and which one had to reduce in an efficient way. The simulations showed that using the mini torus with its current set to $75 \%$ of its maximum value would give a reduction of about a factor of 3 in the number of hits in the region I of the drift chambers, compared to the case with no mini torus in, which the test run confirmed. In addition, the branching ratio for $\rho \rightarrow \pi^{+} \pi^{-}$being five orders of magnitude bigger than the one for $\rho \rightarrow e^{+} e^{-}$, a very rigorous way of discriminating the electrons from the pions had to be devised, where the g7 team had again to take up the challenge for the first time in the collaboration. A method of electron/pion discrimination with CLAS has been briefly presented and still needs to be tested on the g7 data.

The simulations, the test run, and hours of brainstorming, not forgetting the always precious help and advice of the CLAS collaborators, have been at the base of the successful g7 run in the fall of 2002. The analysis of its data and the hopefully enlightening results will be presented in my $\mathrm{PhD}$ thesis. 


\section{Appendix A}

\section{The simulation tools}

\section{The simulation package}

A package that is commonly used by the Hall-B collaborators, called GSIM, was used to do the simulations. This package makes use of GEANT to simulate the CLAS detector. The only addition to the package was a file containing the description of the g7 target. The package also contains files that describe other experiment targets. The choice of the target that one wants to use for a given set of simulations, as well as the choice of some other parameters, such as kinematical cuts or the value of the main torus or mini torus current, can be specified in a file called ffread.in, that is given as an option in the command line. It has a batch version and an interactive version that enables one to see what is really happening in the detector. More details on the package and how it needs to be used can be found on the Hall-B web page (www.jlab.org/Hall-B) by clicking on the GSIM link.

\section{The event generators}

Three event generators were used to generate the events depending on the situation. The generator called he3 (by P. Corvisiero and L. Mazzaschi in 1992) 
can create interactions between a photon and a proton or a helium 3 nucleus. It was used to generate events of the type $\gamma p \rightarrow \rho p \rightarrow e^{+} e^{-} p$. It is possible to have other mesons than the $\rho$ and also to have several channels simultaneously. The generator called ppgen (by D. P. Weygand) was used to generate events of the type $\gamma p \rightarrow e^{+} e^{-} p$ (he3 was the one used preferentially). Finally, to simply generate a bremstrahlung photon beam distibuted in a disc of $\mathrm{xxx} \mathrm{cm}$ diameter in the $\mathrm{x}-\mathrm{y}$ plane and with momentum parallel to the z-axis, a generator called gen_beam (by D. J. Tedeschi) was used.

\section{The reconstruction program}

A program called a1c, that the photon run experimenters of Hall-B all use commonly to reconstruct the events (or "cook" them, in the jargon of the nuclear physicists), was used. It comes with a -h flag that gives a list of all the other flags that one can put on the command line as option to reconstruct the events the way one chooses to recontruct them. 


\section{REFERENCES}

[Ada01] G. Adams et al., Nucl. Instr. Methods A 465, 414 (2001).

[Aga95] G. Agakichiev et al., Phys. Rev. Lett. 75, 1272 (1995).

[ali] http://alice.web.cern.ch/Alice/.

[Ama01] M. Amarian et al., Nucl. Instr. Methods A 460, 239 (2001).

[Asa93] M. Asakawa and C. M. Ko, Phys. Rev. C 48, R526 (1993).

[Bau78] T. H. Bauer, R. D. Spital, and D. R. Yennie, Reviews of Modern Phys. 50, 261 (1978).

[Ber90] P. Y. Bertin and P. A. Guichon, Phys. Rev. C 42, 1133 (1990).

[Ber94] P. Y. Bertin, M. Kossov, and B. M. Preedom, 1994, CEBAF Proposal PR94-002.

[Bro88] G. E. Brown, Nucl. Phys. A 488, 659c (1988).

[Bro91] G. E. Brown and M. Rho, Phys. Rev. Lett. 66, 2720 (1991).

[Bro00] W. Brooks, Nucl. Phy. A 663, 1077c (2000).

[Cas95] W. Cassing, W. Ehehalt, and C. M. Ko, Phys. Lett. B 363, 35 (1995).

[Cas98] W. Cassing, E. L. Bratkovskaya, R. Rapp, and J. Wambach, Phys. Rev. C 57, 916 (1998).

[cer] http://www.weizmann.ac.il/ cherlin/ceres.html.

[Cha96] G. Chanfray, R. Rapp, and J. Wambach, Phys. Rev. Lett. 76, 368 (1996).

[Dja01] C. Djalali, D. P. Weygand, and M. Kossov, 2001, CEBAF Proposal PR01-112. 
[Eff99] M. Effenberger, E. L. Bratkovskaya, and U. Mosel, Phys. Rev. C. 60, 044614 (1999).

[Eff00] M. Effenberger and U. Mosel, Phys. Rev. C. 62, 014605 (2000).

[Ele97] V. L. Eletsky and B. L. Ioffe, Phys. Rev. Lett. 78, 1010 (1997).

[En'00] H. En'yo et al., Nucl. Phys. A 670, 182c (2000).

[Gil02] J. Gillies, 2002, Probing the boundary of nuclear-particle physics, CERN Courier, May.

[Gui89] P. A. M. Guichon, Nucl. Phys. A 497, 265c (1989).

[had] http://www-hades.gsi.de/gsi/.

[Hag02] K. Hagiwara et al., 2002, Review of Particle Physics.

[Hat91] T. Hatsuda and S. H. Lee, Phys. Rev. C 46, R34 (1991).

[Kag99] M. A. Kagarlis et al., Phys. Rev. C 60, 025203 (1999).

[Koc96] V. Koch and C. Song, Phys. Rev. C 54, 1903 (1996).

[Kon98] L. A. Kondratyuk, A. Sibirtsev, W. Cassing, Y. S. Golubeva, and M. Effenberger, Phys. Rev. C 58, 1078 (1998).

[Kos01] M. Kossov, 2001, CLAS-NOTE-2001-009: e/pi Rejection in CLAS.

[Lee01] C. W. Leemann, D. R. Douglas, and G. A. Krafft, Annu. Rev. Nucl. Part. Sci. 51, 413 (2001).

[Li95] G. Q. Li, C. M. Ko, and G. E. Brown, Phys. Rev. Lett. 75, 4007 (1995).

[lol] http://www.phys.uregina.ca/sparro/gjlolos/index.html.

[Lol98] G. J. Lolos et al., Phys. Rev. Lett. 80, 241 (1998).

[Mas95] M. Massera et al., Nucl. Phys. A 590, 93c (1995).

[Mec02] B. A. Mecking et al., Oct. 2002, The CEBAF Large Acceptance Spectrometer (CLAS), preprint submitted to Elsevier Science.

[Mes00] M. D. Mestayer et al., Nucl. Instr. Methods A 449, 81 (2000).

[Oza01] K. Ozawa et al., Phys. Rev. Lett. 86, 5019 (2001). 
[Oza02] K. Ozawa et al., Nucl. Phys. A 698, 535c (2002).

[rhi] http://www.bnl.gov/RHIC/.

[Ros96] P. Rossi et al., Nucl. Instr. Methods A 381, 32 (1996).

[Sha93] C. M. Shakin and W.-D. Sun, Phys. Rev. C 49, 1185 (1993).

[Smi99] E. S. Smith et al., Nucl. Instr. Methods A 432, 265 (1999).

[Sob00] D. I. Sober et al., Nucl. Instrum. Methods A 440, 263 (2000).

[Son96] C. Song, V. Koch, S. H. Li, and C. M. Ko, Phys. Lett. B 52, 379 (1996). 